University of Louisville ThinkIR: The University of Louisville's Institutional Repository

Electronic Theses and Dissertations

$12-2015$

\title{
Interdisciplinary palliative care teams : insights and experiences of chaplain interns and social work interns.
}

Ann Hayes Ronald

University of Louisville

Follow this and additional works at: https://ir.library.louisville.edu/etd

Part of the Counseling Commons

\section{Recommended Citation}

Ronald, Ann Hayes, "Interdisciplinary palliative care teams : insights and experiences of chaplain interns and social work interns." (2015). Electronic Theses and Dissertations. Paper 2309.

https://doi.org/10.18297/etd/2309

This Doctoral Dissertation is brought to you for free and open access by ThinkIR: The University of Louisville's Institutional Repository. It has been accepted for inclusion in Electronic Theses and Dissertations by an authorized administrator of ThinkIR: The University of Louisville's Institutional Repository. This title appears here courtesy of the author, who has retained all other copyrights. For more information, please contact thinkir@louisville.edu. 


\title{
INTERDISCIPLINARY PALLIATIVE CARE TEAMS: INSIGHTS AND EXPERIENCES OF CHAPLAIN INTERNS AND SOCIAL WORK INTERNS
}

\author{
By \\ Ann Hayes Ronald \\ B. A., Hanover College, 1978 \\ M. Ed., University of Louisville, 2005

\begin{abstract}
A Dissertation
Submitted to the Faculty of the College of Education and Human Development

In Partial Fulfillment of the Requirements for the Degree of
\end{abstract}

Doctor of Philosophy in Counseling and Personnel Services

\author{
Department of Counseling and Human Development \\ University of Louisville \\ Louisville, Kentucky
}

December 2015 
Copyright 2015 by Ann Hayes Ronald

All rights reserved 

INTERDISICIPLINARY PALLIATIVE CARE TEAMS: INSIGHTS AND EXPERIENCES OF CHAPLAIN INTERNS AND SOCIAL WORK INTERNS

\author{
By \\ Ann Hayes Ronald \\ B. A., Hanover College, 1978 \\ M. Ed., University of Louisville, 2005
}

A Dissertation Approved on

November 20, 2015

By the following Dissertation Committee:

Dissertation Director, Lisa M. Hooper, Ph.D.

Eileen O. Estes, Ph.D.

Melissa Evans-Andris, Ph.D.

Barbara A. Head, Ph.D. 


\section{DEDICATION}

I would like to dedicate this work to my husband, Peter, and my daughters, Mollie and Sarah. Graduate school has been a long journey and one I could not have completed without your unending support. Thank you. I love you all very much. 


\section{ACKNOWLEDGEMENTS}

I want to acknowledge:

Dr. Hooper, my chair whose energy revived my dissertation. Dr. Hooper provided excellent and timely feedback, helped me regain my confidence when it was lagging, and gave me support when I needed it.

Dr. Head, who provided me with learning environments for palliative care and an incredible source of data,

Dr. Estes, who taught the class that ignited my desire for further study, and

Dr. Evans-Andris, who showed me how to do qualitative research and analysis.

Her enthusiasm kept me on track.

I also want to thank:

Dr. Mark Pfeifer, for his interdisciplinary palliative care team study data and Amy Martin, R.N., the research nurse who retrieved it for me.

I also want to acknowledge:

Dr. Nancy Cunningham and Dr. Patrick Hardesty, who were with me from the beginning and suggested I follow a qualitative research path.

Drs. Joseph Petrosko, Namock Choi, and Sam Stringfield, whose help and guidance in quantitative statistics helped me reach my goal.

Lastly, to Jennifer Dessert, my editor, for all her work.

Each of you helped me get here. I couldn't have done it without you. I thank each of you from the bottom of my heart. 


\section{ABSTRACT \\ INTERDISCIPLINARY PALLLIATIVE CARE TEAMS: INSIGHTS AND EXPERIENCES OF CHAPLAIN INTERNS AND SOCIAL WORK INTERNS}

\section{Ann Hayes Ronald}

November 20, 2015

In 2030, the population of those 65 years and older is projected to be 72 million, which will represent $20 \%$ of adults in the United States. This large group will have affected many aspects of our society, including families and healthcare providers. Decisions about medical care, caregiving, financial concerns, and new circumstances in living arrangements will be made. Perhaps no aspect of life will be as important as how one spends the end-of-life. Importantly, the care that individuals receive is likely an important aspect of this end-of-life experience.

Interdisciplinary palliative care teams provide effective and comprehensive endof-life care for patients and their families; however, barriers exist that often impede their effectiveness. The current qualitative study explored some of the often-reported barriers evidenced in the literature base; more specifically, the study explored written reflections of chaplain and social work interns after they completed clinical placement with an interdisciplinary palliative care team. Findings from the data revealed five themes (i.e., Learning Lessons, Disappointment, Being There, Dealing with Family Matters, and Making Hard Decisions), which aligned with both groups of interns. Two themes were identified that describe unique chaplain or social work discipline-specific roles and interventions (i.e., Intern as Existential Comforter and Intern as Psychosocial Resourcer). 
The meaning and importance of the findings in the context of the literature base are discussed. Directions for future research for palliative care and interdisciplinary teams are offered. Suggestions for diverse providers, counselors, and counselor training programs are provided. 
TABLE OF CONTENTS

PAGE

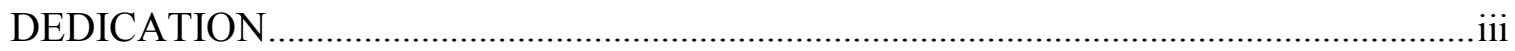

ACKNOWLEDGEMENTS .................................................................................................

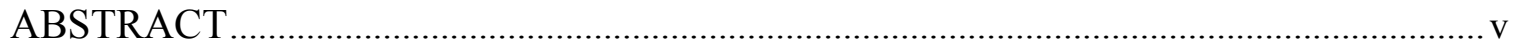

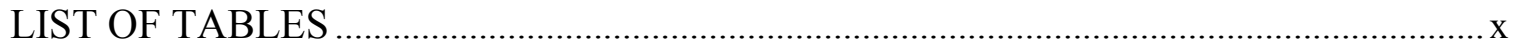

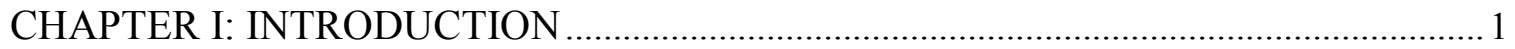

Conceptual Framework ……………………………...................................... 7

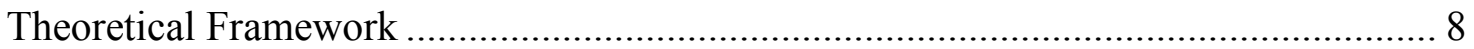

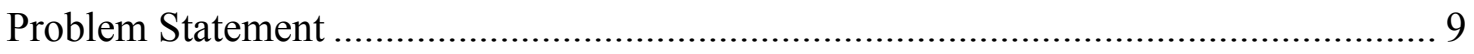

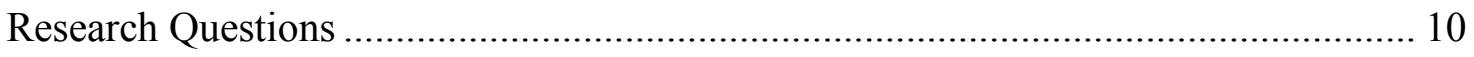

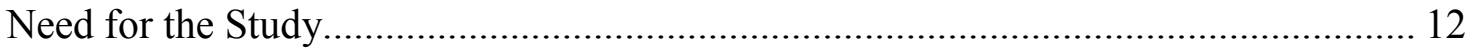

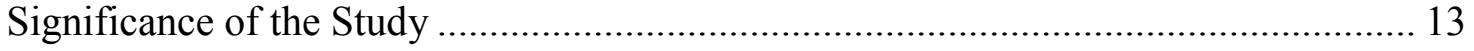

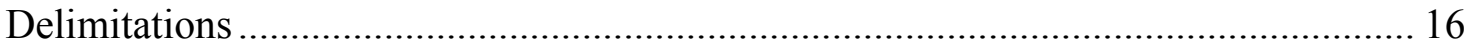

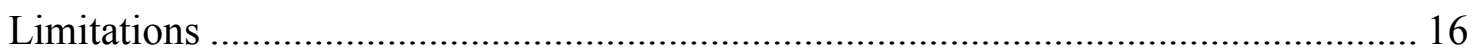

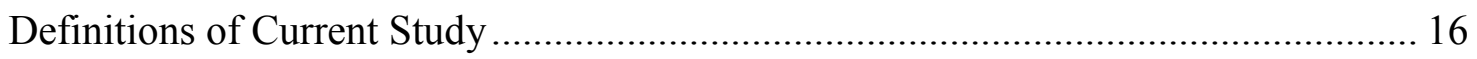

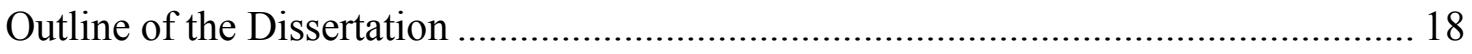

CHAPTER II: LITERATURE REVIEW ………………………………………........ 20

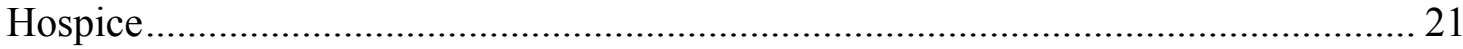

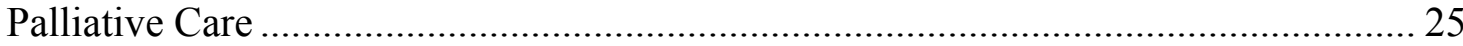

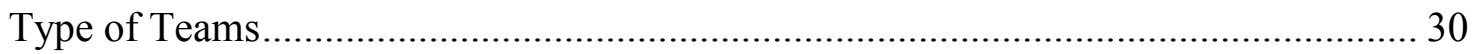

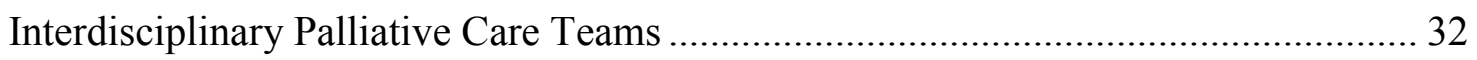

Interdisciplinary Palliative Care Team: Core Domains and Disciplines........................ 59

Existential Theory and Palliative Care.................................................................... 71

Summary and Discussion................................................................................... 76

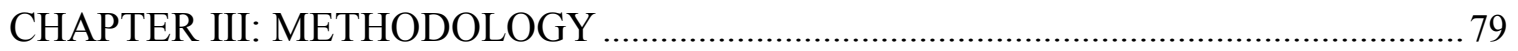

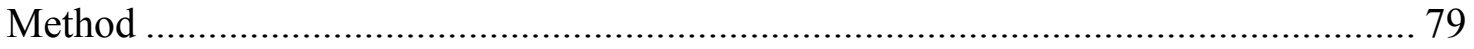

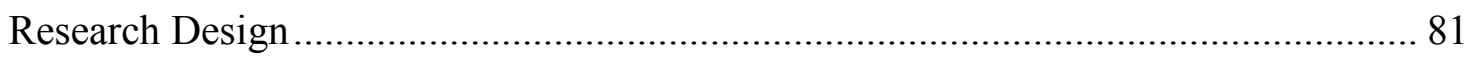

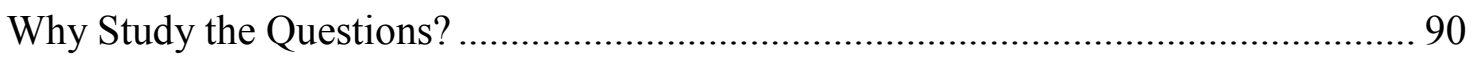

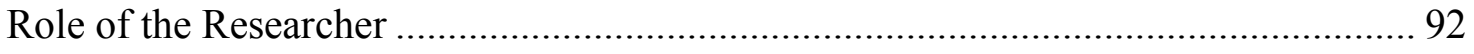




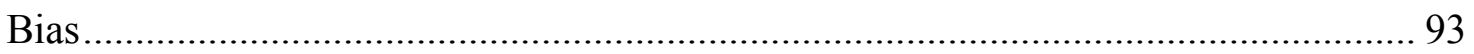

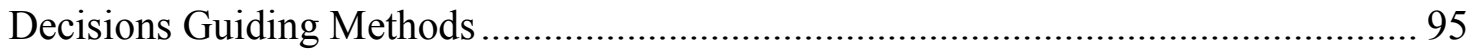

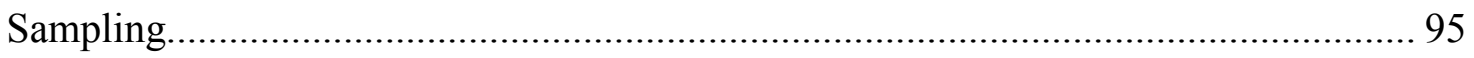

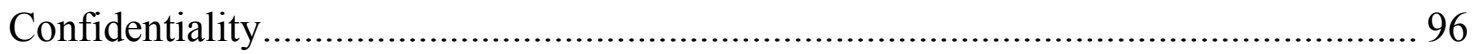

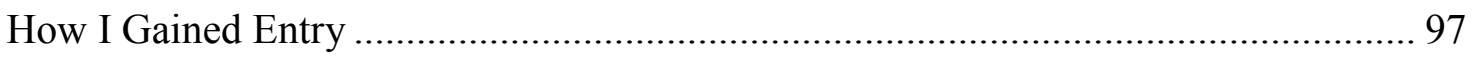

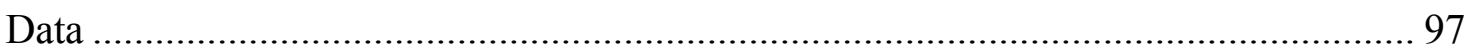

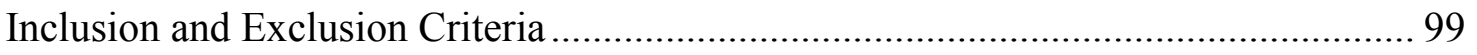

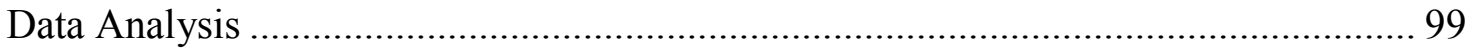

Reliability and Verification in Data Analysis ......................................................... 102

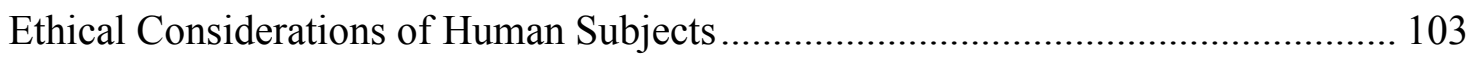

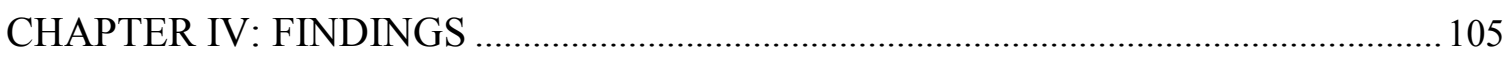

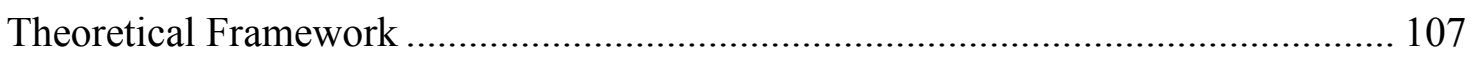

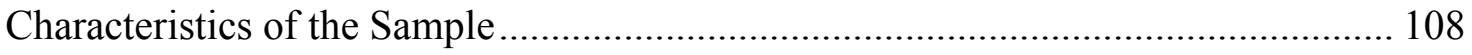

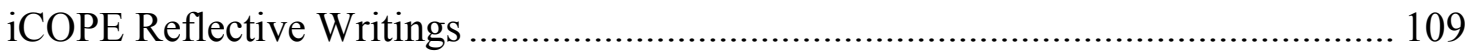

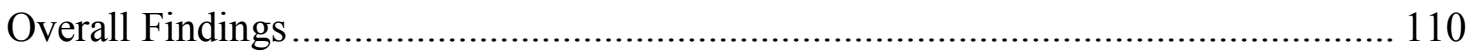

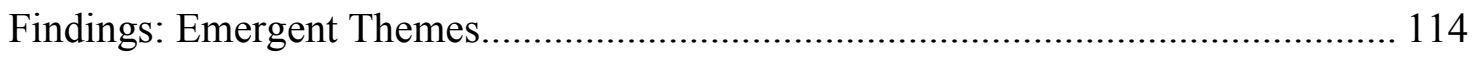

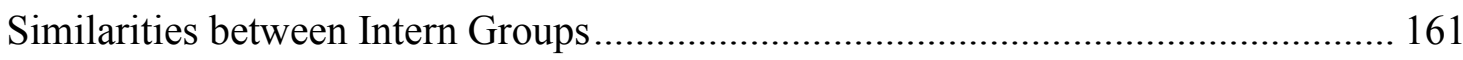

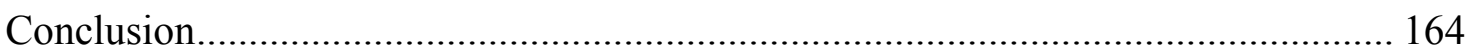

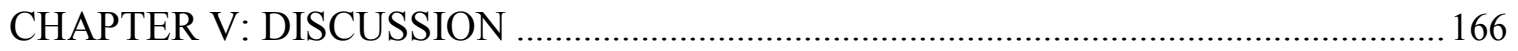

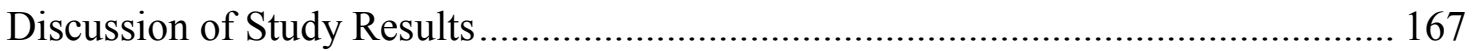

Interdisciplinary Palliative Care Teams ............................................................... 167

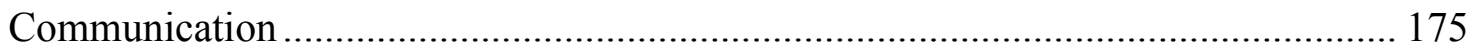

Relevance of Bronstein's Conceptual Model........................................................... 185

Existential Theory and Palliative Care................................................................. 190

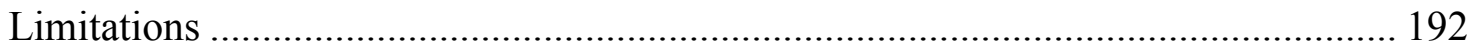

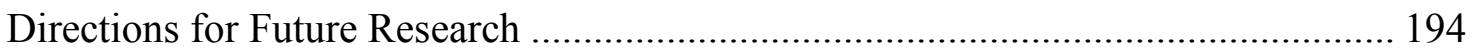

Implications for Future Interdisciplinary Teams and Training ................................... 195

Implications for Counselors and Counselor Education Programs ............................... 196

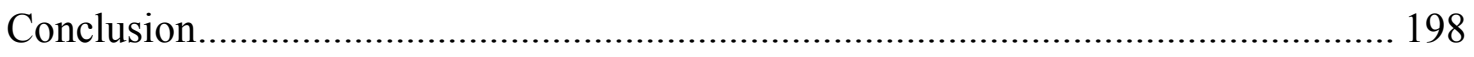

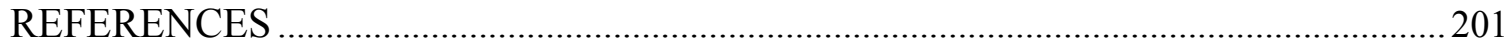

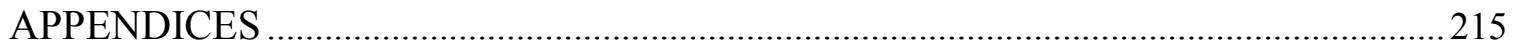

Appendix A: iCOPE Critical Reflection Writing and Group Experience.................... 216

Appendix B: Subject Informed Consent Document...................................................... 218 


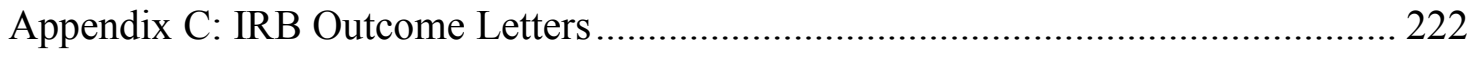

Appendix D: Chaplain Intern Attribute Codes......................................................... 224

Appendix E: Social Work Intern Attribute Codes ................................................... 225

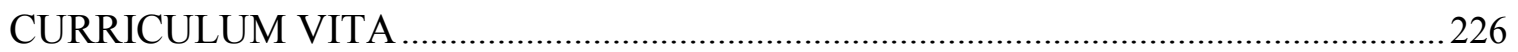




\section{LIST OF TABLES}

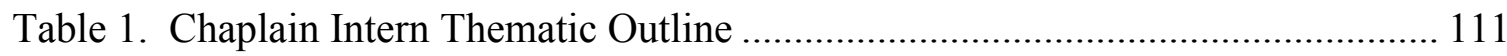

Table 2. Social Work Intern Thematic Outline....................................................... 113

Table 3. Documented Similarities between Chaplain Interns and Social Work Interns 


\section{CHAPTER I: INTRODUCTION}

By 2030, the population of people who are 65 and older is projected to increase significantly up to 72 million representing nearly $20 \%$ of the total U.S. population (Federal Interagency Forum on Aging-Related Statistics, 2012). The projected growth of this elderly population will impact families and healthcare providers as they respond to the needs of this group. Issues that confront this older demographic of Americans are medical care choices, caregiving, changes in living arrangements, financial worries, and decisions about the end-of-life (Maples \& Abney, 2006). Importantly, an increase in endof-life care will be necessary for the growing large group of elderly in the United States.

In 2010, nearly 1.8 million deaths occurred to those ages 65 and over (U.S. Department of Health and Human Services, 2013) and 32\% of deaths in 2009 took place while the deceased was a hospital inpatient (Federal Interagency Forum on AgingRelated Statistics, 2012). The accumulated research suggests the dying are in need of specialized interdisciplinary care; however, major barriers exist. For example, lack of understanding and information, inadequate finances, and anxiety about palliative care impede care for those who are at the end-of-life (Finestone \& Inderwies, 2008).

Deficiencies, such as patient desire for complete honesty and openness from physicians about their diagnosis and prognosis, physicians' reluctance to be truthful with patients, and physician residents' lack of concern about patient' emotional needs, were first examined over 35 years ago (Wilson, Ajemian, \& Mount, 1978).

Significant failings in the care and treatment of hospitalized adults, such as inadequate patient-physician communication and level of patient pain, were found in a 
large two-part study 20 years ago (Connors et al., 1995). More recent studies show improvements in end-of-life care; however, substantial deficits still remain (Bakitas et al., 2009; Gade et al., 2008). For example, deficiencies related to effective communication occur on palliative care interdisciplinary teams (Goldsmith, Wittenberg-Lyles, Rodriguez, \& Sanchez-Reilly, 2010; Mazor et al., 2012), discipline-specific roles interfere with high quality palliative care (Bronstein, 2003; Junger, Pestinger, Elsner, Krumm, \& Radbruch, 2007; Klarare, Hagelin, Furst, \& Fossum, 2013; Nancarrow, Booth, Ariss, Smith, Enderby, \& Roots, 2013; Wittenberg-Lyles, Oliver, Demiris, Baldwin, \& Regehr, 2008), and lack of a common language among diverse disciplines also challenge optimal palliative care delivery (Gachoud, Albert, Kuper, Stroud, \& Reeves, 2012; Mellor, Hyer, \& Howe, 2002).

Twenty years ago, research was conducted on the care provided to seriously ill hospitalized adults. The Study to Understand Prognoses and Preferences for Outcomes and Risks of Treatment (SUPPORT) was a two-year investigation by Connors et al. (1995) designed to improve decision making at the end-of-life and reduce painful and prolonged dying for hospitalized adults. The SUPPORT study (Connors et al., 1995), was conducted in two phases and was composed of a sample of 9,105 hospitalized adult patients with one or more life-threatening diagnoses in five teaching hospitals in the United States.

The Phase I observation of the SUPPORT study found considerable failings (e.g., communication with nurses and physicians, frequency of aggressive treatment, and hospital death difficulties) for seriously ill hospitalized adult patients. Phase II interventions (e.g., nurse-facilitated communication with family for treatments and 
decisions and patient-physician communication, and physician knowledge of patient symptoms and preferences) were chosen in response to the shortcomings found in Phase I. Unfortunately, the Phase II interventions did not improve patient care or outcomes. For example, families reported half of the communicative patients spent their last days of life in moderate or severe pain (Connors et al., 1995). These results describe a deleterious situation for those hospitalized at the end-of-life.

The SUPPORT study results demonstrated the need for a new commitment and more creative efforts to influence the treatment process, as well as the need for substantive changes in the methods used to care for the dying (Connors et al., 1995). Important systems and procedures in end-of-life care have occurred since the SUPPORT study. Hospital-based palliative care programs were developed in order to improve symptom management (i.e., pain reduction), help patients and families understand diverse diseases, clarify patient treatment options, and establish plans for short and long-term care (Gade et al., 2008).

Palliative care emphasizes quality of life and functional status of the patient and family (Goldsmith et al., 2010). Living with advanced incurable disease affects all aspects of the patient's life, causing physical, psychosocial, spiritual, and existential concerns, which requires various discipline-specific professionals to meet the needs of the patient and family (Haugen, Nauck, \& Caraceni, 2010). High quality palliative care involves an organized interdisciplinary team approach, especially interdisciplinary teamwork and communication (Hartrick-Doane, Stajduhar, Causton, Bidgood, \& Cox, 2012). 
Interdisciplinary palliative care teams are typically composed of members from medicine, nursing, social work, and chaplaincy. Research that examines the ways in which psychosocial (e.g., non-medical) interdisciplinary palliative care team members provide care is needed. Role blurring for psychosocial interdisciplinary palliative care team members can be challenging and psychosocial team members struggle to find roles on the interdisciplinary palliative care team by claiming unique expertise (O'Connor \& Fisher, 2011).

Chaplains often experience role conflicts with other interdisciplinary team members, particularly social workers (Wittenberg-Lyles et al., 2008). Interdisciplinary team training is in its early stages and research about interdisciplinary team communication is still needed. In a qualitative study of interdisciplinary palliative care team narratives, Goldsmith, Wittenberg-Lyles, Rodriguez, and Sanchez-Reilly (2010) found team members experienced collaboration and separation as well as significant communication challenges within the interdisciplinary care team.

Coordinated interdisciplinary teamwork is difficult and improving communication with all interdisciplinary team members does not always occur. In an empirical study by Mazor et al. (2012), "breakdowns" in care were found to be fairly common with patients during cancer treatment. Patients were interviewed via telephone and included in the study if they believed something had gone wrong with their care that could have been prevented or could have caused harm. Out of the study sample of 78 patients, $28 \%$ reported a problem in medical care (e.g., delay in treatment), $47 \%$ described a communication problem (e.g., difficulty with information exchange), and $24 \%$ experienced both medical care and communication issues. Mazor et al. (2012) found a) 
patient-perceived communication failures were common, b) patient-perceived communication failures were physically and emotionally damaging to patients, and c) open communication between the patient and the person responsible for the problem with care was the exception.

Zwarenstein, Goldman, and Reeves (2009) recommend interdisciplinary communication and collaboration be studied by qualitative methods in order to have a better understanding of what contributes to effective interprofessional collaboration on teams. When different healthcare professionals work well together, the quality of patient care they provide can be affected; therefore, working well together may improve patient care.

A study by Hartrick-Doane, Stajduhar, Causton, Bidgood, and Cox (2012) examined interdisciplinary team communication. While previous studies observed whether or not teams were communicating, Hartrick-Doane et al. (2012) moved beyond yes or no answers regarding communication. Subtle communication within interdisciplinary teams was analyzed to observe any tensions that could affect optimal communication on interdisciplinary teams. The research from Hartrick-Doane et al. (2012) found communication on the team was negatively affected by the dominance of biomedical knowledge and hierarchy, which caused other team members to feel invisible or be an "unknower" as far as possessing patient information. This interdisciplinary knowledge hierarchy, consisting of "knowers" (i.e., those who possess information) and "unknowers" (i.e., those who do not possess information) and the distribution of knowledge affect communication. 
The study by Mazor et al. (2012) demonstrates a patient-perceived communication failure can cause significant damage. Communication among the interdisciplinary palliative care team members and from the team to the patient and family is critical. Effective communication and positive patient care are very important at the end-of-life.

Consensus exists among health care organizations, research communities, and academics that basic education for clinical professionals should include the knowledge, skills, and attitudes needed for effective interdisciplinary team participation and this education should be maintained within the practice setting (Ponte, Gross, MillimanRichard, \& Lacey, 2010). It is crucial for students preparing to work in today's health care settings to gain interprofessional education (Head et al., 2014). This type of education develops the basis for effective teamwork (Sargeant et al., 2008).

The current study is comprised of beginning professionals who are often part of interdisciplinary palliative care teams. More specifically, the current study will be an examination of chaplain intern and social work intern documented clinical experiences as they participate on an interdisciplinary palliative care team. Typically missing from these teams are representations of non-medical disciplines, such as psychologists, pharmacists, and counselors; therefore, the current study focuses on clinicians from the theological and psychosocial professions. The current study will add to interdisciplinary team practice using the intern written reflections of communication, especially in the area of communication failures. Knowledge and experience with open communication can provide a model for the interns' future practices. 


\section{Conceptual Framework}

The conceptual framework for the current study is the two-part model of optimal interdisciplinary collaboration from Bronstein (2003). Healthcare providers (e.g., physicians and nurses) work together to manage patients, and in the area of palliative care, chaplains and social workers are added to the healthcare team for comprehensive care of the patient. Effective interdisciplinary team characteristics are competence, communication, and organization (Klarare et al., 2013), including leadership, clear vision, quality of care, respect for team roles, and individual characteristics (e.g., listening skills, desire to achieve group goals, and interdisciplinary team experience; Nancarrow et al., 2013).

Bronstein's (2003) two-part model of optimal interdisciplinary collaboration describes generic components for positive collaboration between social workers and other disciplines. The first part of the model lists five core interprofessional processes, which embody team collaboration: interdependence, newly created professional activities, flexibility, collective ownership of goals, and reflection on process. The second part of the model describes influences on interdisciplinary collaboration: professional role, structural characteristics, personal characteristics, and history of collaboration. When these influences exist, interdisciplinary collaboration is supported; when they are missing, barriers occur which impede effective interdisciplinary collaboration.

This two-part model of optimal interdisciplinary collaboration from Bronstein (2003) provides a conceptual structure for effective team communication. Current social work practice often requires collaboration between social workers and others in various settings (e.g., schools, hospitals, and prisons). Bronstein (2003) puts forth this model as a 
plan to create a culture of effective collaboration in numerous settings, which may be used by many disciplines.

\section{Theoretical Framework}

The broad theoretical underpinning for the current study is existentialism.

Existentialism asks deep questions about the nature of human beings and the four givens of existence: death, freedom, isolation, and meaninglessness (Yalom, 1980). Death is the most obvious ultimate concern to the living and to the dying, and is appropriate as a lens for the current study.

Death is defined as a boundary situation (e.g., an urgent experience that forces one to confront an existential state) and one's most powerful boundary situation is confrontation with one's own death (May \& Yalom, 1995). Palliative care provides support as the dying confront their most intensely feared boundary situation. May and Yalom (1995) point out confrontation with a boundary situation (e.g., death) may bring about a shift in perspective of those who were close to the lost loved one, and they may experience growth as a result. The anxiety of death adds an intensity to life and reminds the living that as long as one is alive, one may make changes (Yalom, 1980).

Many individuals who are at the end-of-life are searching for meaning in their remaining time. Frankl (2006) states meaning may be found even when one is faced with a hopeless situation, because when the situation cannot be changed, one is challenged to change oneself. Existentialism is concerned with the here and now, the world of the patient, awareness of time, and the quality of transcendence (i.e., experience beyond the normal level; May \& Yalom, 1995). Death often creates an urgency to live by ignoring the trivial and living authentically (Tomer \& Eliason, 2008). The aspects of existentialism 
are suitable as a theoretical framework to view the needs of the dying and also those who care for them (e.g., the family and the interdisciplinary palliative care team).

\section{Problem Statement}

By 2030, the population of people ages 65 and older is projected to grow significantly_up to 72 million — representing nearly $20 \%$ of the total U. S. population (Federal Interagency Forum on Aging-Related Statistics, 2012). This group comprises the largest number of people ever born in a single generation (i.e., 18 years; Maples \& Abney, 2006). End-of-life care will be needed for many in this large group of adults.

To care for aging adults, healthcare and other service providers will need to be prepared. Many interdisciplinary palliative care teams will be necessary to provide quality end-of-life care. In order to be useful team members for these adults, the United States will require ready and competent workers. Current and future interdisciplinary palliative care team members (e.g., physicians, nurses, chaplains, social workers, and counselors) should be competent in communication skills, interprofessional collaboration, effective teamwork, and symptom management (Institute of Medicine [IOM], 2014). Although counseling programs do not emphasize palliative care education currently (see Council for Accreditation of Counseling and Related Education Programs [CACREP], 2009), information about interdisciplinary palliative care practices will have relevance for this discipline as well.

Communication is considered to be the essential factor for interdisciplinary team efficacy (Sargeant, Loney, \& Murphy, 2008). Collaboration is the central dynamic of interdisciplinary team work (Bronstein, 2003). If there are problems in the ways team members communicate and work together, problems may occur in patient care 
(Zwarenstein, Goldman, \& Reeves, 2009). Clinicians are expected to have frequent conversations with their patients about end-of-life values and goals; however, many people, typically those who are poorer, younger, minority, and less-educated, do not talk about their end-of-life preferences (IOM, 2014).

High quality palliative care requires a coordinated interdisciplinary team approach; however, substandard communication and team collaboration are constant challenges (Hartrick-Doane et al., 2012). Effective interdisciplinary team functioning does not happen without effort and awareness (O’Connor \& Fisher, 2011). Interdisciplinary teams require work to become effective and this necessary work is not typically recognized by the healthcare profession (Sargent et al., 2008).

As described later in Chapter 3, all participants in this study are chaplain interns and social work interns. Their documented experiences in response to a series of established questions for the Interdisciplinary Curriculum for Oncology and Palliative Education [iCOPE] study (see Appendix A) will serve as the primary data source for this dissertation study. Thus, pre-existing data (e.g., documents and text) from chaplain interns and social work interns about their interdisciplinary palliative care team experience will be used in the current study to explore the research questions.

\section{Research Questions}

The research questions to be addressed are as follows.

\section{Research Question 1}

What is the nature of working on an inpatient hospital unit interdisciplinary palliative care team for chaplain interns and social work interns?

The sub-questions associated with this research question are: 
a) What are the documented interdisciplinary team communications and experiences for chaplain interns?

b) What are the documented interdisciplinary team communications and experiences for social work interns?

c) How do the chaplain interns document their communications and experiences with patients and families who are receiving inpatient hospital unit interdisciplinary palliative care?

d) How do the social work interns document their communications and experiences with patients and families who are receiving inpatient hospital unit interdisciplinary palliative care?

\section{Research Question 2}

In what ways does the inpatient hospital unit interdisciplinary palliative care team influence the chaplain interns and social work interns as they work with dying patients and their families?

The sub-questions associated with this research question are:

a) What are the documented thoughts, emotions, and feelings of the chaplain interns as they reflect upon the meaning of their interdisciplinary palliative care experience?

b) What are the documented thoughts, emotions, and feelings of the social work interns as they reflect upon the meaning of their interdisciplinary palliative care experience?

c) How are the described roles and behaviors of the chaplain interns shaped by their interdisciplinary palliative care experience? 
d) How are the described roles and behaviors of the social work interns shaped by their interdisciplinary palliative care experience?

\section{Research Question 3}

How similar are the documented experiences, behaviors, and feelings of chaplain interns to those documented experiences, behaviors, and feelings of social work interns working in inpatient hospital unit interdisciplinary palliative care teams?

\section{Need for the Study}

A dearth of empirical research exists to support the interdisciplinary model of care. Systematic reviews from Zimmerman, Riechelmann, Krzyzanowska, Rodin, and Tannock (2008) and Zwarenstein et al. (2009) found methodological limitations, small number of empirical studies, sample sizes, multiple outcome measures, and variety of settings and interventions made the generalization of interdisciplinary team effectiveness difficult. Other systematic reviews determined interdisciplinary teams improved family satisfaction (Hearn \& Higginson, 1998; Zimmerman, Riechelmann, Krzyzanowska, Rodin, \& Tannock, 2008). In one study there was an advantage of multidisciplinary teams over unidisciplinary care teams (Finlay et al., 2001). A meta-analysis demonstrated evidence of a range of benefits to patients who were treated by an interdisciplinary palliative care team (Higginson et al., 2002).

As interdisciplinary teamwork becomes the standard of care (Remke \& Schermer, 2012) in many areas of healthcare, including the model of quality palliative care provision (IOM, 2014; World Health Organization [WHO], 2003), more empirical studies may be helpful for educational curriculum decisions. A study of reflective writings from a group of chaplain interns and social work interns may provide insight 
into their learning experiences. The interns' understandings may also help their faculty with curricular decisions within interprofessional education courses.

Team communication is essential to effective interdisciplinary team functioning (Bokhour, 2006; Goldsmith et al., 2010; Junger et al., 2007; Sargeant et al., 2008). Interns who have clinical placements on interdisciplinary palliative care teams may experience how communication on those teams is conducted. Most important is what the interns learn about team communication on an inpatient hospital unit interdisciplinary palliative care team. Zwarenstein et al. (2009) recommend interdisciplinary communication and collaboration be studied by qualitative methods in order to have a better understanding of how healthcare professionals work well together, which can affect the quality of patient care they provide. The present qualitative study of interdisciplinary palliative care team chaplain and social work interns' clinical placement reflections may offer insights into the ways healthcare professionals communicate.

\section{Significance of the Study}

This study may add to the growing field of interdisciplinary palliative care team clinical placement research and knowledge. More empirical research from experiential clinical interdisciplinary palliative care team placements will add to the knowledge base within academic healthcare programs. As more academic hospitals incorporate interdisciplinary palliative care team programs, the research from the current study may provide insights for the interdisciplinary faculty who oversee programs. Although findings from qualitative research are not generalizable, they may provide helpful information for others who create interdisciplinary palliative care team educational programs. 
Another way in which the current study will add to the research base is to explore existing conceptual models of interdisciplinary communication. The current study will use Bronstein's (2003) conceptual framework of two-part optimal interdisciplinary collaboration. Bronstein's (2003) model will consider whether interdisciplinary collaboration is reported by a select interdisciplinary palliative care team of chaplain interns and social work interns. The current study may be the first to apply the Bronstein (2003) model of interdisciplinary communication to chaplain interns' and social work interns' reflections of clinical placement experiences.

The current study may inform the faculty of the progress of interns as they experience interprofessional team development after they complete their placements on interdisciplinary palliative care teams. Within the IOM (2014) suggestions, under Recommendation 3: Professional Education and Development, endorses educational institutions, among others, to establish training requirements which increase palliative care knowledge and skills of all who care for those at the end-of-life. Recommendations that are especially pertinent to the current study are: all clinicians across the diverse disciplines who work with those at the end-of-life should be competent in basic palliative care, and this includes communication skills, interprofessional collaboration, and symptom management (IOM, 2014). Currently, palliative care education is lacking in the field of counseling (CACREP Standards, 2009).

Of particular note, academic medical centers, teaching hospitals, and other palliative service providers (e.g., hospices) should consider committing institutional resources to increase the number of training positions for specialty-level palliative care (e.g., interdisciplinary palliative care teams) in order to have a ready workforce for the 
projected large group of aging adults. The current study will provide results from chaplain interns and social work interns as they learn how to deliver palliative care from their unique clinical experiences on an interdisciplinary palliative care team. It may provide data which suggest the IOM's (2014) educational policy recommendations for competency in basic palliative care and palliative care placements are in-progress. The interns' interdisciplinary palliative clinical experience may help them learn about communication, interprofessional collaboration, and symptom management. The present study could have applicability for other human helping professions (e.g., counseling).

Policy may be informed from the current study. In a 2014 report from the IOM, Dying in America Improving Quality and Honoring Individual Preferences near the Endof-Life, key findings and recommendations are made (IOM, 2014). These suggestions could enhance quality of life through the end-of-life, as well as support a more helpful palliative system of care.

In particular, the section under Key Findings titled "Professional Education" has relevance to the current study. The IOM (2014) determines the education of health professionals in palliative medicine has improved; however, three problems still remain. These are a) palliative care gets little attention in medical and nursing school curricula, b) education is taught in silos, which prevents interprofessional team development, and c) physicians' lack of communication skills.

This study deepens the literature because national and international agencies have asked that future workforce be ready for interdisciplinary collaboration (IOM, 2014; WHO, 2010). Educational experiences can enhance interdisciplinary team learning and those who have positive clinical placement experiences are better prepared to enter the 
workforce as effective interdisciplinary team members (Bronstein, 2003). Finally, Zwarenstein et al. (2009) recommend qualitative methods be used to determine how interprofessional collaboration changes may change patient outcomes.

\section{Delimitations}

The location of this study will be Louisville, Kentucky and the sample of the study will be the written reflections of clinical interdisciplinary palliative care team experiences from chaplain interns and social work interns. The timeframe of the study will consist of the written reflections from spring and fall semesters from 2012 through spring semester of 2015. Qualitative research findings will not be generalizable to other populations. However, the research that will be collected from the interns' reflections (i.e., documents and text data) will be pertinent to the larger base of knowledge about the communication, interaction, and barriers within an interdisciplinary palliative care team.

\section{Limitations}

Influences that are not controlled in this study are the 10 prompts from the writing reflection (see Appendix A) and the quality of writing. The sample of chaplain interns and social work interns will be geographically limited and may not produce information that could be helpful in other areas of the country. Louisville, Kentucky is a mid-size city and the hospitals represented in the study will be a mixture of academic, private, public, and restricted to veterans.

\section{Definitions of Current Study}

Chaplain interns: Chaplaincy students who are completing a Clinical Pastoral Education residency. 
Hospice: A program that provides comprehensive, interdisciplinary team-based palliative care for dying patients who have a limited time to live and is a program of the United States Federal Government (Medicare and Medicaid Programs: Hospice Conditions of Participation, 2008; Meier, 2011).

Interdisciplinary: Indicates a reciprocal interaction between disciplines, of analysis and synthesis, which requires blurring of disciplinary boundaries so that new knowledge is revealed (Choi \& Pak, 2006).

Interdisciplinary collaboration: The concept that safety, quality, and efficiency in patient care is improved by the processes within the team that views all its members as equals, encourages interdisciplinary teamwork, and reduces the traditional hierarchical systems (Ponte et al., 2010; Sargeant et al., 2008).

\section{Interdisciplinary Curriculum for Oncology and Palliative Education}

[iCOPE] study: This is a program within the University of Louisville, School of Medicine, which originated from an externally funded grant used for palliative care education from the Robert Wood Johnson Foundation. Qualitative data derived from the iCOPE study will be used in the current dissertation study.

Interdisciplinary Palliative Care Teams: For the current study, this is a team composed of physician, nurse, chaplain, and social worker who collaborate to provide patient-centered care and is considered to be the foundation of palliative care. (Hurley, Barg, Strumpf, \& Ersek, 2014; Xyrichis \& Lowton, 2008).

Interprofessional education: Education which involves educators and learners from two or more health professions. These disciplines create and foster a collaborative learning environment. These efforts are to develop knowledge, skills, and attitudes which 
result in interprofessional team behaviors and competence. The goal of interprofessional education is for students to learn how to work on an interprofessional team and take the knowledge, skill, and value into their future practice, ultimately offering interprofessional patient care as part of a collaborative team and focused on patient outcomes (Buring et al., 2009; Head et al., 2014).

Interprofessional teamwork: The levels of cooperation, coordination, and collaboration characterizing the relationships between professions in delivering patient-centered care (Interprofessional Education Collaborative Expert Panel, 2011).

Multidisciplinary: Different disciplines separately working together without challenging discipline-specific training and boundaries (Choi \& Pak, 2006).

Palliative care: Holistic multiprofessional care with skilled assessment and management of symptoms and problems that seeks to alleviate these difficulties and to enable patients to live well for as long as possible, to die with comfort and dignity, and to provide support to the family (Higginson \& Evans, 2010).

Social Work interns: Social work students who are completing a Master's degree.

\section{Outline of the Dissertation}

This dissertation is organized into five chapters, with references and appendixes in the following manner. Chapter 2 introduces a review of related literature that examines palliative care and the evolution of interdisciplinary teams, specifically how they operate when providing palliative care. Chapter 3 explains the research design and methodology of the study. The sample data and procedures to be followed for analysis will be described. Chapter 4 presents data analysis and results. Chapter 5 contains the discussion 
of the findings, summary, conclusions, recommendations of the study, and implications for diverse providers, including the counseling profession. A list of references and appendices closes the study. 


\section{CHAPTER II: LITERATURE REVIEW}

This chapter reviews the empirical and theoretical literature that informs and supports the current study. In this chapter, hospice care, its historical beginning, and current status will be examined. The field of palliative care will also be discussed. Hospice care and palliative care are very similar concepts, and are used as interchangeable terms in other parts of the world, but not in the United States.

Teams, in general, and specifically, interdisciplinary teams, will be reviewed. Foundational terms that generally pertain to teams and their specific descriptions, such as multidisciplinary and interdisciplinary, will be defined. Team composition in healthcare and roles of interdisciplinary palliative care team members will also be clarified. Support from national and international agencies and the empirical studies that sanction the use of interdisciplinary palliative care teams as the standard of care will be discussed.

All roles on the interdisciplinary palliative care team will be reviewed; however special emphasis will be placed on the spiritual and psychosocial interdisciplinary palliative care team professionals because the current study will focus on their communication and interactions with the team. Effective interdisciplinary palliative care teams' characteristics will be described as well as barriers and challenges to their effectiveness will be stated. Communication is the essential ingredient for an interdisciplinary palliative care team; patients and families lose trust in their clinicians when mistakes in their care occur. Finally, conceptual and theoretical underpinnings for interdisciplinary teams will also be considered. 


\section{Hospice}

Hospice is a term that refers to a philosophy of care and also refers to a specific place (i.e., a designated building) for care at the end-of-life (Hurley et al., 2014). Hospice care is a voluntary, time-limited program. Patients generally must have six months or less to live, given the normal course of their disease or health situation, as determined by a physician. Hospice patients are required to end all curative treatment (Meier \& Brawley, 2011) and allow natural death to occur.

Both hospice care and palliative care provide treatment for the dying, however, differences exist between both types of care. Hospice is an agency that provides palliative care, but palliative care is not hospice because it does not operate under the same set of regulations (Rome, Luminais, Bourgeois, \& Blais, 2011). Hospice is designed to provide comprehensive, interdisciplinary team-based palliative care for dying patients who have a limited time to live (Meier, 2011).

The hospice care program of the United States federal government was created in 1983 by The Medicare Hospice Benefit in order to provide Medicare beneficiaries access to high quality end-of-life care (National Hospice and Palliative Care Organization [NHPCO], 2012). Private insurance also pays for hospice care. Once a patient is admitted to a hospice program, the agency provides comprehensive interdisciplinary palliative care for the patient until death.

Although hospice care cannot begin unless a physician determines the patient's life expectancy to be six months or less, patients do not have a limited amount of time they may spend in hospice care (e.g., patients must be re-evaluated at regular intervals to verify continued decline to remain in a program). Hospice care is often provided in the 
patient's home, but it is also delivered in hospice-designated beds within an in-patient hospital unit or skilled nursing facility.

Federal law mandates the services hospice agencies provide and the manner in which they are to be administered, including some flexibility in meeting quality standards (Medicare and Medicaid Programs: Hospice Conditions of Participation, 2008). Medicare hospice agencies must provide each patient with an interdisciplinary team, which typically consists of a physician, nurse, social worker, chaplain, and volunteer (Medicare and Medicaid Programs: Hospice Conditions of Participation, 2008). Currently, there are few programs that include counselors (Babcock \& Robinson, 2011). When patients are admitted to a hospice program, they receive a number of core services, which include nursing care, support with activities of daily living, rehabilitation therapies, psychosocial care, spiritual care, and all medications and durable medical equipment necessary for their end-of-life process (Finestone \& Inderwies, 2008).

Hospice care is unique in its required use of volunteers, which must be $5 \%$ of total patient care hours (NHPCO, 2012). Another core requirement of any hospice program is bereavement support for the family. Hospice bereavement care may be offered as individual therapy, psycho-educational groups, or support groups. Grief assistance from therapists within the hospice bereavement department is available and free of charge to family members for a minimum of 12 months after the loved one dies (NHPCO, 2012).

The National Hospice and Palliative Care Organization (NHPCO) is the hospice membership organization that participated in the creation and implementation of the National Consensus Project for Quality Palliative Care guidelines at the organizational level in 2004 (National Consensus Project for Quality Palliative Care [NCP], 2013). The 
NHPCO supports the NCP guidelines to provide comprehensive care to their patients and families (NHPCO, 2012). Often patients receive hospice care when they are discharged from the hospital to home or a nursing home at the end-of-life. With unifying guidelines in place for any transition in level of care, patients and their families are assured of consistent end-of-life support, whether they spend their final days at home, in the hospital, or in a skilled nursing facility.

\section{History of Modern Hospice Movement}

The modern hospice movement is attributed to Cicely Saunders, who described the evolution of St. Christopher's hospice in London in 1967 (Saunders, 2000) and when hospice services began in the United States in Connecticut in 1974 (Saunders, 1998). St. Christopher's hospice was the first modern hospice, which began as a separate institution established with a small bequest from a young adult patient who died of cancer nearly 20 years earlier. As this patient lay dying in a busy surgical ward, he often spoke with Saunders about the existence of a place where pain could be relieved and one could contemplate the end-of-life (Saunders, 2000).

Nearly concurrent with the beginning hospice services in the United States, Balfour Mount, a Canadian physician, began plans for palliative care provision. He was dismayed to see dying patients isolated, viewed with suspicion and distrust, as a result of a lack of communication between patients, family, nurses, and physicians (Mount, 1976). The appropriate goal for the dying is treatment that will promote the giving of optimal quality of life for both patient and family. Mount (1976) was also a proponent of a team approach for improved patient quality of life. He found the option of a hospital palliative care unit, staffed by interdisciplinary team, to produce a positive and creative attitude 
toward death and bereavement (Mount, 1976) and the key to successfully meeting the needs of the terminally ill (Wilson et al., 1978).

In her work with the dying, Saunders (2000) created and refined hospice basic principles, which remain as core requirements today (Medicare and Medicaid Programs: Hospice Conditions of Participation, 2008). These principles are a) symptom control for the patient, b) patient care provided by multiprofessional team, c) the patient and family are the unit of care, d) spiritual needs may be paramount, and e) bereavement services are provided for survivors, including children (Saunders, 2000). The recognition that a dying patient's quality of life requires care for physical, psychosocial, and spiritual needs has infiltrated much of today's medicine (Doka, 2009).

\section{Hospice Care Today}

Although the core principles of hospice have not changed, federal funding and reimbursement of services are essential to this program. Hospice care was designed to allow the patient to remain at home as long as possible, help the patient remain comfortable, and maintain dignity, including quality of life (Medicare and Medicaid Programs: Hospice Conditions of Participation, 2008). Most hospice patients receive care in their homes; however, they are increasingly dying in hospice inpatient facilities because families are unable to manage patient symptoms at home (Hurley et al., 2014).

In a statement on data from the National Hospice Study, Mor and Birnbaum (1983) discussed the implications of Medicare legislation as it concerns reimbursed hospice services. Core services for patients stipulated by this legislation are nursing, medical social services, physician direction of the hospice team, counseling, and volunteer services (Mor \& Birnbaum, 1983). In an early examination of hospice services, 
the National Hospice Study, Mor and Kidder (1985) found no case where hospice care was harmful or more costly and concluded hospice care to be an economical option for terminally ill cancer patients.

Another empirical study by Taylor, Ostermann, Van Houtven, Tulsky, and Steinhauser (2007) also determined hospice use reduced Medicare expenditures during the last year of life. In addition, Meier (2011) reported access to hospice and palliative care programs is variable across the United States. Going forward, more hospice and palliative care services will be necessary to treat the large group of those over 65 years of age, whose population is expected to increase dramatically by 2030 (Federal Interagency Forum on Aging-Related Statistics, 2012).

\section{Palliative Care}

Palliative care emphasizes relief of pain and suffering of a patient with lifethreatening illness. The World Health Organization (WHO) defines palliative care as an approach that improves the quality of life of patients and their families facing the problem associated with life-threatening illness, through the prevention and relief of suffering by means of early identification and impeccable assessment and treatment of pain and other problems, physical, psychosocial, and spiritual (WHO, 2003). Another definition for palliative care is holistic multiprofessional care with skilled assessment and management of symptoms and problems that seeks to alleviate these problems and to enable patients to live well for as long as possible, to die with comfort and dignity, and to support the family (Goldsmith et al., 2010; Higginson \& Evans, 2010; Zimmerman et al., 2008). Doyle, Hanks, and MacDonald (1998) added "palliative care is planned care, not crisis intervention" (p. 6). 
The palliative care provider's role is to relieve the suffering of patients and their families with comprehensive assessment and treatment of physical, psychosocial, and spiritual symptoms (Rome et al., 2011). Today's aging patients have chronic illnesses which may last for years, may be accompanied by a reduced quality of life, and often require more medical interventions and care (Bookbinder \& McHugh, 2010). The rapid growth of hospital palliative care teams has been in response to provide care for the group of patients who do not meet the hospice eligibility requirement of dying within six months (e.g., the seriously ill who are not dying soon; Meier, 2011).

Long-term conditions require more than a single discipline to provide care and the multiple discipline approach to chronic illness has been suggested to be the most comprehensive way to provide care (Xyrichis \& Lowton, 2008). The manner in which the patient in palliative care is treated is often by an interdisciplinary team, which is the foundation and central component of palliative care (Junger et al., 2007; Meier \& Beresford, 2008). The interdisciplinary team is responsible for patient and family comprehensive care from intake until death and bereavement care (O'Connor, Fisher, \& Guilfoyle, 2006).

\section{Palliative Care: Conditions for Care}

The National Consensus Project for Palliative Care, whose purpose is to promote clinical practice guidelines to ensure consistent and high-quality care, and guide development of new and existing palliative care services, describes the conditions that are appropriate for palliative care (NCP, 2013). Palliative care is suitable management of chronic, serious, and life-limiting illnesses, such as cancer, heart disease, chronic obstructive pulmonary disease (COPD), kidney failure, dementia, acquired immune 
deficiency syndrome (AIDS), amyotrophic lateral sclerosis (ALS), multiple sclerosis (MS), and acute stroke (NCP, 2013). Palliative care may also be prescribed to those in life-threatening situations as a result of a catastrophic event, such as an accident or other trauma, congenital abnormality, or condition that will result in dependence on lifesustaining treatment or death (NCP, 2013).

Patients who have multiple illnesses or other complex conditions may receive palliative care. The American Society of Clinical Oncology (Smith et al., 2012) suggested consideration of integrating palliative care with standard oncology care at the time of the initial diagnosis. This provisional clinical opinion is based upon empirical evidence from several clinical trials, including Bakitas et al. (2009) and Gade et al. (2008).

The randomized clinical trial from Bakitas et al. (2009) found quality of life and mood were improved with a palliative-care focused intervention that concentrated on physical, psychosocial, and care coordination. The American Society of Clinical Oncology also based its suggestion to begin palliative care on a study of interdisciplinary palliative care service patients from Gade et al. (2008). Gade et al. (2008) found patients who received interdisciplinary palliative care reported greater satisfaction with their care experiences and with providers' communication.

\section{Palliative Care: Recipients}

Palliative care is appropriate for patients of all ages and conditions. Those who may receive palliative care are neonates, children, adolescents, as well as adults. It is also suitable for children, adolescents, and adults with developmental and intellectual 
disabilities, including vulnerable and underserved populations, such as the homeless, immigrants, veterans, prisoners, and those with serious mental illness (NCP, 2013).

As the impact of a child's death is unmistakable, children often receive both curative and palliative care (Connor, 2009). The American Academy of Pediatrics' policy statement considers pediatric palliative care to be essential care of infants, children, adolescents, and young adults with life-shortening conditions, including their families. These treatments are to relieve suffering across the physical, psychological, social, practical, and spiritual realms and should be provided as collaborative interdisciplinary multimodal care (American Academy of Pediatrics, 2013).

In an empirical study of pediatric palliative care, Hays et al. (2006) found several care processes that blended curative and comfort care to improve parent perceptions and parent satisfaction of their child's quality of life. A key factor is effective communication between the parents and providers that support patient preferences. Communication within the pediatric palliative care context requires contact among many sources (e.g., insurance providers, medical professionals, psychosocial clinicians, and spiritual experts), which can be challenging. However, results from the study suggest patient quality of life and family satisfaction for children with life-threatening illness can be improved with integrated pediatric palliative care (Hays et al., 2006).

\section{Palliative Care: Timeframe}

Palliative care may be either long-term or short-term, depending on the needs of the patient; there is no time limit for providing palliative care. Palliative care may be utilized from the time of diagnosis until the end-of-life. The American Society of Clinical Oncology Provisional Clinical Opinion (Smith et al., 2012), Institute of Medicine report 
(2014), and American Academy of Pediatrics (2013) policy statements recommended palliative care be offered with standard oncology care to patients. However, it must be recognized these recommendations are non-binding.

\section{Palliative Care: Locations}

Palliative care delivery occurs in the patient's specific care setting, such a hospital, skilled nursing facility, assisted living facility, or at home (Connor, 2009). As the patient's condition changes, or worsens, a higher level of care may be required (e.g., the patient's home to a skilled nursing facility). Palliative care is continuous care and is transferable from one level of care to another (e.g., the patient's home to an in-patient hospital unit).

Hospice care was designed to be provided in the home; however, care is often delivered in both home and inpatient facilities. A study by Hurley, Barg, Strumpf, and Ersek (2014) interviewed 24 interdisciplinary team members and found unique differences between inpatient hospice care and home hospice care. Home safety concerns were most important for the home hospice interdisciplinary team members (e.g., ease of exiting in case of an emergency and the risk of falling); other concerns involved medication administration and the availability of a competent caregiver to provide patient care. Inpatient hospice interdisciplinary team members focus on crisis management and assume responsibility for the patient's direct care.

Hurley et al. (2014) found a lack of continuity within the plan of care between locations of care (e.g., home hospice or inpatient hospice unit). Each location of care presents distinct challenges to the interdisciplinary palliative care team and the family; the family balances the desire to remain at home with the need for inpatient symptom 
management (Hurley et al., 2014). The study highlights a small part of the complex nature of end-of-life care in the United States.

\section{Type of Teams}

A team may be defined as a specific structural unit of a small number of reliable members who are mutually accountable for team outcomes (McCallin, 2001). The WHO defines a team as "two or more people working interdependently towards a common goal" (WHO, n.d., p. 4). Sargeant et al. (2008) describe a team as a group who achieves tasks through interdependence and collaborative work of its members. Team members work interdependently, which means they know the team and what each can contribute to their common goal (Haugen et al., 2010).

\section{Teams: Composition}

Teamwork is a fundamental element of palliative care (Haugen et al., 2010). Health care professionals have been using teamwork as the preferred method for planning and monitoring patient goals since the mid-1970's (Fiorelli, 1988). Teamwork is the most effective way to achieve complicated tasks and can enhance patient care delivery (Youngwerth \& Twaddle, 2011). Evidence suggests teams of different professional disciplines, working effectively and cooperatively together, can improve patient care outcomes and achieve goals (McCallin, 2001; Zwarenstein et al., 2009).

\section{Teams: Structure, Communication, and Collaboration}

The literature on interdisciplinary teams discusses collaboration exclusive of defining the make-up of the team. The application of the terms multidisciplinary and interdisciplinary are used to describe team composition of different disciplines rather than how the team functions (Youngwerth \& Twaddle, 2011). Nancarrow, Booth, Ariss, 
Smith, Enderby, and Roots (2013) described how the terms interdisciplinary, interprofessional, multiprofessional, and multidisciplinary are used interchangeably to refer to team processes. "Interdisciplinary team" is a generic term of reference for healthcare teams that are composed of professionals from different disciplines (Nancarrow et al., 2013).

In a literature review, Choi and Pak (2006) defined the terms multidisciplinary and interdisciplinary, which are frequently used to describe healthcare teams. The authors proposed these terms refer to involvement of multiple disciplines, which vary along a continuum. Multidisciplinary uses knowledge from different disciplines without challenging disciplinary boundaries; whereas interdisciplinary indicates a reciprocal interaction between disciplines of analysis and synthesis, and requires blurring of disciplinary boundaries so that new knowledge is revealed (Choi \& Pak, 2006).

Effective team members are able to express themselves within the team and with the patient and family. Communication and collaboration among team members are vital for providing quality palliative care. The Institute of Medicine recommended "all clinicians, across disciplines and specialties who care for those with advanced serious illness should be competent in basic palliative care management, including communication skills, interprofessional collaboration, and symptom management" (IOM, 2014, p. 4). Junger et al. (2007) determined close communication to be a crucial aspect for effective team work. In their qualitative study of team work with interprofessional focus groups, Sargent, Loney, and Murphy (2008) found communication to be the essential factor in an effective team. 
Effective communication comes before collaboration; factors that promote collaboration include respect, sharing, and trust (Henneman, Lee, \& Cohen, 1995). Language used by the team is also an indication of team communication. According to Henneman et al. (1995), assessment of the frequency of use of "we" versus "I" statements between health care professionals is an easy way to note the workings of a team-not an individual—process.

Effective collaboration is a synergy that relies on team members' confidence in team interactions to be successful with essential responsibilities (Parker-Oliver, Bronstein, \& Kurzejeski, 2005). Bronstein (2003) noted collaboration to be the central dynamic of interdisciplinary work and is supported by professional roles, team structure, personal characteristics of members, and the team's history of collaboration. In their study of interdisciplinary palliative care team members' interviews, Klarare, Hagelin, Furst, and Fossum (2013) found team collaboration requires conscious effort and is vital within the team and with the patient and family. Cooperation between team members is important for collaboration and communication within the team, which translates into useful communication for the patient and their treatment goals. Collaborative communication recognizes "the essential reciprocity and dynamic synergy of this pair of concepts, whereby better communication enhances collaboration, and more skillful collaboration can improve communication" (Feudtner, 2007, p. 584).

\section{Interdisciplinary Palliative Care Teams}

Interdisciplinary teams are the foundation of palliative care (Hurley et al., 2014; Xyrichis \& Lowton, 2008). The needs of the dying are so broad and diverse that an interdisciplinary team comprised of multiple disciplines is used to manage the care of the 
palliative patient (McCallin, 2001; Xyrichis \& Lowton, 2008). Different professional disciplines collaborate to provide a comprehensive and "whole person" approach to the physical, psychosocial, and spiritual requests of patients who are coping with lifethreatening illness (Meier \& Beresford, 2008).

The model of interdisciplinary care is reflected during a team's collaborative discussions when the team members participate in the patient's treatment decisions (Bokhour, 2006). Hartrick-Doane et al. (2012) stated the "palliative approach is dependent on coordinated care between interprofessional team members" (p. 2). Palliative care programs use an interdisciplinary approach to provide care that focuses on the patient's quality of life and ability to function within daily life, as well as on the patient's family (Goldsmith et al., 2010). As their condition worsens, patients must cope with progressively difficult physical symptoms and challenging psychological and social issues that require constant medical and nursing attention; ideally this complex care is provided by interdisciplinary palliative care staff (Peters \& Sellick, 2006).

From their study of interdisciplinary team functioning, Parker-Oliver, Bronstein, and Kurzejeski (2005) found the team comprised of physician, nurse, social worker, and chaplain to be the ideal model for treatment. Rome, Luninais, Bourgois, and Blais (2011) suggested optimal pain relief occurs when the patient's total pain (i.e., the sum of pain in the physical, psychosocial, and spiritual domains) is addressed, which includes using social workers and chaplains to reduce suffering. Interdisciplinary teamwork uses a comprehensive approach recommended to provide patient-centered care as healthcare teams combine their experience, skills, and knowledge to produce enhanced results (Youngwerth \& Twaddle, 2011). 
Several national palliative care organizations make recommendations for the delivery of palliative care. The NCP was formed in 2004 to create guidelines for comprehensive and high-quality palliative care service along with the National Quality Forum, which is a private, nonprofit membership organization that developed a national strategy for quality healthcare improvement (NCP, 2013). The NCP and the National Quality Forum Preferred Practices consider palliative care that is provided by an interdisciplinary team of palliative care professionals one way to achieve high-quality healthcare (Youngwerth \& Twaddle, 2011). The interdisciplinary palliative care team is to deliver patient and family centered interdisciplinary collaborative palliative care, possess clinical and communication proficiency among the team members, and provide relief of physical, psychological, emotional, and spiritual suffering, which includes distress of patients and their families (NCP, 2013).

\section{Interdisciplinary Palliative Care Teams: Standard of Palliative Care}

The interdisciplinary model of care has become the gold standard of palliative care treatment (Remke \& Schermer, 2012). National and international organizations consider the interdisciplinary team as providing high quality palliative care (IOM, 2014; National Association of Social Workers Standards for Palliative \& End-of-life Care, 2004; NCP, 2013; WHO, 2003). Empirical evidence supports interdisciplinary teams to improve patient outcomes (Gade et al., 2008); however, other types of palliative care, (e.g., medical and nurse clinicians only) have also demonstrated benefit to patients receiving palliative care (Bakitas et al., 2009; Dionne-Odom et al., 2014).

Systematic reviews have reported on methodological difficulties such as small number of studies, sample sizes, variety of outcome measures, and heterogeneity of 
interventions and settings, which make it difficult to ascertain key elements of interdisciplinary team effectiveness (Zimmerman et al., 2008; Zwarenstein et al., 2009). Other systematic reviews have determined interdisciplinary palliative care teams benefit patients and families. Hearn and Higginson (1998) found evidence that interdisciplinary teams improve satisfaction of patient and family needs as compared to conventional care.

Palliative care is delivered in many locations and studies measure many different outcomes. Limited evidence was found to support hospital-based palliative care teams from Higginson et al. (2002). A review by Finlay et al. (2002) supported the effectiveness of palliative care teams, which include a benefit of multidisciplinary teams over unidisciplinary teams. Evidence of improved family satisfaction with care was shown from palliative care teams (Zimmerman et al., 2008). Lastly, a meta-analysis from Higginson et al. (2002) showed the strongest evidence of benefit to patients to be palliative care team intervention for pain and symptom control.

More systematic reviews have demonstrated increased evidence in favor of using interdisciplinary palliative care teams to provide comprehensive palliative care. Higginson and Evans (2010) demonstrated inpatient palliative care teams significantly improve patient outcomes of pain, symptom control, and anxiety. In their review, Leclerc et al. (2014) provided preliminary evidence that interdisciplinary palliative care teams can improve patient satisfaction and the funding and development of palliative care teams is one way to increase patient care.

Research has shown how interdisciplinary palliative care teams improve patient outcomes; however, there are empirical studies which have supplied information about the lack of quality of end-of-life care. In one study, Teno et al. (2004), through telephone 
surveys, learned about the concerns of family members or knowledgeable others of 1578 decedents, of which $67.1 \%$ (i.e., 1059) were in institutional care (e.g., hospital or nursing home) or at home (i.e., 519) at the time of death. About $25 \%$ of the survivors' deceased loved ones' symptoms were not controlled and concerns existed about physician communication.

More than one third of respondents (Teno et al., 2004) whose loved ones were either in an institution or at home reported lack of emotional support for their loved one and one or more concerns with family emotional assistance. In the study, over two thirds of bereaved family members believed their loved one was not always treated with respect at the end-of-life, regardless of whether the setting was an institution or at home. Family members reported higher satisfaction and fewer needs when care was delivered by a hospice agency (Teno et al., 2004).

In an empirical study by Gade et al. (2008), the impact of an interdisciplinary palliative care team measured patient satisfaction, clinical outcomes, and cost of healthcare from over 500 patients with life-limiting illnesses in a randomized multiple center controlled trial. The interdisciplinary palliative care teams were used as the intervention and the control group was treated with usual hospital care. In this study, the interdisciplinary palliative care teams were comprised of palliative care physician, nurse, hospital social worker, and chaplain.

Results of the Gade et al. (2008) study showed patients who received interdisciplinary palliative care team intervention had greater satisfaction with their hospital care and providers, more advance directives at discharge, lower intensive care admissions if readmitted, and lower total health costs. There were no differences in 
overall survival or in physical, emotional, and spiritual area scales upon enrollment or discharge between the groups. The study's authors reported increased patient satisfaction was due to the interdisciplinary palliative care team's ability to deal with the patient's and family's desire for information and assisting them in medical care decisions. This study demonstrated evidence of an interdisciplinary palliative care teams' positive impact on satisfaction of care and decreased health costs (Gade et al., 2008).

Another empirical study of interdisciplinary palliative care team efficacy found positive patient and family outcomes. In a study of patients receiving palliative care in China, Leung et al. (2010) found that the patients who were receiving palliative care physical symptoms worsened, their psychological well-being, social support, and spirituality continued to improve. Most patients experienced a "good death" (e.g., quality of death; p. 1433) with the assistance of an interdisciplinary palliative care in the hospital (Leung et al., 2010).

Nelson et al. (2010) conducted nine focus groups comprised of 48 patients and family members to learn how they defined high-quality intensive care unit palliative care. Several main domains emerged as the participants described high-quality intensive care unit (ICU) palliative care. These were a) timely, clear, and compassionate communication with the family by clinicians, b) patients' preferences, goals, and values were the focus of clinical decision-making, c) comfort and dignity were maintained in patient care, d) families had open access and were physically located near their loved one, and e) interdisciplinary support for families was available during critical illness. Families who lost loved ones stressed the importance of bereavement care (e.g., support group 
information, follow-up phone call or letter to the family, receiving a sympathy card; Nelson et al., 2010).

Two other studies showed benefit from interdisciplinary palliative care teams. Daly, Douglas, Gunzler, and Lipson (2013) found individually tailored support services (i.e., symptom management, education, psychosocial and spiritual supports, and advance care planning) provided by an interdisciplinary palliative care team were associated with improved health-related quality of life in some patients. A quantitative study of pediatric palliative care provided by interdisciplinary team from Hays et al. (2006) found several processes to be essential for end-of-life care. These were a) patient and family preferences should be implemented in a dynamic plan of care, b) communication should be multidirectional with all clinicians and providers, and c) early involvement of insurance providers improves aspects of quality of life and family satisfaction (Hays et al., 2006).

Systematic reviews and empirical evidence suggest interdisciplinary palliative care teams improve the patient's quality of life by reducing pain, managing symptoms and improve patient and family satisfaction of care. Guidelines from the NCP and the National Quality Forum exist to ensure high-quality palliative care is available and provided; however, this does not always happen. Ferrell, Smith, Levit, and Balogh (2014) stated patient-care is often not as accessible, coordinated, patient-centered, or evidencebased as it could be.

Interdisciplinary team dynamics are complex and their interactions can be complicated. Effective interdisciplinary team functioning requires education, training, 
and resources (O’Connor \& Fisher, 2011). There appears to be some agreement that it is difficult to become an effective interdisciplinary team (Sargent et al., 2008).

\section{Effective Interdisciplinary Palliative Care Teams: Optimal Functioning}

Effective teams are dependent on communication, interpersonal relationships, structure and composition of the team, and administrative duties (Youngwerth \& Twaddle, 2011). Interdisciplinary teams are constructed of professionals from many disciplines who bring different perspectives to the team to accomplish the complex task of providing palliative care for a patient and their family. A focus on well-defined roles, leadership type, and development of competence between the professions can increase opportunities for useful collaboration among team members and result in higher quality palliative care (Klarare et al., 2013).

An optimally functioning interdisciplinary palliative care team involves outstanding training, excellent communication, description of team tasks, and responsibilities for each team member (Vissers et al., 2012). High functioning interdisciplinary teams demand effective communication within the team about patient care and treatment (Bokhour, 2006). The mere assembly of the members into a team does not presume it will be successful; effective teamwork requires effort, which has not been explicitly recognized in healthcare (Sargeant et al., 2008).

A conceptual framework to view interdisciplinary collaboration is Bronstein's two-part model (2003) of optimal interdisciplinary collaboration. Components of part one of the model are a) interdependence and flexibility, which are described as interaction between team members to achieve goals, b) newly created professional collaborative activities that make the most of each team members' skills, c) collective ownership of 
goals or the shared responsibility for goal attainment, and d) reflection on process to bring consciousness to the process of collaboration.

Bronstein (2003) defined the components of the first part of the model. Interdependence is the occurrence and reliance on interactions of each team member where each is dependent on the other team members to achieve their goals. This requires a very clear understanding of one's individual role and the other team members' roles. Interdependence is described as formal and informal time together, oral and written communications between team members, and respect from each member for other members' professional contributions.

Bronstein's (2003) component of flexibility was described as deliberate roleblurring. This was defined as altering one's professional role in order to respond creatively for what is needed at the time. Flexibility is demonstrated when an interdisciplinary palliative care team chaplain answers patients' simple questions about advance directives.

Bronstein (2003) listed newly created professional activities as acts of collaboration achieved by the team, which are more than would be possible if the disciplines acted alone. The plan of care for the patient, created by the interdisciplinary palliative care team, is one example of a newly created professional activity. It is more broad and encompassing of the patient's needs than if only one discipline had produced it.

Shared responsibility is the hallmark of collective ownership of goals component (Bronstein, 2003). The patient and family with the diverse disciplines on the team, design, define, develop, and accomplish treatment goals. Collective ownership of goals is 
illustrated when each professional shares responsibility for success and failure of attaining the patient goals. It is also demonstrated when constructive feedback, disagreements, and debates occur within the team and with the patient and family.

The Bronstein (2003) model considered the essential ingredient of interdisciplinary collaboration to reach consensus among team members, which comes from free communication, participation of diverse disciplines, and agreement on goal attainment. The final component of the part one model of interdisciplinary collaboration is reflection on process. This is time where team members reflect and discuss their working relationship in order to fortify their collaborative dealings and usefulness (Bronstein, 2003).

Wittenberg-Lyles, Parker-Oliver, Demiris, and Regehr (2010) used Bronstein's (2003) model in their qualitative study of interdisciplinary team collaboration. The Wittenberg-Lyles et al. (2010) study examined perceived and actual collaboration among interdisciplinary team members within a hospice context. Although the study uses interdisciplinary palliative care teams from a hospice, their findings may inform collaboration for interdisciplinary teams.

The study used the components of Bronstein's (2003) model of optimal interdisciplinary collaboration as a lens through which to view team collaboration. Participants in the Wittenberg-Lyles et al. (2010) study were 43 interdisciplinary palliative care team members, comprised of 17 nurses, three social workers, three chaplains, two medical directors, and 18 other team members (e.g., volunteer coordinators, bereavement coordinators, medical students, home health aides, and an executive director of the hospice), which represented two interdisciplinary palliative care 
teams (Wittenberg-Lyles, Parker-Oliver, Demiris, \& Regehr, 2010). The study also included 13 adult children caregivers, which is relevant, as the patient and family are the unit of care within the interdisciplinary team context (Saunders, 2000).

The results of Wittenberg-Lyles et al. (2010) study showed nurses had the most interdisciplinary collaborative communication (57.1\%), which was followed by medical directors (20.4\%). Non-medical staff (e.g., social workers, chaplains, others) contributed less than one fourth of all collaborative communication within the interdisciplinary team meetings. The study revealed a gap between contributions of medical disciplines (i.e., nurses and medical directors) and non-medical disciplines (i.e., social workers, chaplains, bereavement coordinators, and others) in interdisciplinary team meetings. The gap in contributions to collaboration may explain why collaboration was not present in $30 \%$ of the patient care discussions, which were analyzed for the study (Wittenberg-Lyles et al., 2010).

Wittenberg-Lyles et al.'s (2010) results determined role ambiguity (e.g., not knowing where one role ends and another begins) was the result of a lack of collaboration in team meetings and was a cause of disparity in team member contributions. The Bronstein (2003) model of role interdependence and role flexibility was expected to be used on the team as a way to achieve team goals; however, this was not the case in the study. Team members may need more understanding and education of their own role and the roles of other interdisciplinary palliative care team members in order to promote collaboration (Wittenberg-Lyles et al., 2010). The results also showed newly created professional activities (Bronstein, 2003) were more likely to occur in interdisciplinary team meetings when caregivers were present (Wittenberg-Lyles et al., 2010). 
Collaboration is fundamental to optimal interdisciplinary team function. The second part of the model by Bronstein (2003) involved four factors that influence the collaboration process. These are the professional role, structural characteristics, personal characteristics, and history of collaboration. When these factors are present, interdisciplinary energies are supported; however, when these are absent, barriers are created which weaken interdisciplinary efforts (Bronstein, 2003).

According to Bronstein (2003), each discipline must possess a strong sense of professional role. This is defined as support for each discipline's values and ethics, respect for colleagues, holistic view of practice, and a perspective that is similar or complementary to the other team members' perspectives. One must be committed to two roles; one's professional role and one's team role as an interdisciplinary collaborator (Bronstein, 2003).

The second influence within Bronstein's (2003) two-part model of optimal interdisciplinary collaboration are structural characteristics. Structural characteristics are described as a manageable number of cases for the team, institutional support for interdisciplinary collaboration, professional autonomy, and time for collaboration. These characteristics do not simply involve the team; they are required on multiple levels including individual, group, and institutional (Bronstein, 2003).

The third influence within the Bronstein (2003) model considered the personal characteristics of each member. Pertinent characteristics considered for effective collaboration were trust, respect, understanding, and positive attitudes for collaboration. The last influence within the Bronstein (2003) model on interdisciplinary collaboration 
was a history of collaboration. A history of collaboration and positive interdisciplinary experiences in internship positions support productive collaboration (Bronstein, 2003).

A qualitative study (Junger et al., 2007) of 19 members of an interdisciplinary palliative care team included interviews one year after starting a palliative care department in a hospital in Germany. The study found communication, team philosophy (e.g., team's understanding of the work to be done), work climate, and team commitment were relevant for cooperation on an interdisciplinary palliative care team (Junger et al., 2007). Both formal communication (e.g., technically necessary exchange) and informal communication (e.g., anecdotes and black humor) were necessary for smooth team functioning as reported by the team (Junger et al., 2007).

Other aspects in the Junger et al. (2007) study that were important to team functioning were clarity of goals, roles, procedures, and a friendly atmosphere, and positive working environment. The study also found that team members must be able to deal with death and dying, and this ability is crucial for cooperation on the interdisciplinary team. New teams require adequate coping options, education on expected challenges, knowledge of potential conflicts, sufficient time to discuss common goals, and maintenance of clarity of these goals, roles, and procedures (Junger et al., 2007).

Sargeant et al. (2008) used nine multiprofessional focus groups to study the characteristics of effective interprofessional teams. The 61 focus group members described experiences of interprofessional health care teams, identified characteristics of effective teams, and defined learning needs to work effectively as members of interprofessional collaborative teams. Sargeant et al. (2008) found effective team 
members understand and respect team members' professional roles, understand teams are dynamic and need ongoing work, share a common language and understanding of healthcare, possess practical knowledge for patient management among providers, and communicate well and often with team members, in informal and formal ways.

Teamwork requires knowledge, skills, and attitudes, which includes the education to develop these abilities. Findings from Sargeant et al. (2008) support understanding the roles of other team members in order to establish specific communication and team meeting skills to be essential. More findings included the importance of an attitude of respect and appreciation of others, willingness to learn new ways of working together, as well as departing from traditional medical hierarchical communication. Sargeant et al.'s (2008) results demonstrated effective teamwork is demanding and simply working with others is not enough to create a valuable team; interprofessional educational interventions help develop the knowledge, skills, and attitudes for effective teamwork.

Effective interdisciplinary palliative care teams consist of interconnected team members who collaborate and negotiate competing components within the team, and keep in mind the goal of the team, which is to provide the best care for the patient and the family members. Communication is the essential feature of helpful health care teams (Sargeant et al., 2008). Healthy and effective teams require training (Bornstein, 2003) and their valuable collaboration is crucial for the best patient care and must be systematically supported by individual, team, and organizational levels (O'Connor et al., 2006; Youngwerth \& Twaddle, 2011).

\section{Effective Interdisciplinary Palliative Care Teams: Barriers and Challenges}

Communication within the team and with the patient and family is the primary 
feature of interdisciplinary palliative care team collaboration (Bokhour, 2006; Goldsmith et al., 2010; Junger et al., 2007; Sargeant et al., 2008). However, team work is complex and barriers and challenges exist that may affect helpful interdisciplinary team interaction. Examples of barriers and challenges that negatively affect interdisciplinary palliative care team functioning are failures in communication (Goldsmith et al., 2010; Mazor et al., 2012), the lack of a common language between team disciplines (Gachoud et al., 2012; Mellor et al., 2002), limited input from nonmedical team members and biomedical dominance (Wittenberg-Lyles, Oliver, Demiris, \& Regehr, 2009; WittenbergLyles et al., 2010), existence of a traditional biomedical hierarchy (Abramson \& Mizrahi, 2003; Hartrick-Doane et al., 2012), and role flexibility and ambiguity (Bronstein, 2003; Junger et al., 2007; Klarare et al., 2013; Nancarrow et al., 2013; Wittenberg-Lyles et al., 2008).

In a qualitative study by Goldsmith et al. (2010), ethnographic observations and interviews were gathered from six interdisciplinary team participants who were each completing a yearlong geriatric and palliative care fellowship. Goldsmith et al. (2010) found each interdisciplinary team member's own professional and ethical framework was used to process the stories of dying patients and their families. Using the participants' unique points of view, this process helped team members identify instances of collaboration and the barriers to collaboration. The reflective aspect of the interview allowed each team member a greater understanding of their role in the patient's care.

Results from Goldsmith et al. (2010) showed role differences and communication highlighted by the team members' clear descriptions of lack of engagement with the case physician. A key aspect of effective interdisciplinary team care is possessing knowledge 
about the other disciplines, especially the discipline's perspective with regard to the patient's goals and plan of care. The discipline-specific knowledge each team member brought to the interdisciplinary process strengthened team collaboration, even if conflict occurred during treatment planning (Goldsmith et al., 2010).

End-of-life patients are often vulnerable and overwhelmed. If a patient or their family believes something went wrong with their care, the team members are obligated to respond. Mazor et al. (2012) conducted a qualitative study of cancer patients' care experiences, interviewing those who had experienced a breakdown in their loved ones' care. Within the Mazor et al. (2012) study, frequent breakdowns in patient cancer treatment care were examined. Patients were interviewed as to whether they believed something had gone wrong with their care that caused harm or could have been prevented. Of the 78 participants, $28 \%$ believed something had gone wrong in their care, such as a delay in diagnosis, treatment, infections, and prolonged complications from treatment. Another $47 \%$ described a communication problem that did not interfere with their medical care (e.g., difficulty with communication exchange) and $24 \%$ of the participants faced an issue with both medical care and communication problems (Mazor et al., 2012).

All of the patients in the Mazor et al. (2012) study who described a "communication breakdown" (p. 1786) with or without a breakdown in medical care, reported emotional or psychological consequences, as did those who had a failure of medical care. Over $50 \%$ of the patients in the study indicated their relationship with their clinician was broken after the event and nearly all (90\%) of the 78 participants reported making changes in their health-care related behavior because of the event. Mazor et al. 
(2012) conclude that patient perceived problematic events in cancer care may be relatively common, physically and emotionally harmful, often go unreported by the patient, and result in damaged clinical relationships. In providing patient-centered care, more consideration should be given to improved communication with patients which may prevent problems in their care (Mazor et al., 2012).

Another problematic area within the communication domain is the lack of common language for the interdisciplinary palliative care team. Mellor, Hyer, and Howe (2002) studied the lack of a common language as a challenge to interdisciplinary team function. Each discipline has its own professional language and shorthand, and often the same phrases refer to very different concepts. Mellor et al. (2002) use the term "support systems" as an illustration of discipline-specific language barriers. For example, to nurses, support systems means life support equipment, and to social workers, the term is used to describe the patient's family and friends who provide assistance (Mellor et al., 2002).

All members of the interdisciplinary team are expected to address the patient's need for spiritual care; however, the gap between a medically-focused education and a spiritually-focused education may create a potential barrier to understanding and coordination of care (Puchalski, 2012). The IOM (2008) described this barrier as likely inconsistency in medical education of health care providers to address psychosocial healthcare (e.g., including spiritual concerns) and the education received by the psychosocial health care providers who concentrate on such concerns. There is no way to assess the education or competency of medical healthcare providers in assessing or addressing psychosocial needs as a result of their education (IOM, 2008). 
Phenomenological research on the disciplinary concept of patient-centeredness within an interdisciplinary team demonstrates the challenge of finding common language concepts. Results from Gachoud, Albert, Kuper, Stroud, and Reeves (2012) revealed finding a common language and a commonly understood goal to be complex, especially between nurses and social workers on the interdisciplinary team. In this study, physicians felt they were less patient-centered than other professional groups, nurses and social workers considered themselves to be more patient-centered than others (Gachoud et al., 2012); however, this finding produced no evidence of friction between the professions.

Limited input from nonmedical team members in group meetings and the resulting biomedical information dominance are a challenge to interdisciplinary collaboration. In their study of four interdisciplinary teams' meeting communication dynamics within the hospice context, Wittenberg-Lyles, Oliver, Demiris, Baldwin, and Regehr (2009) found biomedical information to be the most commonly shared material. Nurses obtained communication control in interdisciplinary team meetings primarily because they are reporters of patient biomedical information and they see patients more often than other members of the team.

In their study, non-medical disciplines (e.g., social workers and chaplains) did not share equally about aspects of patient care and the patient's psychosocial or spiritual well-being was reduced as a result of the primary reporting by the nurse (WittenbergLyles et al., 2009). The structure of nurse as primary reporter in the interdisciplinary meetings is a barrier that inhibits other disciplines from sharing information for discussion about all aspects of the patient's care (Wittenberg-Lyles et al., 2009). Because the nurse's report creates a pattern of reporting and reviewing patient cases, it is difficult 
for new information sharing to occur within the established arrangement (WittenbergLyles et al., 2009). Meier and Beresford (2008) stated there has been a "historical imbalance in power and responsibility" (p. 679) between medicine and psychosocial and spiritual team members; and while palliative care teams support equality of team professionals, each team requires well-defined ways of reporting information.

The existence of the traditional biomedical hierarchy is another barrier to interdisciplinary palliative care team collaboration. Traditionally, the physician has been the leader of medical teams, with others in supporting roles (Abramson \& Mizrahi, 2003; O'Connor et al., 2006); however, this is not the model for interdisciplinary palliative care teams. The physician is accountable for the patient's medical treatment, but the leadership of the interdisciplinary palliative care team should vary from case to case, as this is dependent upon the needs of the patient and family (Cummings, 1998).

Power that is established through the typical top down medical model can be difficult for members of other disciplines who are accustomed to equality and democratic power relations (O'Connor et al., 2006). The power and authority of the biomedical knowledge hierarchy with regard to communication have a negative effect on positive team operations (Hartrick-Doane et al., 2012). Collaboration occurs when hierarchy is not present and all team members' knowledge is appreciated and considered within the treatment goals (Fewster-Thuente \& Velsor-Friedrich, 2008).

Klarare et al. (2013) reported medical dominance on the teams can cause an imbalance within the stated palliative care mission, which is to provide comprehensive interdisciplinary care to the patient and family. A historical difference between power and responsibility exists between physicians and psychosocial-spiritual professionals that 
needs clear leadership and reporting structures, as well as training in hospital cultures (Meier \& Beresford, 2008). Traditional power imbalances, combination of disciplines, required team member attributes and skills, as well as the unique profile of every patient cause optimal interdisciplinary team work to be complex.

Role flexibility, role ambiguity, and role conflict are challenges for the professionals on an interdisciplinary team. Each member of an interdisciplinary palliative care team is expected to have a certain amount of role flexibility, or ability to step into another's role on the team, which promotes interdependence (Bronstein, 2003). However, when professional boundaries and responsibilities are challenged, patient care can be hampered (Klarare et al., 2013).

Role ambiguity and role conflict indicated ineffective cooperation in a palliative care team (Junger et al., 2007). Role ambiguity (e.g., lack of role clarity) can occur during role flexibility, and it is most likely to occur to the psychosocial and spiritual members of the team. The concept of psychosocial care blends emotional, social, and spiritual support and is an area of patient care that is shared by the interdisciplinary team. All psychosocial care is shared by the team; moreover, psychosocial care is a contested area of patient care that social workers, palliative care nurses, chaplains, counselors, and psychologists want to claim (O'Connor et al., 2006), although counselors are often not part of these teams.

In a qualitative study designed to explore interdisciplinary palliative care teams' delivery of psychosocial care, O'Connor and Fisher (2011) interviewed seven palliative care team members. Two themes emerged from their research, which were the "Lack of clear role boundaries" and "Strategies for maintenance of role boundaries" (p. 193). The 
lack of clear role boundaries was most problematic for the psychosocial team members, as the non-psychosocial participants suggested any team member can provide support for these issues. As a result, the lack of clear role boundaries in the psychosocial area led psychosocial professionals to assert specific expertise in order to maintain their role on the team (O'Connor \& Fisher, 2011).

O'Connor and Fisher (2011) also determined another way to protect one's role is to minimize the importance of other professions. In their study, this was demonstrated by a split between professionals who had training in psychological care and those who did not have this training. The authors argue that as a result of unclear role boundaries, psychosocial team members struggle to be seen as rightful members of the interdisciplinary palliative care team within the domain of patient care ( $\mathrm{O}^{\prime} \mathrm{Connor} \&$ Fisher, 2011). Oftentimes, when a team member's boundary, or territory has been violated, total team collaboration is inhibited (Klarare et al., 2013).

Research demonstrates some disciplines experience more role confusion on the interdisciplinary palliative care team than others. In survey research from chaplains who participate in interdisciplinary palliative care teams, Wittenberg-Lyles et al. (2008) found the chaplain's professional role to be unclear on the interdisciplinary team because other team members did not understand it. Chaplains experience role conflict with social workers, but receive the most support from social workers (Wittenberg-Lyles et al., 2008).

Chaplains help to promote interdisciplinary collaboration within the team by supplying personalized self-care, giving daily encouragement, and acting as the team conflict manager. They have unique roles that extend beyond their patient and this is not 
a role that is expected of the other disciplines. Chaplains have a professional role to fill in the interdisciplinary collaborative process not only for patients and families, but also for team members (Wittenberg-Lyles et al., 2008).

Research has established multiple barriers and challenges that prevent interdisciplinary palliative care team effectiveness. Communication failures, the lack of a common language between diverse disciplines, biomedical information dominance that causes limited sharing from nonmedical team members, existence of biomedical hierarchy, and issues with role flexibility each have an effect interdisciplinary palliative team collaboration. When collaboration is lacking, patient care may suffer because all domains are not represented in the treatment plan. The evolution of an interdisciplinary team takes time as the professionals grow into their roles and learn how to work together; however, the process can be "conscious and planned, or unconscious and accidental" (Remke \& Schermer, 2012, p. 291).

\section{Interdisciplinary Palliative Care Teams: Emotional and Behavioral Influences}

Participation on an interdisciplinary palliative care team has effects on its team members. Saunders (2000) described the care of the dying as a "sometimes overwhelming experience," (p. 9) which can be painful and confusing to all staff (Saunders, 1978). Members of interdisciplinary teams work in environments that are challenging because they meet death every day. Junger et al. (2007) determined interdisciplinary team members must have the ability to cope with death and dying of patients, their own bereavement, as well as provide support to grieving family members. Webster and Kristjanson (2002) studied the experiences of six interdisciplinary palliative care team members who have continuously worked for a minimum of five 
years. Their study gives insight into the reasons team members chose to work in palliative care, stages they went through over time, and what has kept them working in the field. Webster and Kristjanson (2002) found a common work trajectory that consisted of five phases of experience among the six participants: The Awakening, Making the Connection, Committing to the Philosophy, Reaping the Rewards, and Soldiering On.

Webster and Kristjanson (2002) described The Awakening phase as a period of developing awareness the palliative care workers gain as a result of the difficulties associated with caring for the terminally ill, especially that their needs were different than caring for others who were ill. The next phase, Making the Connection, was recognized as making a connection with the concepts of palliative care. Although the connections were different for the participants, all reported a personal aspect (e.g., losses of their own) that had an effect on them and inspired their commitment to the field.

The third phase of experience found by Webster and Kristjanson (2002) was called Committing to the Philosophy. This was the time when the interdisciplinary palliative care team became very important to the participants. Team members stated they had feelings of personal growth, enjoyment, and shared commitment of working with a great team, committing to the philosophy of palliative care, and supporting the multidisciplinary approach to care of the patient (Webster \& Kristjanson, 2002).

Webster and Kristjanson (2002) found Reaping the Rewards to be a phase where all participants reported satisfaction in caring for the patient and the family, including cumulative rewards of achievement, growth, and a sense of privilege providing palliative care. This was the phase when the positive consequences outweighed the difficulty or sadness of working with the terminally ill. One participant stated, "It's sad sometimes, 
but actually it's much more rewarding than anything else I've ever done" (Webster \& Kristjanson, 2002; p. 871).

The last phase in the work trajectory for palliative care team members found by Webster and Kristjanson (2002) was called Soldiering On. Changes were happening (e.g., decline in funding and support for palliative care), which were reported as challenging. However, the participants remained in palliative care because they felt their work was a privilege, gave them a sense of achievement, worth, and energy. Another theme that kept them in palliative care was working on an interdisciplinary team; this was described as providing companionship, mutual respect, and support (Webster \& Kristjanson, 2002).

The study by Webster and Kristjanson (2002) demonstrated the role of colleagues and the value of the interdisciplinary team approach to be helpful with the stress of doing palliative care work. The participants' stories of time spent on an interdisciplinary palliative care team suggest they are able to balance the sadness and satisfaction of their work over time. The authors stressed the importance of the interdisciplinary palliative care team as a system of support for its members (Webster \& Kristjanson, 2002).

Interdisciplinary team members' spirituality (i.e., sense of life's meaning, the supreme-being, nature) may be affected by working on a palliative care team. The interaction of interdisciplinary team members' spirituality and their professional work is an area which could influence how they work with patients. Mitchell (2011) suggested one way to effectively work with palliative care patients and their families is for the interdisciplinary team member to contemplate their own beliefs on the end-of-life and death. 
Clark et al. (2007) studied the prevalence of spirituality among hospice interdisciplinary team members and to determine if spirituality had an effect on job satisfaction among the team members. A premise for the Clark et al. (2007) study was the belief that interdisciplinary palliative care team members are more spiritual when compared to other work settings because team members deal with serious illness, the dying process, and other end-of-life issues, in addition to providing support for the family of the patient. This research surveyed 215 interdisciplinary palliative care team members (e.g., nurses, home health aides, social workers, chaplains, physicians, and others) from the hospice context. The participants completed three scale measures related to spiritual well-being, spirituality integration, and job satisfaction (Clark et al., 2007).

Clark et al. (2007) found a "remarkably high prevalence of spirituality among this interdisciplinary team" (p. 1326) from the 215 interdisciplinary palliative team members. Results from their hospice-focused empirical study found interdisciplinary palliative care team members integrated their spirituality at work. They also had greater selfactualization, which significantly improved their job satisfaction. Few studies have empirically examined spirituality and job satisfaction within an interdisciplinary palliative care team. The study from Clark et al. (2007) may provide information that pertains to palliative care.

Sinclair, Raffin, Pereira, and Guebert (2006) conducted a qualitative study of collective spirituality of 20 interdisciplinary palliative care team members. From participant interviews and focus groups, Sinclair et al. (2006) found the culture within palliative care promoted spiritual contemplation among the interdisciplinary team members, as individuals and as a team. Within their sample, participants believed 
palliative care was considered to be a calling and palpable spirituality could be felt on the hospital unit. A collective spirituality was identified and this related to wholeness, meaning, integrity, and personal journeying on the individual and the team level (Sinclair, Raffin, Pereira, \& Guebert, 2006).

\section{Interdisciplinary Palliative Care Teams: Education}

The integration of interdisciplinary teamwork into professional healthcare education is vital for high quality palliative care (Youngwerth \& Twaddle, 2011). Interdisciplinary education, which includes clinical placements for experiences with the dying as part of an interdisciplinary palliative care team, is essential for patient-centered practice of future health care professionals (Head et al., 2012), including counselors. Ideally, interns are placed with professionals in their fields on interdisciplinary palliative care teams who are knowledgeable and respectful about all team disciplines and roles, possess excellent collaboration and communication skills, have clear team leadership, acquire training on team dynamics and, according to the NCP Standards (2013), also be certified in hospice and palliative care, when this certification is available.

Interdisciplinary palliative care teams who are high functioning will be in the best position to give the interns experiences so they may learn how to become effective team members.

International and national agencies recommend interprofessional education (i.e., education which involves educators and learners from two or more health professions) to prepare professionals to provide palliative care. The mandate from the WHO considers interprofessional education as necessary for a collaborative practice-ready health workforce (WHO, 2010). The WHO defines a collaborative practice-ready health worker 
as one who has learned how to work on an interdisciplinary team and is competent to do so (WHO, 2010).

The IOM (2014) stated in its most recent report that special practice in hospice and palliative care in the education of health professionals has greatly improved; however, problems still remain. These are a) insufficient attention to medical and nursing school curricula; b) educational silos exist which inhibit the development of interprofessional teams; and c) deficits in preparing physicians with appropriate communication abilities. System support and quality standards are required to encourage improved clinician communication competencies and more frequent and constructive clinician-patient discussions. Broad improvements within palliative care education of professionals prepares for the delivery of sustainable end-of-life care for patients and families (IOM, 2014).

\section{Interdisciplinary Palliative Care Team: Need for Study}

The population of people age 65 and older is projected to grow significantly, to 72 million, representing nearly $20 \%$ of the total U. S. population by 2030 (Federal Interagency Forum on Aging-Related Statistics, 2012). Society will be significantly affected by the projected growth of this segment of our population. This large group will impact the ways families and healthcare providers care for them. Medical care choices, caregiving, changes in living arrangements, financial worries, and decisions about the end-of-life are all issues that will confront this older age group of Americans.

Today's patients often face many issues as they cope with life-threatening illness. The patient's family who provides care, adapts to the loved one's illness and eventual death, cannot be responsible for all aspects of patient care (Cummings, 1998). 
Interdisciplinary teams bring together multiple disciplines whose common goal is to improve the quality of life for the palliative patient. Comprehensive palliative care includes an interdisciplinary effort to alleviate the physical, psychosocial, and spiritual concerns of the patient and the family (Bakitas et al., 2009).

Providing team care is complex and requires trained professionals who work together to provide seamless, high-quality, patient-centered, family-oriented, completely accessible care for those and their family members at the end-of-life (IOM, 2014). O'Connor and Fisher (2011) found efficacious team functioning does not happen without effort; it requires education and training, as well as organizational structures and resources to support it. Interdisciplinary teams require work to become effective and this necessary work is not typically recognized by the healthcare profession (Sargent et al., 2008). One way to prepare future generations of health professionals for collaboration is through interprofessional education (WHO, 2010). Empirical studies, supported by theoretical constructs, may help determine if team members-in-training are learning how to be useful and contributing team members as a result of interprofessional education experiences.

\section{Interdisciplinary Palliative Care Team: Core Domains and Disciplines}

Interdisciplinary teams are the foundation of palliative care throughout the world (Hurley et al., 2014). Medicare-certified agencies (i.e., hospices) by law must provide each patient with a full interdisciplinary team, consisting of a physician, nurse, social worker, chaplain, and volunteer (Medicare and Medicaid Programs: Hospice Conditions of Participation, 2008). However, missing from this list are other important disciplines, 
such as those within the counseling profession, such as clinical mental health counselors, counselors, and licensed professional counselors.

Interdisciplinary palliative care teams in other situations (i.e., all non-hospice

palliative care) are not bound by the conditions of federal programs. These teams are not required to meet those conditions for robust interdisciplinary team composition. Interdisciplinary care is a core value in palliative care, a foundation of quality practice, and an essential part of team-based palliative care practice, however, its form can vary due to available resources and local preferences (Ogleby \& Goldstein, 2014).

\section{Core Discipline: The Patient and Family}

The patient and the patient's family are considered to be the unit of care, which is one of the core principles of palliative care (Saunders, 2000). Palliative care offers a unique approach by integrating the needs of the family into the care of the patient (Gelfman, Meier, \& Morrison, 2008). The interdisciplinary palliative care team considers the patient and the patient's family to be the unit of care, as well as part of the team, and the patient and family are essential to help develop the goals of care (Cummings, 1998; Wiecha \& Pollard, 2004).

Family caregivers are crucial to the patient's care and different settings of patient care (e.g., in the home or inpatient palliative care unit) create different roles for family caregivers (Hurley et al., 2014). In their qualitative research, Goldsmith et al. (2010) found family members and caregivers are frequently the focus of work for a team because they make decisions for the dying patient. Families are constantly trying to maintain balance as they adjust to the extensive changes from life-threatening illness (Doka, 2009). 


\section{Core Discipline: Medicine and the Physician's Role}

The physician has a central role on the interdisciplinary palliative care team because relief of pain and other distressing physical symptoms are the most important aspect of palliative care (Cummings, 1998). The goal of pain and symptom management is the foundation for promotion of psychological, social, and spiritual quality of life (NCP, 2013). Primary palliative care skills as provided by the physician include basic management of pain and symptoms, basic management of depression and anxiety, and basic discussions about prognosis, goals of treatment, suffering, and code status (i.e., choices about resuscitation; Connor, 2009; Quill \& Abernethy, 2013).

Primary care physicians may deliver core elements of palliative care, such as providing basic symptom management and aligning treatment with a patient's goals (Quill \& Abernethy, 2013). However, complex palliative care is often directed by a palliative medicine specialist. A palliative medicine specialist is a formal medical specialty that has grown out of end-of-life care into an expanded role, which provides palliative care in earlier stages of disease (Quill \& Abernethy, 2013). Palliative medicine specialists possess other skills, such as expertise in communication, complex decision making, symptom management (i.e., pain control), psychosocial care, care of the dying, and transition of care (Bruera \& Hui, 2010; Davis, Walsh, LeGrand, \& Lagman, 2002).

Skills required of the palliative medicine specialist are more complex than those of primary palliative care. These skills are management of treatment resistant pain, management of more complicated depression, anxiety, grief, and existential distress, assistance with conflict resolution of goals or treatment methods within the family, between staff and families, and among the treatment team as well as direct cases of near 
futility (Quill \& Abernethy, 2013). Medical futility refers to the physician's prediction that as a consequence of irreversible illness or injury, further therapy will not improve the patient's condition, and as a result, should not be attempted (Bernat, 2005).

Examples of medical futility include brain death, persistent vegetative state, and advanced Alzheimer's disease. In cases such as these, a power struggle may result over continued treatment between families and physicians (Bernat, 2005). Physicians' clinical and human skills are tried in working with patients who are at the end-of-life and being a member of an interdisciplinary palliative care team provides an opportunity to learn about one's self, the power, and the limitations of medicine (Quill, 2001).

\section{Core Discipline: Nursing and the Nurse's Role}

Using evidence-based interventions, and by their presence, palliative care nurses make assessments, diagnoses, and perform interventions in patients with life-limiting illness, including their families, to accomplish constructive patient results that may increase quality of life and relieve suffering (Lynch, Dahlin, Hultman, \& Coakley, 2011). Ideally, nurses provide a holistic approach to care, pain and symptom management, cultural competence, effective communication abilities, recognition of ethical and legal aspects of care, and knowledge of how to care for the imminently dying (Sherman, 2010). The interdisciplinary team member who has the greatest amount of contact with the patient and family typically is the nurse (Cummings, 1998).

Nurses help the patient with the advancing disease and its accompanying distressing symptoms, providing care for patients who undergo the complex physical, emotional, and spiritual wants of those at the end-of-life, so they may die with comfort and dignity (Wu \& Volker, 2012). Nurses are often case managers in palliative care, 
which means they are responsible for disciplinary coordination of care and are the link to the physician to see all orders for care are achieved (Connor, 2009; Nelson et al., 2011). Nurses have a key role in the assessment and management of pain; however, they are vital to the palliative team process to establish the patient's goals of care (Nelson et al., 2011).

Refractory (i.e., uncontrollable) pain is common in palliative patients, so controlling pain is of upmost importance (Bookbinder \& McHugh, 2010). In an empirical study of predictors of quality of life in cancer patients, the nurse, as member of an inpatient interdisciplinary palliative care team, needs to detect and manage physical (i.e., pain and fatigue) and psychological (i.e., anxiety and depression) symptoms, and inspire the patient to have a greater sense of control over their life-limiting condition (Peters \& Sellick, 2006). Nurses play an essential role on the interdisciplinary palliative care team for symptom management, communication, and patient-family decision making and only an integrated team of competent professionals working within effective procedures may high-quality palliative care be provided to those at the end-of-life (Nelson et al., 2011).

\section{Core Discipline: Chaplaincy and the Chaplain's Role}

Spiritual care is listed as a core domain of quality interdisciplinary palliative care by the NCP (2013). The chaplain's role is to take primary responsibility for providing spiritual care to patients, family, friends, as well as to colleagues who are part of the interdisciplinary team (Harper \& Rudnick, 2010). Spirituality is a crucial element of wellbeing and spiritual issues of hope, forgiveness, love, and reconciliation to a higher power and to others; it may surface and cause distress as patients near death (Harper \& Rudnick, 2010; Puchalski, 2007; Puchalski, 2010). 
Puchalski (2012) viewed spirituality as a fundamental human component and also recognized it as an essential dimension in a patient's health and well-being, which needs to be integrated into the treatment plan of the dying patient's care. Patients at the end-oflife deal with the largest spiritual matters of meaning, personal worth, and connection; chaplains support them as they work through these issues (Ford \& Tartaglia, 2006). The chaplain's responsibility is to incorporate the patient's spiritual needs and their expression into the plan of care. Chaplains work with patients and families to feel secure in implementing healthcare goals that reflect their core values (Flannelly et al., 2012).

Chaplains respond to spiritual distress by being present with the patient, assessing for spiritual pain, and giving spiritual care planning (Millspaugh, 2005). When chaplains provided empathetic listening, patient trust and rapport were enhanced, which led to better care and more helpful coping (Handzo et al., 2008). Unlike members of the team, the chaplain does not try to cure disease, but serves as a witness to suffering and mirrors unconditional love to the patient (Puchalski, Lunsford, Harris, \& Miller, 2006).

Chaplains who are members of interdisciplinary palliative care teams are different from community clergy who pastor churches, synagogues, or mosques. Chaplains are often ordained clergy; however, they need professional training (Harper \& Rudnick, 2010) to prepare to care for patients and their families who come from widely varying faith traditions, including those who may possess no faith at all. The chaplain is the symbol of mystery, transcendence, higher power, and tradition of religious ritual, who shows "universal compassion of shared humanity" (Ford \& Tartaglia, 2006, p. 677). A study by Cadge, Calle, and Dillinger (2011) found that chaplains consider themselves to have several roles. Chaplains see themselves as providers of traditional 
support to patients and families by comforting and performing rituals for patients;

however, chaplains also view themselves as offering a perspective of wholeness and healing to the patient-family (Cadge, Calle, \& Dillinger, 2011). They also see themselves as one who improves family and team communication (Fitchett et al., 2011).

Chaplains provide spiritual care for the patient's family and friends. Harper and Rudnick (2010) described what the chaplain does for the family system. A very important responsibility of the chaplain is to cultivate trust with the patient and family. Chaplains are present, and actively and empathetically listen to the stories from family and friends; after this group receives spiritual care from the chaplain, they may return spiritual support to the patient (Harper \& Rudnick, 2010).

In addition to serving their patients and families, chaplains are expected to supply pastoral support to staff, which includes their interdisciplinary team. Common Standards for Professional Chaplaincy describe what it means to provide pastoral care (Common Standards for Professional Chaplaincy, 2006). Chaplains are charged by these standards to provide, among others, effective pastoral support, which includes providing religious and spiritual resources appropriate to the care of patients and their families, and as well to staff.

Team members ask for chaplain support with their own spiritual care and chaplains report providing this support to team members; however, while this is expected of chaplains (Harper \& Rudnick, 2010), this support may limit the amount of spiritual care available for patients and their families (Wittenberg-Lyles et al., 2008). Chaplains also report opening interdisciplinary team meetings with prayer and serving as the team's conflict manager (Wittenberg-Lyles et al., 2008). Because chaplains also fill informal 
roles on the interdisciplinary team, (e.g., giving individual self-care and encouragement) their roles are different from the other team members and these roles compete for patient spiritual care (Wittenberg-Lyles et al., 2008).

The chaplain's interdisciplinary team role is to provide spiritual support to the patient and staff as well as bereavement care to survivors (Harper \& Rudnick, 2010; NCP, 2013). Chaplain duties also differ from other team members because they go beyond routine patient visits and regular interdisciplinary palliative care team meetings; such as regular communication with outside clergy and participation in funeral preparations (Wittenberg-Lyles et al., 2008). Additionally, chaplains are expected to help patients make difficult decisions about treatments and discuss matters pertaining to endof-life (Flannelly et al., 2012).

The strain of constant exposure to death and dying plus conflicts within the interdisciplinary palliative care team cause stress on the members (Junger et al., 2007) and the chaplain acts informally as the team's conflict manager and daily support for the team members (Wittenberg-Lyles et al., 2008). The other disciplines (i.e., medicine, nursing, social work) are not expected to fulfill such informal roles for the interdisciplinary team. Sinclair et al. (2006) report non-chaplain interdisciplinary team members found providing spiritual care to be a daunting task and they experienced role ambiguity (i.e., uncertainty where their role ended and chaplain's role began) when giving spiritual support to patients and families.

\section{Core Discipline: Social Work and the Social Worker's Role}

Social workers are an integral and appropriate part of providing good palliative care by delivering assessment and intervention within the social and psychological 
wellbeing of the patient, family, friends, including caregivers, before and after death (Monroe, 2010). They work with clients who face life-limiting situations of illnesses, either acute or long-term, coping with trauma or suicide, dying, death, and bereavement, with people of all ages, cultures, socioeconomic status, and families, both traditional and nontraditional (National Association of Social Workers [NASW], 2004). The National Association of Social Workers created standards for the practice of social work in Palliative \& End-of-life Care (NASW, 2004) and Cultural Competence in Social Work Practices (NASW, 2007). These standards inform and guide social workers as members of interdisciplinary palliative care teams.

Social and cultural competence are listed as core domains of palliative care in the NCP (2013) and these competencies are operationalized to advise social workers as they deliver end-of-life care (NASW, 2008). Social workers are advocates for the patient and their family. These clinicians receive training in cultural competence, which provides an understanding of race, ethnicity, class, sex, gender, sexual orientation, religion, marital status, age, disability, and family structure (Wesley, Tunney, \& Duncan, 2004); however, all clinicians are expected to be culturally competent providers (NCP, 2013). Social workers are aware of the "person-in-environment" (p. 298) and provide contextual understanding of the patient's complex symptoms for the palliative care treatment plan (Bornstein, 2003).

Blacker and Deveau (2010) defined the palliative care social worker as one who is able to deal more intensively with the psychosocial needs that occur with life-limiting illness. The authors put forth the following areas of focus for the palliative care social worker. These areas are a) engaging in team discussions with the patient and family 
concerning disease progression and goals for care, b) initiating discussions about advance directives and planning, and c) providing individual and family education to plan for anticipated caregiving needs for the patient and other dependents. The palliative care social worker also provides grief counseling for the patient and family for grief and loss, facilitates referrals to hospice providers, if needed, outside the hospital, conducts bereavement screening, and provides bereavement services (i.e., follow-up calls, group interventions, individual and family grief counseling). They also pursue and take part in ethics consultations (Blacker \& Deveau, 2010).

For effective practice with patients who are at the end-of-life, social workers must also possess knowledge about medical and social systems that are often barriers to those who need attention and be able to comprehensively assess the patient-family to integrate biopsychosocial and spiritual aspects into the team's overall palliative care plan of care for the patient (NASW, 2004). Social workers also manage complex patient-family issues, such as substance abuse, legal problems, and family concerns, which can have an impact on the interdisciplinary palliative care team (Klarare et al., 2013). Bornstein (2003) states the social worker on the interdisciplinary team takes on an "endless number" of roles (p. 302).

Social workers are striving to be valid and recognized members of the interdisciplinary palliative care team; however, within-team tension may result from frustration with unclear role boundaries from the psychosocial domain (O'Connor \& Fisher, 2011). Bornstein (2003) explained the social worker's interdisciplinary team role must include a strong sense of their professional role and flexibility within that role, because effective interdisciplinary collaboration demands both. Some authors view the 
social worker's professional role as flexible and easily blurred into other areas of the interdisciplinary palliative team. Puchalski, Lunsford, Harris, and Miller (2006) suggested the flexibility, openness, and the compassionate presence of trained social workers who conduct psychosocial assessments to be well suited to listen to the patient's deeper spiritual issues.

The growing role of social worker often includes helping palliative care patients with spiritual difficulties at the end-of-life; however, social workers are reluctant to intervene because they do not believe they have the knowledge to provide spiritual care (Wesley et al., 2004). Lack of available clergy is one of the reasons social workers find themselves in the position to provide spiritual care to palliative patients (Wesley et al., 2004) and a very good explanation for social workers to receive spiritual training (Fife, 2002). Patients report their spiritual requests can be met by nurses and social workers, not only by chaplains (Edwards et al., 2010).

\section{Interdisciplinary Palliative Care Team: Counseling and the Counselor's Role}

As previously mentioned, O’Connor et al. (2006) described difficulties within the interdisciplinary team and the provision of psychosocial care. The authors suggested holistic care given to the patient by the team includes psychosocial support of the patient, which each member of the team is expected to deliver. Psychological care combines emotional, supportive, and spiritual care by the team and no one discipline can dominate this area because all members of the team are to provide it by virtue of role flexibility.

The psychosocial realm of the interdisciplinary team is contested by social workers, psychologists, palliative care nurses, counselors, chaplains, and others (O’Connor et al., 2006). Counselors have the skills and knowledge to provide end-of-life 
care but few are currently members of palliative care interdisciplinary teams. The IOM (2008) and the NCP, Second Edition, recognizes counselors as an appropriate profession for interdisciplinary team membership (NCP, 2009).

Although most interdisciplinary teams do not include counselors, one large tertiary hospital in the southeastern U. S. has created a new approach to hospital palliative care service using counselors. This unique framework uses counselors to make initial contact with the family and enhance communication between the family and medical personnel throughout the patient's hospital stay. This program has the counselor at the center of the interdisciplinary palliative care team and has proven to be an effective method of managing very complex hospital palliation cases (Babcock \& Robinson, 2011) and remains an ongoing program (C. Babcock, personal communication, November 10, 2014).

One empirical study from this counselor-based palliative care program researched the influence of race on end-of-life choices (Benton et al., 2015). A retrospective study of 2,843 White and African American patients' treatment choices before and after receiving a standardized counselor-based palliative care consultation were reviewed. Benton et al. (2015) report of the $72.8 \%$ of patients who originally chose full code (e.g., use of all appropriate modalities, without restrictions, for life-sustaining treatment), only $17.6 \%$ kept this treatment choice after the palliative consultation. The Do Not Resuscitate (DNR) treatment nearly doubled, from $22.6 \%$ to $39.1 \%$, demonstrating patients who originally desired all possible resuscitation treatments, after palliative care consultation, now wanted only DNR orders as treatment. Of the patients who originally chose care and comfort only (CCO) measures, $4.6 \%$ increased to $43.3 \%$ after the palliative care 
consultation (Benton et al., 2015).

Race proved to be an important factor in treatment choices, with both groups (i.e., White patients and African American patients) moving from more aggressive treatments to less aggressive treatments after palliative care consultation (Benton et al., 2015). The percentage of African American patients who chose full code dropped from $78.8 \%$ to $22.2 \%$, and on the same measure, White patients dropped from $67.3 \%$ to $12.2 \%$ after the palliative consultation. These findings suggest the comprehensive counselor-based palliative care consultation for hospitalized patients was correlated with both White and African American patients choosing end-of-life care choices with fewer life-sustaining interventions (Benton et al., 2015).

\section{Existential Theory and Palliative Care}

The current study is situated in an existential approach that examines the communication and interaction of chaplain interns and social work interns as they participate on an interdisciplinary palliative care team clinical placement. Existential matters are prominent in palliative care. The paradoxical nature of human existence with themes of life and death, relation and isolation, hope and despair, meaning and meaninglessness infiltrate end-of-life work (Breitbart, Gibson, Poppito, \& Berg, 2004).

The existential approach emphasizes how humans find meaning in life's paradoxes coupled with our unique ability to take responsibility for our own existence (Breitbart et al., 2004). Existential thinking is well-matched to end-of-life care because it asks deep questions about the nature of humans and the nature of despair, grief, loneliness, anxiety, isolation, and anomie (i.e., personal unrest or alienation; May \& Yalom, 1995). As patients explore their life experiences, especially their negative 
emotions of anxiety and guilt, emotional and spiritual well-being may be achieved as one's life is integrated into a meaningful whole (Breitbart et al., 2004). The most frequently asked questions of hospital chaplains by palliative care patients were generally existential in nature and were about the meaning of life, death and dying, and relationships (Strang \& Strang, 2002).

An existential approach is an appropriate framework for the work of the interdisciplinary palliative care team because as patients approach death, they often focus on what is unique, precious, or problematic in their existence (Tomer \& Eliason, 2008). Frankl (2006) described man's primary motivational force as the struggle to discover meaning in life, which emphasizes spirituality (Orbach, 2008). Frankl (2006) called his existential doctrine "Logotherapy."

Within the Logotherapy approach, three values exist from which one may discover meaning in life: the creative, the experiential, and the attitudinal (Frankl, 2006; Orbach, 2008). Of special significance to the dying is their experience and attitude toward unavoidable suffering. Frankl (2006) believed life had meaning under all situations, even in hopeless conditions, up to the very end of one's life.

Others have studied and written about Frankl's existential Logotherapy and meaning-making. Existentialists May and Yalom (1995) identify death as the "ultimate concern" (p. 281). Death is considered to be a "boundary situation," that is, an urgent experience that pushes one into conflict with an existential condition. One's personal death is the most powerful human boundary situation.

When one is aware of the finiteness of life, a profound swing in perspective may happen which results in personal change. Often terminally ill patients express grief over 
personal growth gained as a result of their illness and impending death (May \& Yalom, 1995). The threat of death may deepen one's spirituality and also transform the way one sees oneself as well as the way one sees death (Tomer \& Eliason, 2008).

Tomer and Eliason (2008) proposed an existential model of death anxiety that includes death salience, belief systems about the self and the world, and three precursors: past-related regret, future-related regret, and the meaningless of death. Death salience is the degree to which one contemplates their own mortality and death. The intensity of death salience is related to one's exposure to death, either their own or other's deaths in their lives, which are affected by the importance of the relationship, amount of emotional attachment, and the newness of the loss. One's self-beliefs involve self-esteem, control, and insight about integrity (Tomer \& Eliason, 2008).

World belief systems, in the Tomer and Eliason (2008) model of death anxiety, included whether or not life is fair and honest. The three types of regret are defined as emotion related to the past (e.g., errors of commission or errors of omission), the future (e.g., action in the future is impossible), and the meaningfulness of death regards how one views death, as making sense or meaningless. Higher death anxiety is experienced if past regret and future regret are unresolved, or if death is recognized as meaningless.

This model of death anxiety was empirically studied by measuring life regrets and death attitudes in college students by Tomer and Eliason (2005). Their results found pastrelated regret and future-related regret independently predicted avoidance of death. Belief systems about the self were measured; negative affect and internal locus of control lead to an increase in future-related regret and a belief in random control and an absence of selfesteem were associated with past-related regret. This model of death anxiety provided an 
empirically validated existential conceptual format for several proven variables that contribute to the fear of death (Tomer \& Eliason, 2005).

Death is a boundary situation not only for the patient and family, but also for the interdisciplinary palliative care team. The patient, family, and team will each experience the patient's death in a different way; the patient will no longer be alive, the family will grieve the loss of their loved one, and the interdisciplinary palliative team members will have lost another patient. Professionally, the interdisciplinary palliative care team members encounter death daily and have the opportunity to promote and witness their patients' existential explorations (Breitbart et al., 2004).

The interdisciplinary palliative care team will be well served to have considered existential and transcendent issues as they work with the dying. Sinclair's (2011) ethnographic inquiry sought to produce detailed understanding of the impact of death and dying on 24 clinicians who provided end-of-life care. Analysis of semi-structured interviews and participant observation discovered three overarching categories (e.g., past, present, future) and 11 themes from the data.

Within Sinclair's (2011) study, the category of Past: The prominence of death in the early years, all participants cited early experiences with death and dying that motivated their professional practice with the terminally ill. The second category was Present: The power of death over life. The participants reported working with the dying had informed how they currently lived their lives, by living in the present, developing a spiritual life, and by reflecting on how life goes on after death. 
Finally, the last category to emerge from Sinclair's (2011) analysis was Future: The continuity of life and death. Most of the participants revealed they had reflected on their own death and imagined their own dying through the deaths of their patients. They believed facing one's mortality to be helpful and possibly a requirement to providing effective care to the dying. This study showed life-affirming lessons learned by clinicians from those who are nearing death (Sinclair, 2011).

An existential theoretical lens provides a structural view of the dying patient's concerns and reasons for making meaning of their death. As long as one is alive, meaning in life can be found (Frankl, 2006). An existential approach and the model of death anxiety (Tomer \& Eliason, 2005) provided a meaningful and logical theoretical underpinning for the end-of-life journey of the patient and for those who journey with them; their family and their interdisciplinary palliative care team. Awareness of existential factors (i.e., boundary situations) and difficulties with life regrets of the dying may help the team provide appropriate care of the patient and family.

Not only can the patient experience transcendence as they make meaning of the time that is left, the interdisciplinary palliative care team may also be inspired as they work with the dying. According to a qualitative study by Sinclair et al. (2006), transcendence was reported by the interdisciplinary palliative care team members as a mystical experience of spirituality of connection to God, or the Universe. One of the most important roles of the interdisciplinary palliative care team in providing spiritual care is to honor and listen to the suffering of the dying (Sinclair et al., 2006). 


\section{Summary and Discussion}

Modern medicine has allowed many chronically ill people to live longer lives; however, the dying process from a chronic disease can last for years. As the current large group of adults nearing retirement enter old age, the demand for end-of-life care will increase. This literature review examined the history of and today's modern hospice services. Early palliative care leaders, Mount (1976) and Saunders (2000) believed the interdisciplinary palliative care team was essential for the provision of comprehensive care of the patient and family. Hospice programs in the United States are time-limited and funded by federal Medicaid program, which were designed to provide high quality end-of-life care to all.

This literature review has also examined palliative care. Palliative care is the relief of suffering of a patient with life-threatening illness. It may be used for chronic, serious, and life-limiting illness. Palliative care is appropriate for patients of all ages and conditions, and may be short term or long term. Palliative care may be provided wherever the patient lives: at home, in an inpatient hospital unit, skilled nursing home, or in assisted living (Haugen et al., 2010).

Team definition, structure, communication, and collaboration were explained. Palliative care is most often provided by an interdisciplinary team and evidence for the use of interdisciplinary palliative care teams as the standard of palliative care was produced. Bornstein's (2003) two-part model of optimal interdisciplinary collaboration was presented as a conceptual framework for effective interdisciplinary functioning.

Barriers and challenges to interdisciplinary palliative care teams were outlined. Interdisciplinary team dynamics have an impact on the team members. Interdisciplinary 
team, or interprofessional education, is needed in order to teach collaboration. This type of education must be supported at the systems level. It takes work to make interdisciplinary team education effective and this isn't typically recognized in healthcare.

Core components of the interdisciplinary palliative care team were defined. These parts are the patient and family as the unit of care and the four core disciplines of medicine, nursing, chaplaincy, and social work. Each of the four core disciplines were reviewed, and the strengths of each domain, as well as the difficulties of roles and communication within team were discussed. Counselors have the training to be on interdisciplinary palliative care teams, but this discipline is typically not part of the team.

Existential theory as it applies to the dying is the framework for the current study. It is appropriate as many of the dying seek answers to or desire to make meaning from their suffering. Patients and families, and the entire interdisciplinary palliative care team encounter the ultimate existential boundary situation, death, at one's end-of-life. An existential theoretical lens can be helpful to the dying and to those who work with the dying.

Our healthcare system will care for more middle aged adults than ever and they will require capable and knowledgeable workers for their care. As this group reaches the end-of-life, many will receive the services of interdisciplinary palliative care teams who will deliver high quality palliative care. In order to be effective interdisciplinary palliative care team members, these healthcare professionals should be skilled in communication and collaboration as they deliver comprehensive patient and family care. The current study is a qualitative analysis of reflective writings from chaplain interns and social work 
interns who have completed a clinical placement on an inpatient hospital unit interdisciplinary palliative care team.

The next chapter will review the history of the current study and the qualitative methodology used in it. The research design and background of the current study will also be provided. Analysis of de-identified student intern reflective writings and emergent themes used to answer the research questions are also reported. 


\section{CHAPTER III: METHODOLOGY}

\section{Method}

This chapter describes the research design and data collection and analysis procedure about the chaplain interns' and social work interns' written reflections from a select interdisciplinary palliative care team experience. The type of study, history of the current study, and methodology to be used in the current dissertation study will be explained.

As described later in this chapter, all participants in this study are chaplain interns and social work interns who took part in a larger study (i.e., iCOPE study program funded by Robert Wood Johnson Foundation and awarded to the University of Louisville). The chaplain interns' and social work interns' documented experiences are in response to a series of established questions for the iCOPE study (see Appendix A) and serve as the primary data source for this dissertation study. Thus, pre-existing data (e.g., documents and text) from chaplain interns and social work interns about their interdisciplinary palliative care team experience will be used in the current dissertation study to explore the research questions. The research questions to be addressed were as follows.

\section{Research Question 1}

What is the nature of working on an inpatient hospital unit interdisciplinary palliative care team for chaplain interns and social work interns?

The sub-questions associated with this research question were: 
a) What are the documented interdisciplinary team communications and experiences for chaplain interns?

b) What are the documented interdisciplinary team communications and experiences for social work interns?

c) How do the chaplain interns document their communications and experiences with patients and families who are receiving inpatient hospital unit interdisciplinary palliative care?

d) How do the social work interns document their communications and experiences with patients and families who are receiving inpatient hospital unit interdisciplinary palliative care?

\section{Research Question 2}

In what ways does the inpatient hospital unit interdisciplinary palliative care team influence the chaplain interns and social work interns as they work with dying patients and their families?

The sub-questions associated with this research question were:

a) What are the documented thoughts, emotions, and feelings of the chaplain interns as they reflect upon the meaning of their interdisciplinary palliative care experience?

b) What are the documented thoughts, emotions, and feelings of the social work interns as they reflect upon the meaning of their interdisciplinary palliative care experience?

c) How are the described roles and behaviors of the chaplain interns shaped by their interdisciplinary palliative care experience? 
d) How are the described roles and behaviors of the social work interns shaped by their interdisciplinary palliative care experience?

\section{Research Question 3}

How similar are the documented experiences, behaviors, and feelings of chaplain interns to those documented experiences, behaviors, and feelings of social work interns working in inpatient hospital unit interdisciplinary palliative care teams?

\section{Research Design}

This was a qualitative study with its background and methods rooted in phenomenology. The current study explored the documented experiences of chaplain interns and social work interns as they worked with patients in life-threatening and endof-life situations as described in the students' required critical reflective writing assignment for the iCOPE study (see Appendix A). I used a phenomenological approach to study the rich experiences of the chaplain interns and the social work interns as they reflected and documented their interdisciplinary palliative care work.

Phenomenology is the study of lived experiences and its point is to gain deeper knowledge and meaning of ordinary life events (Van Manen, 1990). Moustakas (1994) defined the aim of phenomenology as defining what an experience represents to those who had the experience and are able to describe it. Working with dying patients and their families puts chaplain interns and social work interns in the midst of one of life's great mysteries: death. Van Manen (1990) wrote humans need experiences, reflection, and thought to make life's complex and mysterious nature knowable. In the current study, the interns wrote about the experience of dying from their clinical practice, reflected upon patient interventions and family involvement, and critiqued the treatment plan for a case. 
The originator of phenomenology was a German philosopher, Edmund Husserl, who understood human experiences to be conscious, intentional and "of something" (Valle, 1998, p. 5). Van Manen (1990) explained the belief of intentionality within phenomenology as our intentional act to "become the world" (p. 8). Phenomenology questions what is most essential to know about the world as we live in it (Van Manen, 1990). Husserl's focus was on human consciousness and he sought to answer the question: "what is the essence of this phenomenon?" (Valle, 1998, p. 6).

Alfred Schutz (1970) described consciousness as continuous experience, with one moment moving into the next, with little awareness of what was present and what is past. The recognition of this uninterrupted experience puts one into a state of reflection, which allows for a modification of awareness, which creates a remembrance. For a past experience to be important, it must be reviewed; however, the experience is already past (Schutz, 1970).

Social relationships are integral to daily life and they are shared, experienced, and interpreted by the self and others (Schutz, 1970). Schutz wrote about human encounters and described the "face-to-face" situation (p. 185), which is the connection between two people and their separate streams of consciousness. Each is aware of the other, and the concept of Dasein (i.e., "being-there") is revealed. In this encounter, one human being is intentionally directed to the "being-there" (p. 186) of another conscious human being. Each person interprets their lived experience with the other, in a number of ways; casual, eager, superficial, or personal. The "face-to-face" situation provides a deeper understanding, using intersubjectivity (i.e., an understood assumption that people are 
basically alike) of each person to the other as a result of their shared lived experience (Schutz, 1970).

Schutz was interested in the inner understanding of human experience and the knowledge found from living in the world. Several terms are associated with Schutz's phenomenological approach to lived experience. Epoche or bracketing (p. 317) is a method of inquiry used to put aside one's thoughts and assumptions about the phenomenon under study (Schutz, 1970). Transcendental is defined as the larger world, which includes both the natural world and the social world, in which all humans are placed and take for granted (Schutz, 1970). Two other phenomenological terms that are associated with Schutz are noema and noesis. Noema is what is experienced and noesis is the process of experiencing (Schutz, 1970).

Pertinent to the current study of end-of-life experience, Schutz used a set of time constructs in which we all live our everyday lives. He used the word reach (p. 321) to define one's range of cognitive grasp within the environment (Schutz, 1970). Valle (1990) elaborates on Schutz's concept of reach. Schutz identified the past as the "world within restorable reach," the here and now as the "world within reach," and the future as the "world within attainable reach" (Valle, 1990, p. 9). The dying are especially close to each level of world experience as they may be unusually aware of their past, present, and certain future. The science of phenomenology tries to explain the lived or existential meanings in our human world of everyday existence; in fact, this is research that looks at how we develop more completely into who we are (Van Manen, 1990).

Death may be the ultimate mystery. Van Manen (1990) makes suggestions about the use of phenomenology in the study of human science, which apply to the current 
qualitative study of dying. Human life requires knowledge, reflection, and thoughts in order to reveal its essential unexplained features and this research occurs in the midst of life. Phenomenology seeks to make human science both explicit and have universal meaning. It does not solve problems, but asks meaning questions about lived experience. Good phenomenology presents a clear aspect of the world which is congruent with our experience. Phenomenology is "validated by lived experience and validates lived experience" (Van Manen, 1990, p. 27).

\section{The iCOPE Study}

The current dissertation study was derived from a larger study (i.e., iCOPE study), which began in 2007. The iCOPE program originated from an externally funded grant used for palliative care education from the Robert Wood Johnson Foundation awarded to the University of Louisville, School of Medicine. The program began (Phase I) with third year medical students who were required to complete a palliative medicine clinical rotation. Since July 2008, after the medical students completed their palliative care rotation, researchers from the School of Medicine found the medical students demonstrated significant improvement in palliative medicine skills and possessed increased satisfaction providing end-of-life care when compared to students the previous year who did not complete the rotation (Pfeifer et al., 2012).

Phase I of the research grant was the development of the successful palliative medicine rotation for third year medical students. Following the success of Phase I, the iCOPE team created a larger project, Phase II, to expand the impact of palliative care education. This expanded project included three schools within the University of 
Louisville: School of Medicine, School of Nursing, and Kent School of Social Work (Head et al., 2014).

Phase II of the project also brought in students in chaplaincy programs from three accredited clinical pastoral education centers in Louisville, Kentucky. Thus, Phase II of the study organized third year (a) medical, (b) nursing, (c) social work, and (d) chaplaincy interns into teams who completed five interdisciplinary activities. No counselors were included in the iCOPE study. Once the interdisciplinary educational modules were complete, all interns were expected to demonstrate mastery in palliative care, with communication and teamwork as themes, which supported their learning experiences (Head et al., 2014).

Phase II of the iCOPE study included a written reflective assignment from the students after they completed their interdisciplinary palliative care team clinical rotation at a local inpatient hospital unit (Head et al., 2014; Pfeifer et al., 2012). The interns were required to respond in writing to 10 questions (see Appendix A) regarding their experiences during their clinical rotation. This single reflective writing assignment was required of all students in the iCOPE study and served as the data for the current dissertation study. The students submitted their written reflections, which were approximately three to four pages long, to the iCOPE Program Coordinator, at the end of each semester for the duration of the iCOPE study (i.e., 2012 to 2015).

The current dissertation study used the above-mentioned writing reflections from each chaplain and social work intern who participated in the iCOPE study during 20122015 to explore the research questions. More specifically, Phase II iCOPE study data 
from the 2012-2015 were used due to the data's availability. Importantly, the documents or text data were used because these specifically related to participants' unique clinical experiences working on an interdisciplinary palliative care team, which was the focus of the current study. The next section describes the pilot study that informed the dissertation study.

\section{The Pilot Study}

I initiated a pilot study related to the iCOPE study in fall 2013. More specifically, in preparation for performing qualitative research, I participated in data collection and data analysis coursework within the Palliative Care Fellow Conference of the University of Louisville, School of Medicine during the fall of 2013 and the spring of 2014. As part of the coursework, I conceptualized and carried out a pilot study. That pilot study and experience informed the current dissertation study.

Beginning in September, 2013, I conducted nine weeks of field observation of the Palliative Care Conference. During the weekly two-hour Palliative Care Conference, faculty members from one of the palliative care core areas (i.e., medicine, nursing, social work, chaplaincy) made a presentation, which was followed by one of the following: a)

palliative care case study, b) palliative care journal article review, or c) reflective writing session led by the palliative care fellow or appropriate faculty. I took field notes during all palliative care conference observations.

I also conducted a semi-structured interview with a palliative care physician, who is a faculty member of the University of Louisville, School of Medicine, as well as a practicing palliative care doctor in a local cancer treatment center. After transcribing and analyzing this interview, I became more interested in the issues of training spiritual and 
psychosocial team members (e.g., chaplain interns, social work interns, counselor interns) for palliative care and interdisciplinary palliative care team member collaboration. This interview revealed preparation for the end-of-life can be a long process for the physician as well as the patient. The physician described her connection with patients to be an active listener and provide honest feedback about their remaining time to live.

Following this preliminary work and with Institutional Review Board (IRB) approval, I reviewed the critical reflection writings of chaplain interns and social work interns from 2012 through 2013. As a result of conducting an analysis on the interns' reflections, I wanted to learn more about their experiences as they interacted with and were affected by their dying patients. Findings from the pilot study increased my curiosity in the areas of spirituality and quality of life in the dying, how nonmedical staff (i.e., chaplains, social workers, counselors) become integrated into palliative care teams, and how interdisciplinary palliative care teams function in the hospital.

Analysis of the 2012-2013 chaplain intern reflections included findings in the broad areas of communication and learning as they worked on interdisciplinary palliative care teams. Although no clinical counselors served on the palliative care teams, I believed that a study on chaplain interns and social work interns could be informative to future counselors and counselor education curriculum. This preliminary analysis of chaplain interns' reflective writings served as the impetus for the current dissertation study.

\section{The Current Dissertation Study}

The chaplain interns and social work interns who completed an interdisciplinary palliative care experience within the previously described larger iCOPE study at the 
University of Louisville were the participants from whom the written reflections (i.e., language data) used in the current study are derived. As previously described, the current dissertation study used chaplain interns' and social work interns' reflections collected during 2012-2015 to explore the research questions. These written reflections were responses to 10 questions (see below) regarding their experiences during their clinical rotation in the iCOPE study. This single reflective written assignment was required of all students in the iCOPE study. The interns submit their reflections to the iCOPE Program Coordinator at the end of each semester for the duration of the study. Importantly, the narrative data was used because it specifically related to participants' unique clinical experiences working on an interdisciplinary palliative care team, which was the focus of the current study. The interns respond to the following questions after their clinical palliative care rotation:

\section{¿COPE Critical Reflection Writing and Group Experience}

For this assignment, you are to focus on one patient that you observed during your palliative care clinical experience or from the video (Letting Go, Wit, etc.) that you viewed. Address the areas described below. Your critical reflection paper should not exceed 3-4 pages in length (double-spaced, 12 font, please). Use the template (included) that has been created for this exercise. Include the three sections below.

1. In 1-2 paragraphs, summarize the clinical scenario in language that nonclinical readers could understand.

- Briefly describe the patient and his/her situation, diagnosis and prognosis, brief history of the disease, and how/why the patient entered palliativefocused care.

- Describe the predominant palliative care needs of this patient and his/her family. Think holistically about their medical, psychosocial and spiritual needs.

- Briefly describe how well these needs are currently being met. What team members are involved and what interventions are occurring? How do you judge the progress being made towards the goals for this patient's care? 
2. Now-put on your critical thinking cap. Address the following critical review questions.

- How could the patient's/family care be enhanced?

- Was there an adequate interdisciplinary assessment and care plan?

- Describe the communication among the parties involved (team communication: with each other, the patient and family, and other providers)

- Discuss how this interdisciplinary and patient/family oriented setting supported quality palliative care. If it didn't, how did it fall short?

3. Lastly, but most importantly, reflect personally on what you learned from your involvement with this patient. This should be the longest portion of your paper.

- How did the experience touch you personally? Professionally?

- How did the experience impact your future practice as a healthcare provider?

- Describe observations that had a positive or negative impact on you.

- How has your perception of palliative care changed based on the experience?

Local inpatient hospital units in Louisville hospitals were the sites where the interns completed their palliative care clinical experience. Chaplain interns fulfilled their palliative care clinical experience by shadowing chaplains and social work interns shadowed social workers on interdisciplinary palliative care teams. These placements were on inpatient hospital unit interdisciplinary palliative care teams in Louisville. The hospitals included Louisville Veterans Administration Medical Center, Baptist Health Louisville, University of Louisville Hospital, Norton Hospital, and Kosair Children's Hospital.

The written reflections for the current study were derived from critical semesterending writing assignment from the iCOPE study (Appendix A), were based from real life experiences by the chaplain interns and social work interns, who were iCOPE 
participants, from spring and fall semesters during the 2012 calendar year through the spring semester of 2015 . The reflective writings were routinely collected as required coursework, all interns signed informed consent documents (Appendix B), and research using the reflective writings were approved for study by the University's Institutional Review Board. The interns' reflective writing contributions (i.e., text data) were the focus of this study and were used in analysis and may provide evidence for future placement decisions on interdisciplinary palliative care teams.

I gathered all available chaplain intern and social work intern participant demographic data. I had no connection to the iCOPE office and did not compensate any of the interns for their participation in the reflective writing assignment. There was minimal risk typical of that associated with an educational assignment of reflective writing.

\section{Why Study the Questions?}

Life events, such as trauma and illness, affect patients and their families every day. Hospitals are places where people are sick, and often die, every day (Chambliss, 1996). Chaplains and social workers are core members of hospital interdisciplinary palliative care teams who offer emotional and spiritual support in times of crisis for patients and their families. The chaplain interns and social work interns are supervised by a professional chaplain or professional social worker who is on the on the interdisciplinary palliative care team during their palliative rotation. Each intern observes and learns how to provide support to patients with life-threatening illness or are at the end-of-life and is also exposed to the collaboration within an interdisciplinary palliative care team. 


\section{Why Examine the Current Study's Questions Using Qualitative Methods?}

The central idea of qualitative research was to learn about an issue from the participants' perspective. The study of an interdisciplinary palliative care experience for chaplain interns and social work interns was well-suited for qualitative study because qualitative researchers seek to learn, retell, and interpret the stories of others (Glesne, 2011). Qualitative data describes what it was like to have been present and capture rich descriptions of the experience.

Chaplain interns and social work interns have stories of their encounters with patients in crisis and interdisciplinary palliative care team dynamics. Qualitative methods describe how the interns react and capture portrayals of their experiences. In their review of randomized clinical trials practice-based interventions to improve poor interdisciplinary collaboration, Zwarenstein et al. (2009) suggested more rigorous studies were needed in this area and these should include qualitative methods to add insight into how interventions affect collaboration and if improved collaboration contributes to outcome measures.

Qualitative data often consist of documents that describe what the experience was like (Patton, 2015). The data in the current study were single, required reflective writing assignment documents from each chaplain intern and social work intern who completed an interdisciplinary palliative care rotation within iCOPE. The written reflections followed a critical reflection writing format from iCOPE, which provided open-ended narratives about the interns' palliative care experiences. 
The current study's narrative data (documents and texts) were suitable for qualitative study. These documents were analyzed using a variety of codes, which were arranged into patterns, then grouped into themes, which resulted in a unique combination of findings from the data. Complex reasoning occurs inductively and deductively; researchers build patterns, categories, and themes by organizing them into "increasingly more abstract units of information" (Creswell, 2013, p. 45). The entire study was seen as part of a complex system, which was not reduced to a few rigid variables; it came out of a holistic perspective (Patton, 2015). In qualitative research, the focus of study is the meaning the participants ascribe to the issue, not what the researchers or literature determines it to be.

\section{Role of the Researcher}

In qualitative research, the researcher is a key instrument for research as the critical reflection writing documents are examined (Creswell, 2013). As the sole researcher, my role was to assemble and analyze the data. The data from the current study was read in order to understand its context and then to organize it into codes, patterns, and themes. I strived to employ empathetic neutrality during the current study. Empathetic neutrality is defined as the middle ground between over involvement, which may affect judgment, and overly distant, which reduces awareness and insight (Patton, 2015). I did not physically enter the environment, which in this case, was the inpatient hospital units, nor did I conduct interviews to collect data. My personal connection to the data was a distant one, so I was mindful that my awareness and insight could be lessened. 


\section{Bias}

The credible qualitative researcher is required to think about, handle, and report sources of bias and error (Patton, 2015). Researcher bias occurs from a lack of awareness of the ways one's personal views may affect how data are interpreted (Johnson, 1997) and the objective researcher makes assumptions and biases obvious to self and to others (Morrow, 2005). I recognized my positive personal bias toward the subject of palliative care as I undertake this study.

For example, palliative care is an area of human experience in which I have great interest. I have worked in the bereavement field and have grieved over lost family members, friends, and pets. My mother received palliative care during the last week of her life. I have never worked on a palliative care interdisciplinary team. In my role as a bereavement counselor, I worked with survivors who had typically received interdisciplinary palliative care team support. I endorse the integration of disciplines that form the interdisciplinary team as the foundation of comprehensive palliative care and the holistic support given to patients and their families at the end-of-life.

Qualitative researchers also recognize their interpretations of events and experiences that are under study are affected by researcher bias. An important approach to minimize bias is to employ reflexivity. Johnson (1997) defines reflexivity as "active critical self-reflection about potential biases and predispositions" (p. 284) and by using reflexivity, researchers become more self-aware as they monitor and try to control their biases.

A helpful strategy for self-reflection, or reflexivity, is to write analytic memos. Saldana (2009) wrote that analytic memos can become prompts from written reflections 
for the purpose of critical thinking about why and what you are doing. Analytic memo writing is a way for the researcher to record thoughts during data analysis, creative thinking or other thoughts, ranging from insights during coding to frustrations with analysis (Saldana, 2009).

I wrote analytic memos and used them for contemplation during data analysis. These self-reflective memos are available for examination and may be used during any portion of the research process as a record of experiences that may inform data analysis or findings. Writing analytic memos can capture emergent ideas that are generated during all phases of data analysis.

Reflexivity also includes the researcher's self-awareness of how knowledge is constructed from the research process and how this self-awareness affects every stage of the research, which promotes the rigor of a study (Guillemin \& Gillam, 2004). In this study, I attempted to remain critically aware of the factors that influence how I approached, interpreted the data, and reported my findings. Etherington (2007) considered reflexivity to also include the power the researcher possesses in representing and interpreting data.

Qualitative researchers develop a complex, holistic, and broad picture of the issue under study. The findings from a qualitative study are not generalizable to other groups; however, they may be useful to identify larger themes, which could be applied in similar settings (Patton, 2015). In the current study of chaplain interns' and social work interns' experiences with their dying patients and interdisciplinary palliative care team placement, analyses of their written reflections (i.e., text data) brought awareness to the meaning of 
their encounters. A study of this type may bring forth different information not found in typical outcome-based evaluations.

\section{Decisions Guiding Methods}

A strong qualitative study uses rigorous methods at all phases of planning and research. Creswell (2013) suggested characteristics of a rigorous qualitative study are demonstrated when the researcher uses a recognized approach to the study; in this case, methods of phenomenological design was the framework for the current study. A qualitative study explores a central phenomenon and develops a detailed exploration and understanding of the phenomenon (Creswell, 2012).

In the current study, the core concept was the interdisciplinary palliative care team experience for chaplain interns and social work interns who worked with patients with life-threatening illness or were at the end-of-life. Another way to enhance the rigor of a study is to use multiple layers of abstraction; which is to analyze the data using smaller, defining codes which are then grouped into categories, patterns or themes, then culminate in abstract and general findings. I used all data, especially data that were unusual or not easily fit into patterns. Often these data give a study unexpected information and reflect the complexity of real life (Saldana, 2009).

\section{Sampling}

Another characteristic of qualitative design that fit this study was purposeful sampling, which is using the group who experiences the phenomenon to be under study. The group of chaplain interns and social work interns are an "information rich" (Patton, 2015, p. 53) sample who can offer useful insights about the phenomenon. The use of 
purposeful sampling allows the researcher to study the phenomenon in-depth (Patton, 2015) from two groups, which are homogeneous by occupation.

The participants from whom the data were derived were iCOPE participants (i.e., chaplain interns and social work interns). The focus of this study was the language data (documents and texts) derived from select iCOPE participants' required reflective writings. The narrative data for this study were personal reflections from the interns' lived experience as members of hospital interdisciplinary palliative care teams, experienced in the natural setting of the hospital.

The qualitative method does not produce findings that are generalizable as do the random sampling techniques used in quantitative methods; however, the insights gained from purposeful sampling may provide a great amount of information, which may be useful for educational decisions regarding the chaplain interns and social work interns. The current study sample participants were derived from a larger, ongoing study (e.g., iCOPE study).

\section{Confidentiality}

I maintained confidentiality and anonymity as I do not know the names of any of the interns because their reflective writings were de-identified before I received them. Within the terms of research, confidentiality also means presenting findings in ways that prevent the identification of the interns (Wiles, Crow, Heath \& Charles, 2008), which means being aware of providing too much contextual information that could lead to identification. As a qualitative researcher, it is my responsibility to assure the ethical standards of my study using ethical sensitivity and judgment (Colnerud, 2013). 


\section{How I Gained Entry}

I received the chaplain intern and social work intern reflective writing assignments through the co-primary investigator of the iCOPE study, who is an Associate Professor of Medicine at the University of Louisville. She is also the Director of the Interdisciplinary Program for Palliative Care and Chronic Illness within the University of Louisville, School of Medicine. I requested reflective writing data from the department of Interdisciplinary Curriculum for Oncology Palliative Care Education, through the coprimary investigator for my qualitative pilot study, which began in 2012. I received IRB approval (Reference numbers: $370683 \& 429501$ ) to use the data for my current dissertation study. I received permission to use the de-identified by name reflective writings of the chaplain interns and social work interns for fall and spring semesters from 2012 through the spring semester of 2015.

\section{Data}

Data collection is more than what the actual data are and how one will obtain it. Creswell (2013) explained data collection is a multifaceted process. Data collection is obtaining permissions, getting a representative sampling of data, developing ways of recording data, storing the data, anticipating possible ethical problems, and having safeguards in place to handle accidental and unplanned violations. The reflective writings were anonymous, which protected the interns, members of the interdisciplinary palliative care teams, and their patients. I was aware of using contextual identifiers (e.g., names of hospitals and locations) as I discussed the content of the writings that could lead to identification of interns or their patients (Colnerud, 2013). 
In the current study, the data were the chaplain intern and the social work intern reflective writings, which were written using the required iCOPE Reflective Writing Assignment format. The written reflections were required during the semester in which the interns participate on their interdisciplinary palliative care clinical experience. The reflections (i.e., text data) were submitted to the iCOPE Program Coordinator once per semester within the academic timeframe of the disciplines. These reflections were typically three to four pages long.

Deidentified data (i.e., chaplain intern and social work intern written reflections) were downloaded on a flash drive by Amy Martin, R. N., Clinical Research Nurse, Interdisciplinary Program for Palliative Care and Chronic Illness. Demographic data (e.g., age and gender), as well as the discipline, timeframe, and setting connected to each chaplain intern and social work intern reflective writing were also provided. All data (i.e., narrative reflective writings) were kept on a flash drive, which was considered to be electronic master copies and were backed up by an external hard drive.

Hard copies were made by printing all reflective writings. Patton (2015) recommended protecting data by making backup copies of data and to secure these copies in a safe place. An additional set of copies were made of all reflective writings and these copies were the master hard copies, which were placed in a locked cabinet. Another hard copy was used for writing on (e.g., making codes) for data analysis.

I documented the analysis process daily in order to provide the foundation of rigor required in qualitative analysis (Patton, 2015). Writing analytic memos can become prompts for critical thinking and they can capture emergent ideas that are generated during all phases of data analysis (Saldana, 2009). I also used reflexivity, which is critical 
reflection on bias and predisposed ideas, in order to increase my awareness of preconceived thoughts (Johnson, 1997).

\section{Inclusion and Exclusion Criteria}

In order to be included in the current study, the documents had to be written by an intern who had a real interdisciplinary palliative care experience. Participant documents who used a virtual patient or did not have an interdisciplinary experience were excluded. All written responses put forward by the interns were used, unless the responses to the 10

questions (see Appendix A) were less than two pages, interns self-identified as other than chaplain intern or social work intern, or were not understandable or interpretable (e.g., not written in English).

\section{Data Analysis}

\section{First Cycle Coding}

All coding analysis described was conducted by hand. First Cycle Coding methods were as follows:

Step 1: Attribute coding of all reflections. The first step in data analysis of the reflective writings was to assign Attribute Codes, which provided essential information about participants and qualitative data management (Saldana, 2009). The narrative data (i. e., reflective writings of chaplain interns and social work interns) were numbered and attribute coded using the following codes: discipline, age, gender, and setting.

Step 2: Initial reading of the reflections. All chaplain intern $(\mathrm{N}=24)$ and social work intern $(\mathrm{N}=23)$ reflections were read through once, in order to gain a sense of the environment where they occurred. The written reflections were variable in their responses to the iCOPE Reflective Writing assignment questions. During the first reading of the 
reflections, I found some of the reflective writings gave detailed and specific information to the questions from the iCOPE Reflective Writing Assignment, while other reflective writings were more general in their responses.

Step 3: First cycle coding. During the second reading of each reflection I developed a preliminary list of codes and short phrases. Initial Codes, Emotion Codes, In Vivo Codes, and Values Codes were used during First Cycle Coding. Saldana (2009) explained the rationale for each type of code. Initial Codes break down data into parts and are appropriate for all qualitative studies, especially for beginning researchers using data in document form. In Vivo Codes are appropriate for nearly all qualitative studies, but specifically useful when the researcher wants to highlight and honor the participant's voice. Emotion Codes label emotions experienced by the interns as they participated with palliative care patients and Values Codes are used to code values, beliefs, and attitudes that represent the intern worldview.

Step 4: Organization and coding of responses within the reflections. After First Cycle Coding was complete, the codes and short phrases were then typed for each chaplain intern and social work intern. The codes and short phrases were grouped together by code type (e.g., Initial, Values, Emotion, or In Vivo). The same types of codes were used for all reflection writing coding and the codes were separated by discipline (i.e., chaplain interns and social work interns).

\section{Second Cycle Coding}

Second Cycle coding methods are advanced ways of reanalyzing and reorganizing the data after First Cycle coding (Saldana, 2009). Miles, Huberman, and Saldana (2014) used the term data condensation, rather than reduction, which is analysis that sharpens, 
focuses, and organizes data into developing themes from which conclusions may be drawn.

Step 5: Themes from second cycle codes. Using the codes and short phrases from each chaplain intern and social work intern reflective writing, created in Step 4, I organized all codes and phrases into broad themes. The codes and phrases found in the reflection of each participant (i.e., chaplain intern or social work intern) were read and placed in a larger category. All codes and short phrases were collected from each chaplain intern and social work intern and placed in themes or patterns which were discipline-specific.

Saldana (2009) defined Focused coding as a Second Cycle coding method, which searches for the most frequent Initial Codes from First Cycle coding. Using this approach, the most prominent categories and themes were then developed from the data. Seven themes and six subthemes emerged from the data.

The themes contained within the data are: Learning Lessons, Disappointment, Being There, Dealing with Family Matters, Making Hard Decisions, Intern as Existential Comforter, and Intern as Psychosocial Resourcer. These themes explored how both groups of interns learned to work on an interdisciplinary care team and how the team functions. Knowledge was also acquired for both groups as they worked with patients and their families at the end-of-life. The chaplain interns and the social work interns also gained personal insights, discipline-specific awareness, and new information about palliative care patients. 
Step 6: Completion of data analysis. Themes and subthemes from data analysis of the written and were reflections used to answer the research questions and subquestions.

\section{Reliability and Verification in Data Analysis}

\section{Analytic Memos}

I wrote my thoughts, worries, and reflections on data analysis every day I worked with the data. I reviewed my analysis and reorganized my data several times during Second Cycle Coding. These steps added more themes and subthemes, which further enhanced the findings. Miles et al. (2014) suggested analytic memos be separate from data and kept as a separate document. My analytic memo writings were kept in a notebook, unconnected to my data.

\section{Reliability}

The current study required analysis that fit its specific situation, which in this case was a single researcher working with chaplaincy intern and social work intern reflective writing assignments. It is the responsibility of the qualitative researcher to fairly represent the data because there are no absolute rules that apply to ensure reliability and validity (Patton, 2015). Researchers who conduct reliable studies are obligated to be explicit when describing their process, which means maintaining a detailed accounting, or audit trail of research activities, emergent themes, categories, and analytic memos that may be reviewed by peers or advisors (Morrow, 2005). I kept a daily account of research activities, analytic memos, and critical thoughts and questions during data analysis, to add to the reliability of my study. 


\section{Verification}

Johnson (1997) suggested searching for negative cases during analysis and using low inference descriptors in the findings as ways to promote accuracy and credibility. Negative case sampling is reporting of evidence that does not neatly fit into codes or themes in analysis (Glesne, 2011). Low inference descriptors are direct quotations that help the reader experience the interns' actual language and meaning of the event (Johnson, 1997).

In order to enhance the validity or trustworthiness of my research, I searched for negative cases in analysis. I found three cases (i.e., two chaplain intern cases; $\mathrm{CH} 4, \mathrm{CH} 7$ and one social work intern case; SW7) where the interns had no experience with palliative care as they cared for dying patients. The patients from these written reflections received adequate medical care from other hospital departments (e.g., oncology or intensive care units), but no palliative care in their last days. In these cases, the interns and the unit chaplains or oncology social workers gave support to the patients. The interns wrote about these interdisciplinary experiences in their reflected writings.

I made use of low inference descriptors in the answers to my research questions, which improves the validity of my research. I applied the interns' own words to describe situations, thoughts, and feelings they experienced as participants on the interdisciplinary palliative care team. Using direct quotes honored the voice of the intern as they reflected upon their encounters.

\section{Ethical Considerations of Human Subjects}

Research using critical reflective writings from each intern who completed an interdisciplinary palliative care rotation were approved by the University of Louisville's 
Human Subject Protection Program (HSPP) and IRB (Reference numbers 370683 \& 429501). Each intern signed an Informed Consent form (Appendix B) from the iCOPE office, which explained the purpose, procedures, potential risks, and benefits of participation in the iCOPE study. The consent form stated confidentiality or total privacy cannot be guaranteed; however, the interns' privacy is protected to the extent permitted by law and published results will not use any interns' names or personal identifying information (Pfeifer et al., 2012). Before data analysis began on the current study, University of Louisville IRB approval was received. As previously mentioned, the current study used de-identified by name intern narrative reflections from the iCOPE study as data in the current dissertation study.

This chapter stated the research questions and rationale for using qualitative methods for the project. Analysis of de-identified by name intern reflective writings were used to answer the research questions. To be considered trustworthy and credible, a qualitative study must use strategies to enhance both reliability and validity in data collection and data analysis. This chapter reviewed the history of the current study and the qualitative methodology used in it. The next chapter reports the findings. 


\section{CHAPTER IV: FINDINGS}

This chapter presents information about the study sample and describes the findings from the chaplain intern and social work intern participants' interdisciplinary palliative care team experiences derived from the data reported during their end of the semester iCOPE reflective writings. Six themes were developed from the chaplain intern reflective writings. Similarly, six themes were also developed from the social work intern reflective writings. These themes informed the answers to the dissertation study research questions.

Understanding the meaning of the lived experiences of chaplain interns and social work interns from an interdisciplinary palliative care team rotation was determined from the findings. Clarifying the extent to which the reported roles, responsibilities, and communications were similar or different between the intern groups was also explored. The research questions that were examined are described in the following paragraphs.

Research Question 1 was What is the nature of working on an inpatient hospital unit interdisciplinary palliative care team for chaplain interns and social work interns?

The sub-questions associated with this research question were:

a) What are the documented interdisciplinary team communications and experiences for chaplain interns?

b) What are the documented interdisciplinary team communications and experiences for social work interns? 
c) How do the chaplain interns document their communications and experiences with patients and families who are receiving inpatient hospital unit interdisciplinary palliative care?

d) How do the social work interns document their communications and experiences with patients and families who are receiving inpatient hospital unit interdisciplinary palliative care?

Research Question 2 was In what ways does the inpatient hospital unit interdisciplinary palliative care team influence the chaplain interns and social work interns as they work with dying patients and their families?

The sub-questions associated with this research question were:

a) What are the documented thoughts, emotions, and feelings of the chaplain interns as they reflect upon the meaning of their interdisciplinary palliative care experience?

b) What are the documented thoughts, emotions, and feelings of the social work interns as they reflect upon the meaning of their interdisciplinary palliative care experience?

c) How are the described roles and behaviors of the chaplain interns shaped by their interdisciplinary palliative care experience?

d) How are the described roles and behaviors of the social work interns shaped by their interdisciplinary palliative care experience?

Research Question 3 was How similar are the documented experiences, behaviors, and feelings of chaplain interns to those documented experiences, behaviors, and feelings 
of social work interns working in inpatient hospital unit interdisciplinary palliative care teams?

\section{Theoretical Framework}

A qualitative study explores a central phenomenon and develops a detailed exploration and understanding of it (Creswell, 2012). This was a qualitative research study that used reflective writing documents to examine the meaning of being a chaplain intern or a social work intern on an interdisciplinary palliative care team. The interns learned about the experience of dying from their clinical practice, gained reflection from discussing patient interventions and family involvement with their interdisciplinary discussion groups, and wrote critical reflections about a case, including the thinking that accompanies these activities.

This study was rooted in a phenomenological approach. Phenomenology is the study of lived experiences in order to acquire meaningful knowledge of ordinary life events (Van Manen, 1990). Those who lived the experience and can describe it represent the purpose of phenomenology (Moustakas, 1994). The chaplain interns and the social work interns lived the experience of working with dying patients and their families that put them in the midst of one of life's great mysteries: death.

Death, which will most certainly affect everyone, may be the ultimate mystery. Questions about ordinary events, such as death, may cause reflection on the experience to determine its meaning (Van Manen, 2014). Phenomenology seeks to make human science both explicit and have universal meaning. It does not solve problems, but seeks to clarify the meaning of the lived experience of a phenomenon for a group of people (Patton, 2015). 
Humans need experiences, thoughts, and reflection to make life's mysterious and complex nature knowable (Van Manen, 1990). A phenomenological framework explores lived experience. This method of inquiry illustrates the experiences of chaplain interns and social work interns as they work with patients at the end-of-life. It also informs the researcher about experiences both groups of interns have as they participate as members of interdisciplinary palliative care teams.

\section{Characteristics of the Sample}

The current study was composed of the iCOPE Reflective Writing Assignment data (i.e., reflections) from chaplain interns and social work interns from the following semesters: Spring 2012 through Spring 2015. For specific details related to the sampling

frame see Appendix D and Appendix E. All patients with whom the chaplain interns and social work interns worked were adults. There were no pediatric patients seen by this sample of chaplain interns and social work interns. The total number of pages used for analyses were 157.5.

\section{Description of Chaplain Intern Sample}

The sample size of the chaplain interns was $\mathrm{N}=24$, with 12 males and 12 females. The ages of the chaplain intern sample ranged from 25 years to 60 years, with a mean age of 41 years. There were four hospital settings where the chaplains completed their palliative care clinical rotation including: Norton Hospital $(\mathrm{n}=12)$, Audubon Hospital ( $\mathrm{n}=1$ ), Baptist Hospital Louisville $(\mathrm{n}=5)$, and University of Louisville Hospital $(n=4)$. Two chaplain interns did not identify their setting and two others did not provide their ages for demographic information (see Appendix D for further detail). 


\section{Description of Social Work Intern Sample}

The sample size of the social work interns was $\mathrm{N}=23$, with two males and 21 females. The ages of the social work intern sample ranged from 23 years to 60 years, with a mean age of 29 years. Two social work interns did not provide their ages. The social work interns were at hospitals, Hosparus (i.e., the local hospice agency) and other cancer care related settings. The hospitals represented by the social work interns were: Norton Hospital $(n=4)$, Baptist Hospital Louisville $(n=6)$, University of Louisville Hospital (n =4), and Louisville Veterans Administration Medical Center $(\mathrm{n}=2)$. The interns were also in palliative settings, such as Hosparus $(n=5)$, the office of Consultants in Blood Disorders and Cancer $(n=1)$, and the Louisville chapter of Gilda's Club $(n=1)$.

\section{iCOPE Reflective Writings}

The data for the current study were derived from the written reflective assignments for a larger, ongoing study described previously (iCOPE study) comprised of chaplain interns and the social work interns after they completed their interdisciplinary palliative care team clinical rotation. More specifically, the interns were required to respond in writing to 10 questions (see Appendix A) regarding their experiences during their clinical rotation. This one-time reflective writing assignment was required of all students in the iCOPE study and served as the data for the current dissertation study. The students submitted their written reflections, which were approximately three to four pages long, to the iCOPE Program Coordinator at the end of each semester.

The current dissertation study used the above-mentioned writing reflections from each chaplain and social work intern who participated in the iCOPE study during 20122015 to explore the research questions. Importantly, the documents or text data were used 
because it specifically relates to participants' unique clinical experiences working on an interdisciplinary palliative care team. The interns' distinctive interdisciplinary team placements provided the focus of the current study.

\section{Overall Findings}

\section{Chaplain Intern Themes}

The chaplain interns in the current study completed the iCOPE Reflective Writing Assignment where they responded to questions about their interdisciplinary palliative care experience. In the iCOPE Reflective Writing Assignment (Appendix A) they were asked to write about a case in which they participated. The chaplain interns wrote about the patient's health history and current diagnosis and prognosis. They were also asked to determine if they believed the comprehensive palliative care needs of the patient and the family were being met. Critical thinking about the care provided for patient and family, adequacy of interdisciplinary assessment, plan of care, team communication, and team communication for the patient and family is addressed. Lastly, the interns wrote about what they learned from their involvement with their patient.

Six themes were found in the chaplain interns' reflective writings. Each theme was reviewed and studied throughout data analysis to inform understanding of the shared experiences of the chaplain interns. The six emergent themes were: Learning Lessons,

Disappointment, Being There, Dealing with Family Matters, Making Hard Decisions, and Intern as Existential Comforter. Three themes (i.e., Learning Lessons, Being There, and Dealing with Family Matters) were further categorized, each using two subthemes. Table 1 describes the themes that were used to answer the study research questions about the chaplain interns. 
Table 1.

Chaplain Intern Thematic Outline

\begin{tabular}{|c|c|}
\hline Themes & Meanings of Themes and Subthemes \\
\hline $\begin{array}{l}\text { Theme 1: } \\
\text { Learning Lessons }\end{array}$ & $\begin{array}{l}\text { This theme encompasses the knowledge the interns realized during } \\
\text { their clinical placements. Education was acquired in two subtheme } \\
\text { areas: } \\
\text { - Learning Lessons: Being a chaplain } \\
\text { - Learning Lessons: From the team } \\
\text { Examples of the subthemes are learning what is important to } \\
\text { teamwork and discovering what it is like to work as a chaplain } \\
\text { intern from the placement. Chaplain intern feelings about their } \\
\text { placements are also in this theme. }\end{array}$ \\
\hline $\begin{array}{l}\text { Theme 2: } \\
\text { Disappointment }\end{array}$ & $\begin{array}{l}\text { Communication breakdowns produce the theme of } \\
\text { Disappointment. These communication failures may occur between } \\
\text { clinicians, team members, medical services, and/or patient and } \\
\text { family at the end-of-life. }\end{array}$ \\
\hline $\begin{array}{l}\text { Theme 3: } \\
\text { Being There }\end{array}$ & $\begin{array}{l}\text { This is a theme that includes the wide range of experiences the } \\
\text { interns gained at their interdisciplinary clinical placements. These } \\
\text { events were further divided into two subthemes: } \\
\text { - Being There: With the team } \\
\text { - Being There: For the patient and family }\end{array}$ \\
\hline $\begin{array}{l}\text { Theme 4: } \\
\text { Dealing with Family } \\
\text { Matters }\end{array}$ & $\begin{array}{l}\text { This theme contains two subthemes: } \\
\text { - Dealing with Family Matters: Complicating factors } \\
\text { - Dealing with Family Matters: Simplifying factors } \\
\text { Examples of each subtheme are family disagreement over } \\
\text { treatment or patient and family harmony about desire for end-of- } \\
\text { life care. }\end{array}$ \\
\hline $\begin{array}{l}\text { Theme 5: } \\
\text { Making Hard Decisions }\end{array}$ & $\begin{array}{l}\text { Families must often make difficult and final choices for the patient } \\
\text { when death is imminent. This theme contains how these decisions } \\
\text { were made and how the chaplain interns were part of this process. }\end{array}$ \\
\hline $\begin{array}{l}\text { Theme 6: } \\
\text { Intern as Existential } \\
\text { Comforter }\end{array}$ & $\begin{array}{l}\text { This theme contains the unique contributions chaplain interns make } \\
\text { on the interdisciplinary care team, their roles, and their } \\
\text { interventions for the patient and family. }\end{array}$ \\
\hline
\end{tabular}

\section{Chaplain Intern Thematic Categories}

The themes overlapped and easily aligned with each other as the chaplain interns

all answered the same probes. Each chaplain intern answered the reflective writing

assignment; however, not every chaplain intern's narrative demonstrated all presented

themes. Three themes, Being There, Learning Lessons, and Intern as Existential 
Comforter had roughly an equivalent number of codes to comprise the theme. The final three themes, Making Hard Decisions, Disappointment, and Dealing with Family Matters had fewer codes to construct them and thus, fewer illustrative quotes. Not every chaplain intern experienced a family who chose to remove their loved one from a ventilator, witnessed a communication failure, or worked with a positive or negative family environment.

\section{Social Work Intern Themes}

Completing a reflective writing assignment about their interdisciplinary palliative care experience was also a requirement of the social work interns in the current study. In the iCOPE Reflective Writing Assignment (Appendix A), they were asked to write about a case in which they participated. The assignment was expected to contain the background information of the patient, including health history, current diagnosis, prognosis, comprehensive palliative care needs of the patient and the family. The social work intern determined whether the patient's palliative care requirements were being met. As such, the assignment asked for critical thinking focused on the following questions: Is care provided for patient and family? Meaning is there adequate interdisciplinary assessment, plan of care, team communication, and team communication with the patient and family?

Within the assignment, the social work interns wrote about what they learned from their clinical placements. Six themes were found in their reflective writings. Each theme emerged from data analysis and was reviewed to offer an understanding of the shared experiences of the social work interns. The six emergent themes were: Learning Lessons, Disappointment, Being There, Dealing with Family Matters, Making Hard 
Decisions, and Intern as Psychosocial Resourcer. Table 2 describes the themes that were used to answer the study research questions about the social work interns.

Table 2.

Social Work Intern Thematic Outline

\begin{tabular}{|c|c|}
\hline Themes & Meanings of Themes and Subthemes \\
\hline $\begin{array}{l}\text { Theme 1: } \\
\text { Learning Lessons }\end{array}$ & $\begin{array}{l}\text { This theme encompasses the knowledge the interns realized } \\
\text { during their clinical placements. Education was acquired in } \\
\text { two subtheme areas: } \\
\text { - Learning Lessons: From the team } \\
\text { - Learning Lessons: Being a social worker } \\
\text { Examples of the subthemes are learning what is important } \\
\text { to teamwork and discovering what it is like to work as a } \\
\text { social work intern from the placement. Social work intern } \\
\text { feelings about their placements are also in this theme. }\end{array}$ \\
\hline $\begin{array}{l}\text { Theme 2: } \\
\text { Disappointment }\end{array}$ & $\begin{array}{l}\text { Communication breakdowns between clinicians, team } \\
\text { members, services, and/or patient and family at the end-of- } \\
\text { life comprise this theme. }\end{array}$ \\
\hline $\begin{array}{l}\text { Them } \\
\text { Being }\end{array}$ & $\begin{array}{l}\text { This is a theme that includes the wide range of experiences } \\
\text { the interns gained at their interdisciplinary clinical } \\
\text { placements. These events were further divided into two } \\
\text { subthemes: } \\
\text { - Being There: With the team }\end{array}$ \\
\hline $\begin{array}{l}\text { Theme 4: } \\
\text { Dealing with Family } \\
\text { Matters }\end{array}$ & $\begin{array}{l}\text { This theme contains two subthemes: } \\
\text { - Dealing with Family Matters: Complicating factors } \\
\text { - Dealing with Family Matters: Simplifying factors } \\
\text { Examples of each subtheme are family disagreement over } \\
\text { treatment or patient and family harmony about desire for } \\
\text { end-of-life care. }\end{array}$ \\
\hline $\begin{array}{l}\text { Theme 5: } \\
\text { Making Hard Decisions }\end{array}$ & $\begin{array}{l}\text { Families must often make difficult and final choices for the } \\
\text { patient when death is imminent. This theme contains how } \\
\text { these decisions were made and how the team supported the } \\
\text { patient and family }\end{array}$ \\
\hline $\begin{array}{l}\text { Theme 6: } \\
\text { Intern as Psychosocial } \\
\text { Resourcer }\end{array}$ & $\begin{array}{l}\text { Contributions and roles that are expected of social workers } \\
\text { and are often associated with the social work discipline } \\
\text { comprise this theme. }\end{array}$ \\
\hline
\end{tabular}

\section{Social Work Intern Thematic Categories}

The emergent themes from the social work intern reflective writing assignment corresponded and related to each other. The social work interns gave answers to the 
probes within the assignment; but not all social work intern reflections can be interpreted to include all themes. As with the chaplain intern written reflections, three social work intern themes, Learning Lessons, Being There, and Intern as Psychosocial Resourcer had nearly equivalent numbers of codes which make up the theme. The other three themes, Making Hard Decisions, Disappointment, and Dealing with Family Matters had fewer codes within each category, therefore, fewer expressive references. These differences can be attributed to dissimilar interdisciplinary care team experiences within the social work intern group.

\section{Findings: Emergent Themes}

Many experiences comprise the nature of working on an interdisciplinary palliative care team. Working with team members is one way for the chaplain interns and social work interns to achieve practical knowledge of interdisciplinary team dynamics. How to practice as a chaplain or social worker with patients and their families is another area where the interns gain useful learning. The interdisciplinary team incorporates the assessments and clinical knowledge of all team members as well as the patient's goals to achieve a comprehensive plan of care, which is used to guide interventions.

The interns shadowed a chaplain or a social worker on the interdisciplinary team who performed disciplinary-specific work as well as functioned as a member of the team. The chaplain interns often assessed patients for spiritual distress, spiritual support, and religious preferences, whereas the social work interns saw how to conduct a family session or locate placements for patients. All themes and subthemes were gleaned from data analysis of written reflections from the interns. The following themes were representative of chaplain intern and social work experience. 
All chaplain interns worked in hospitals (see Appendix D). The pace in a hospital can be hectic, especially during an emergency. Some of the chaplain interns described emergencies, which were fast paced, and others described palliative care, with a much slower and deliberate rate.

Likewise, the social work interns were most often located in hospitals; though several were assigned to facilities that provided interdisciplinary care for cancer patients or made hospice home visits. The social work interns also saw how to aid families as they struggled with financial, emotional, and social issues, while they coped with the death of a loved one. Social support, in the forms of housing assistance, aid for heating a home, or providing death and dying activities for families and their young children, provided by social workers can benefit family members.

Comfort and ease are often achieved during end-of-life care, and both groups of interns learned ways to provide relief during their clinical placements. Pastoral care is provided in an environment where there is little support from family or situations that do not run smoothly. Social workers also learned how to care for the dying and their families at the end-of-life. The nature of working on the interdisciplinary team for chaplain interns and social work interns is learning how to care for the dying and how to provide support to those who need it, especially when end-of-life situations don't go as planned.

The next sections outline the themes which emerged from data analysis for both groups of interns. The interns were influenced by the interdisciplinary palliative care team as they interacted with the patient and family. Also, similarities between the chaplain interns and social work interns exist. 


\section{Theme 1: Learning Lessons}

This section refers to acquisition of knowledge the interns gained while at their interdisciplinary team clinical placement. This theme was further divided into subthemes: Learning Lessons: From the Team, Learning Lessons: Being a Chaplain, and Learning Lessons: Being a Social Worker. The purpose of the interdisciplinary care team clinical placement for the chaplain interns and social work interns was to provide an actual interdisciplinary care team experience. The first subtheme was associated with knowledge acquired by the intern as a result of being a team member of the team and the examples which follow typify the intern experience. The subsequent subthemes explored the knowledge acquired by the chaplain interns and the social work interns as they performed their interdisciplinary duties and spent time with the patient and their family.

Subtheme 1a: Learning lessons: From the team. Members of the interdisciplinary team communicate with each other so all patient care needs are covered. A chaplain intern wrote about her experience with her patient as she learned interdisciplinary care rounding with the team $(\mathrm{CH} 12)$. The patient required pain management and emotional support to deal with her young adult children who are unable to be in the same room together.

Family issues required all members of the team to help the patient cope. In this case, the patient's blood pressure had risen, presumably in anticipation of conflict from her children, and the chaplain intern discussed options for handling their presence with her. The patient asked for a prayer, which was offered by the chaplain intern. The chaplain intern reflected, "While this patient's primary concern was reducing her pain, everyone on the team provided a different aspect of care aimed at the same thing- 
improving quality of life" $(\mathrm{CH} 12)$. The chaplain intern saw the team work together to provide comprehensive care for the patient to reduce her level of pain and also give emotional support for her difficult family context.

Social work interns also learned the dynamics of interdisciplinary palliative care teamwork by being on the team. Interdisciplinary care comprehensively manages the patient's needs and goals in accordance with the plan of care. During clinical placement, one intern learned how helpful interdisciplinary palliative care can be for the patient, especially when the patient received palliative care very late in his disease. He stated, It is not unusual for palliative care teams to be considered until it is too late. In many cases palliative care will transition into hospice care referrals due to primary care doctors not wanting to use palliative services until the last possible moment. In the case of this patient, who had been receiving treatment for his cancer for over a year, the palliative team would have been a beneficial service for him and his family (SW20, para. 4).

A chaplain intern wrote about his experience where comprehensive patient care from an interdisciplinary team did not occur because the patient's unit does not provide one. In his reflection, the chaplain intern described a patient who received medical and spiritual care, but not palliative care. The hospital admitted the chaplain intern's patient into the intensive care unit, where his condition worsened, and he was placed on a ventilator $(\mathrm{CH} 7)$.

In this chaplain intern's opinion, the patient did not receive sufficient pain or symptom management. His symptoms included shortness of breath, headaches, and lack of sleep. Because there was no interdisciplinary palliative care team for him, 
comprehensive goals of care were never discussed with him when he was able to convey his thoughts before his condition caused him to be placed on a ventilator ( $\mathrm{CH} 7)$.

The chaplain intern described uncoordinated care that compromised the patient's quality of life and autonomy when decisions needed to be made, which resulted in suboptimal care for him. According to the chaplain intern's writings, the ethically responsible solution for quality care are in-patient palliative care services provided by a committed interdisciplinary care team. After he saw his patient receive inadequate and disjointed care, he stated clear support for interdisciplinary care: "Through its patientcentered, interdisciplinary, and holistic approach, hospitals with palliative care services fulfill their ethical obligation to provide best quality beneficial care to all patients" $(\mathrm{CH} 7)$.

As the following patient neared active dying, the interdisciplinary team shifted the focus of treatment and provided emotional support to him and his family. The team arranged for a hospice volunteer to perform a ceremony which recognized the patient's military service in the Army. The social work intern saw the influence of this team decision to thank the patient for serving his country (SW1).

The social work intern described the ceremony for the patient and what she gained from it:

I think this is the first time I began to have a deeper appreciation for the services of the U. S. armed forces. I never had a deep connection to the U.S. military because it is a foreign military to me and having grown up in a neutral country with a militia army I never understood the concept of appreciating military service. During the ceremony, I realized the patient, who was stationed in 
Germany, was cleaning up my continent and I can appreciate that. I think this gave me a better view that will help me work with veterans (SW1, para 5).

Lastly, this social work intern appreciated the knowledge she gained from her interdisciplinary care team. She acknowledged "This experience helped me get a realistic idea of the difficult cases I may work on when I work in a palliative care setting" (SW19). The team placement allowed learning for her future work. She concluded, "I think I need to be exposed to more cases like that so I can become more comfortable with things like talking to families about leaving their children behind" (SW19).

Subtheme 1b: Learning lessons: Being a chaplain. The nature of working on an interdisciplinary care team considered how chaplain interns and social work interns responded to the environment of patient, family, and team members during their interdisciplinary palliative care experience. The interdisciplinary placement and reflection upon it stimulated thoughts within the chaplain interns about their behaviors and how they conducted themselves as spiritual and psychosocial experts. In the excerpt below, a chaplain intern reflected upon the way he was touched by the relationship of a patient and her husband. It also indicated that he gained familiarity as the pastoral leader for the team when there was disagreement on her treatment. He noted,

I was personally affected by this family and the love and respect that existed between the patient and her husband. I was also gratified by the way I was included by the team to give my assessments of the family's spiritual and emotional health, to provide comfort to the family and even support the other team members during a conflict between physicians (CH18, para. 8). 
Learning about being a chaplain intern included self-knowledge, which could impact pastoral care. In this selection, a chaplain intern wrote about a self-discovery that came from caring for a family which was like his own. He observed the following new awareness about himself in this passage:

This chaplain visit has brought to my attention that some patient care interactions I have will remind me of my family and our own personal medical struggles we have experienced. When this occurs, I need to be aware not to push my own agenda (CH4, para. 8).

Another instance of learning about oneself as a chaplain was illustrated in a chaplain intern's reflective writing where she described she would rather respond to a need (e.g., lead a prayer) than simply be present (e.g., sit to keep family company; CH9). The nature of working on an interdisciplinary care team for chaplain interns involved providing pastoral care for non-communicative patients. A chaplain intern reviewed her patient's chart and discovered she was a Baptist and had a Do Not Resuscitate (DNR) code status. The chaplain intern explained how she concentrated on the spirituality of the patient, and this had increased her level of compassion for the patient and the family (CH13). The chaplain intern described how she gave comfort to her non-verbal patient in this selection:

We made much eye contact and tears formed in her eyes when I sang a couple of verses of "Jesus Loves Me." She has a close relationship with God and she was very accepting of my visit and singing. She reached out for my hand afterwards and we held hands for a while. I prayed with this sweet woman with a gentle spirit (CH13, para. 7). 
Subtheme 1c: Learning lessons: Being a social worker. Social work interns observed how their mentors conducted themselves as they worked on interdisciplinary care teams and learned from what they saw. How to approach a tough discussion about how much time a patient had left to live was observed by one of the social work interns (SW3). The social work intern explained,

This experience touched me personally because it was the first time I had witnessed a conversation with a patient when they were informed that they would not live much longer due to their cancer. From a professional standpoint, I learned a great deal of how to approach such a difficult conversation (SW3).

Learning how to be a social worker was described as providing family counseling sessions for a family with an 8-year-old son, whose mother was dying from cancer. A social work intern provided the background of this case, "The patient and her husband have had conversations with him to explain that his mother is sick and is trying to get better. They also explained it was a sickness he could not catch from her" (SW5). The intern told how she and her supervisor were helping them: "My supervisor and I were able to assist the family in providing interventions as to involve their son further in the mother's treatment and normalize his feelings of uncertainty and confusion during this difficult time in the family's life" (SW5).

Another social work intern explained learning to be a social worker in palliative care means little time was available to deliver support. In this passage, the social work intern revealed how quickly a patient's status can change:

One patient who had been admitted the night before my observation and had not been seen by the social worker yet actually passed away while we were reviewing 
her chart and getting ready to see her. The limited time people usually spend in the in-patient hospice unit greatly limits the kind of interventions that can be done and the degree of rapport the social worker and other team members are able to build with the patient and the team (SW13, para. 7).

Critical learning occurred for one social work intern from her experience with her mentor and a suicidal patient (SW22). She described that the patient had been diagnosed with both esophageal and prostate cancer, neither of which is curable. His physician suggested he receive palliative care; however, the patient changed his mind and received chemotherapy treatments.

The patient expressed suicidal ideation to the nurse practitioner, who referred him to the social worker. A session with the social worker occurred during the patient's lengthy chemotherapy treatment session. The intern observed how the social worker discussed the patient's emotions, coping abilities, and made a plan to remove a gun from his home (SW22).

The intern learned by watching how her mentor handled the desperation expressed by her patient. The intern described the encounter:

My experience with this patient was overwhelming. I have never worked with a patient that was so vocal about their suicidal ideation and who had a plan to commit suicide. I learned it is important to talk to the patient's family in order to discover the patient's support system. He did not seem to think that he had much of a support system, when in reality, he had few supports, they were apparent in his life (SW22, para. 8). 
Clearly, this passage reveals the deep feelings the social work intern experienced as she reflected upon the hopelessness of the patient.

The theme of Learning Lessons described what knowledge the interns gained as a result of their interdisciplinary palliative care placement. The interns learned what is important to interdisciplinary teamwork and ways the team influenced them. The interns' feelings about providing care as part of the interdisciplinary team was also located in this theme.

\section{Theme 2: Disappointment}

The second theme, Disappointment, is related to communication failures. Problematic interactions with other teams, team members, clinicians, and/or family members may cause a collapse in otherwise beneficial communications. Chaplain interns and social work interns witnessed disappointing collaborations or missed opportunities where patient care suffered, and may have led to more, perhaps even unnecessary, complications for the patient or the family.

Communication breakdowns occur in healthcare, which impact patient trust with providers and their ability to make decisions (Mazor et al., 2012). In several instances from the data, chaplain interns saw consultations not go as hoped for the patients and other issues of care turn into disappointments. For example, an intern wrote about an instance where one of the palliative care patients was losing brain functioning and the palliative care team worked to secure an inpatient hospice bed or placement in a nursing home. The interdisciplinary team faced a complication in care when the patient's primary oncologist disagreed with his treatment plans. The chaplain intern noted, "The primary oncologist for this patient felt that the efforts of the palliative care team amounted to 
giving up" (CH5). Another chaplain intern observed a lack of communication where "tests were scheduled, then cancelled once lab results were reviewed" (CH11). These miscommunications caused confusion for the teams and the patients.

Social work interns also witnessed communication breakdowns as they worked on interdisciplinary palliative care teams. At times, patient treatment is effective until another medical service or shift of personnel takes place. When a communication failure occurs, the interdisciplinary team attempts to correct the situation; however, the clinical relationship is often damaged. The excerpts below were the best examples of this theme.

In the case that follows, the family had reached the difficult decision to conduct a compassionate extubation (i.e., withdrawal of mechanical ventilation at end-of-life with death as an expected outcome; Youngwerth et al., 2015). The social work intern watched as the interdisciplinary team supported the family as they made the decision to remove life-support from their loved one. The interdisciplinary team had prepared the family for the patient's death. The family appeared to be content, even relieved, that the decision had been made. Respiratory therapy was contacted to extubate the patient, which occurred without the interdisciplinary team. The intern explained:

I was not present at the extubation. This occurred while the team members were having lunch. I was very disappointed the team was not present. I felt there was miscommunication on the part of respiratory therapy team. They did not contact the team to tell them they were ready to withdraw the ventilation tube. I felt the palliative care team should have been present because of their involvement with the family and the patient. I wondered if the lack of presence of the palliative care team could have been interpreted as not caring (SW4, para. 6). 
In another instance, a patient with end stage renal disease (i.e., the last stage of kidney disease) evidently was ready to stop curative treatments so she could enjoy her remaining time without experiencing the side effects. The chaplain intern described her encounter with the patient:

The patient had just asked her physician for a consult with the palliative care team. When I asked about her decision [to stop treatment], the patient stated very clearly she had been thinking about this for several months, praying about it, and talking with her priest about the spiritual dimension of contemplating the end of her life. For three months she had been examining the quality of her life, hoping for something different for the time she had left. Her husband said although he didn't want to think about her dying, he fully supported her decision. At that moment, the nurse reentered the room and shared a message from the doctor. The patient would not be given a consultation with the palliative care team until she underwent a full psychiatric evaluation. The nurse left the room, and the patient asked the chaplain intern, “Isn't it my decision? Don't I get to have any say about what happens to my body between now and the time that I die?" (CH24, paras. 2 and 3).

The patient in the above excerpt lost trust in the relationship with her physician when she did not receive a palliative care consultation. Her husband supported her decision for palliative care and she had also asked her priest if she were committing a sin if she discontinued treatment. Her physician never allowed a palliative care consultation. The chaplain intern described the treatment the patient received: "I believe she received wonderful medical care, but not the care she wanted or needed" (CH24). 
An additional instance of a failure in communication, which led to an interruption in care, was illustrated by another social work intern. The patient had a high level of pain and required collaboration with both the palliative care physician and psychologist to relieve his discomfort and restlessness. According to the intern, "The following day we learned that the patient and his son had a rough night because the second shift staff had ignored his dad. He felt that because his dad was dying, the staff didn't want to deal with him and made him wait too long for care" (SW23).

Although the interdisciplinary care team in this case had effectively managed the patient's needs during the day, the evening staff needed to improve their efforts in caring for him. The social work intern wrote the palliative care physician and psychologist were frustrated by this news. The patient's son was disappointed with the lack of care from the night shift for his dad. Following this incident, the interdisciplinary team suggested the patient be moved to another floor where he would be more likely to receive better palliative care.

Physician-to-physician communication breakdown was problematic. In an example of physician-to-physician disagreement, the palliative care physician and a chaplain intern met with a member of the oncology team who was concerned the patient's care goals had changed from full intensive care unit protocols to palliative care. The oncologist suggested to the palliative care physician that the patient "was not dying from cancer, but from pain medication she was being given" $(\mathrm{CH} 18)$.

This exchange caused tension between the two physicians. The implication from the oncologist was that the team had not done everything possible to help the patient live. 
The palliative care physician and the oncologist agreed the collapse in communication had occurred as a result of incorrect information given to the oncologist from a nurse.

Physician-to-family communication could be challenging, as revealed in this chaplain intern's experience. The patient's main physicians did not meet with the team. The chaplain intern explained another instance of miscommunication, where two physicians, a surgeon and an oncologist, gave conflicting information to the patient's family. The surgeon was optimistic about treatment and the oncologist was more guarded. The intern wrote these "conflicting messages were incredibly frustrating to the family" (CH22). The team was put in the position of dealing with inconsistent medical information put forth by the physicians who never met with the team members. The decision-making abilities of the patient and family were affected by contradictory communication.

Communication failures can also happen within the interdisciplinary care team. For example, one social work intern described a patient who was dealing with multiple brain tumors and needed to be discharged to an appropriate palliative care facility. Poor communication with the patient's physicians hampered the placement efforts of the interdisciplinary team. The social work intern described how the patient's care could have been improved. She explained:

The patient's family's care could have been improved if the doctors communicated more with the nurses and the social workers. It would have been very helpful if the neurologist discussed the patient's condition at length with the social worker and chaplain so they would have a better understanding of treatment options and care options. The interdisciplinary team may have learned more about 
the patient and her family if everyone discussed what they observed and did for the patient. Overall, having rounds each week can greatly benefit the patients who are entering palliative care, but this was not done for this particular patient (SW11, para. 7).

The intern wrote she gained insight from this case, especially about the importance of communication in an interdisciplinary team. The team members wanted to give the patient the best care options available and were disappointed they did not receive enough information from the patient's doctors to do so.

Lastly, another case demonstrated written communication failure from the patient's chart to a referral agency. The patient in this situation had incurable stomach disease and used feeding tubes throughout her life; however, she stated being in constant pain. She was a former hospice nurse and desired hospice care to be comfortable for the remainder of her life. The hospice agency nurse determined the patient had denied use of a feeding tube while in the hospital, which went against their criteria for admission. The social work intern wrote:

Shockingly, the patient had not been on a feeding tube or any other nutrient receiving system since she had been in the hospital. We are not sure what the hospice nurse saw that we did not. The social worker called again and stated the patient was not on any feeding system. She was re-evaluated the next day and accepted as a Hosparus patient (SW16, para. 3).

In this instance, the social worker called hospice and requested another evaluation, which resolved the patient's disappointment. 
The nature of working on an interdisciplinary palliative care team for chaplain interns is learning how to provide pastoral care to the patient, the family, and the interdisciplinary team, especially in situations where communication breaks down or things do not go as hoped or expected. Similarly, the nature of working on an interdisciplinary palliative care team for social work interns is learning how to give emotional support to the patient, the family, and the interdisciplinary team, specifically when trust has been lost as a result of a collapse in communication. No amount of study can adequately or completely prepare the chaplain interns or social work interns for the unpredictability of disease and events which occur to patients who are at the end of their lives. Participating on an interdisciplinary care team provides the interns with real life experiences and allows them many opportunities to learn about psychosocial-spiritual care and communication breakdowns.

\section{Theme 3: Being There}

Being There were the behaviors of the chaplain interns and the social work interns as present With the Team and For the Patient and Family. Being There: With the Team is defined as participating in interdisciplinary team matters (e.g., presenting spiritual assessments, providing cultural information, adding information to the patient's plan of care). The Being There: With the Team subtheme describes the effect of working as a member of the interdisciplinary care team for both groups of interns. The Being There: For the Patient and Family subtheme is defined as interactions with the patient or the patient's family as provided by the interns. Chaplain interns often provide pastoral support for the patient as they wrestle with their faith at the end-of-life or pray with the 
family as they ask for spiritual guidance to make a difficult medical decision. Social workers intervened about housing placements or emotional needs at the end-of-life.

Subtheme 3a: Being there: With the team. The chaplain interns were physically present at their interdisciplinary team placements and participated as active team members. These interdisciplinary team experiences formed the theme and subtheme of Being There: With the Team. The interdisciplinary team had regular formal interdisciplinary team meetings, where all members of disciplines put forth their assessments to form the comprehensive plan of care for the patient.

Outside the formal interdisciplinary team meetings, the chaplain interns communicated with the patient, the patient's family, and other team members. These informal informational contacts were also used to update the patient's plan of care. The interdisciplinary care team is the structure for providing comprehensive palliative care to the patient and the team itself creates experiences for the chaplain interns. The following selections were characteristic of the chaplain intern experiences.

In this excerpt, during a chaplaincy visit, the chaplain intern reported what the patient said regarding ending her treatments: "She felt she needed her children's permission to die, namely her two daughters whom she believed were likely to take news of her death the hardest" (CH11). The chaplain intern explained after collaborating with the team, the nurses, social worker, and chaplain, all encouraged the patient to voice her wish to end treatment to her family. The chaplain intern recalled how this situation was resolved: "After many tears, the patient was told by both her daughters that it was okay to go home to be with God" (CH11). This example demonstrated how the interdisciplinary palliative care team worked together to educate the patient so she could inform her family 
that she was ready to stop curative treatments. Each discipline supported the patient's goal to receive palliative care.

The following illustrative passages were the best instances of this theme from the social work interns. One of the social worker interns worked with a patient who received Hosparus (e.g., the local hospice agency) home visits. This patient was a non-English speaker and all communication was translated by her adult daughter. This family had very limited financial resources and their main concern for the family was to keep the heat on in their home. There were no other family members in the United States and the patient's goal was to return to Mexico to die.

Regarding interdisciplinary treatment for this patient, the social work intern stated, "The team provided quality hospice care and worked on this case very well. The overall well-being of her care was thoroughly discussed during the biweekly interdisciplinary team meetings" (SW2). The social work intern described her work with the family in this passage. She wrote:

There were numerous conversations held between the family and the IDT in regards to helping the family realize that the patient's cancer was only going to continue progressing and she would get weaker. The patient had expressed to her daughters that she would like to return to Mexico to die, and the IDT was on board with helping the family carry out these wishes. However, the family struggled to find payment for the plane tickets, and the social worker and I reached out to numerous churches and organizations for donations (SW2, para. 4). 
Some monies were gathered for the patient's trip to Mexico; however, the hospice nurse feared the patient was not well enough to make the trip and would die on the airplane, so she did not go to Mexico. The intern observed:

Helping the family understand that the trip could not take place was another struggle in itself, as the family had still not come to terms with the progression of the disease. The team worked together on this case very well, and the overall wellbeing of her care was thoroughly discussed during biweekly IDT meetings. The team always addressed the patient and family with empathy and comforted them during their multiple visits (SW2, para. 4).

Ethnicity and language differences were considered by the interdisciplinary team. The social work intern continued, "I also noted the team worked very hard to ensure the family understood the prognosis and did very well with meeting the clients where they were and explained it in a manner that was understandable for a different culture" (SW2). The social work intern experienced helpful interdisciplinary team interactions and learned from their example how to provide care. She concluded with this excerpt:

I learned a great deal of how to approach difficult, end of life conversations and to truly help families understand the reality of their loved ones' diagnosis. This family required multiple visits from the team to ensure that the best care was being provided at all times, and I learned that when dealing with end of life issues, there are so many other issues that arise associated with end of life that can be just as difficult. A positive observation made was that the team members collaborated very well with each other and would often talk to each other multiple times a day about this particular case (SW2, para. 5). 
Collaboration and constant communication were demonstrated by this interdisciplinary team. The intern gained a strong interdisciplinary palliative care team experience by being there, with the team.

Participating on an effective interdisciplinary team was a beneficial way to learn about team dynamics. Working as a member of an interdisciplinary team can also positively influence the chaplain intern in future work. One chaplain intern reflected, "This experience gave me the opportunity to see firsthand how the interdisciplinary team can work effectively together to give holistic care" (CH8). He noted he felt honored and humbled to be part of this team, adding "The impact of this experience encourages me to be a team builder and team player as a healthcare provider" $(\mathrm{CH} 8)$.

In another case illustrative of effective team work, the social work intern described the health condition of a patient who was from Pikeville, Kentucky. He was admitted for surgery during which the surgeon discovered colon cancer had spread throughout his body. After surgery, the palliative care team was consulted for pain management and to plan for the patient's return to Pikeville. Throughout his hospital stay, the social work intern observed "The team provided quality palliative care; the psychologist met with the patient and his mother to address the patient's anxiety and his panic attacks" (SW3).

Another chaplain intern reported a positive experience regarding her interdisciplinary care team placement. The patient in this case had entered palliative care because his prostate cancer was no longer treatable. He was struggling to care for himself as well as maintain his home. The chaplain intern observed the interdisciplinary team 
work together to accomplish the patient's pain management, future living arrangement, and complete a living will and advance directives. She saw how the patient enjoyed being involved in the decision making process and how he recognized value from his input (CH23).

The chaplain intern reflected upon team communication regarding plan of care development:

The plan of care was developed by the interdisciplinary team and assessments were made from each team member. Each member brought important information and insight to the care plan. Overall, the communication was key towards an adequate plan and we were intentional about meeting together in one space at one time with the patient and his family to discuss a plan of action and any other concerns (CH23, para. 5).

The chaplain intern summarized her interdisciplinary team experience. She saw the interdisciplinary team collectively work for the patient's goals, using their unique training and skills.

Another social work intern noted the interdisciplinary team care given to an elderly patient and her husband. While the interdisciplinary team have rounds together twice a week where patient needs are formally discussed, this social work intern saw numerous instances of informal sharing within the team. She wrote:

There seems to be a lot of communication among the various team members outside the rounds. Frequently, after visiting with a patient, if the social worker had noticed something new or gained some new information, she would stop by [sic] the nurse, doctor, or chaplain and discuss it with them (SW13, para. 4). 
The experience of being with the team provided additional learning about interdisciplinary team communication for the chaplain interns. While they were on the interdisciplinary palliative care team, the chaplain interns documented instances where they learned about effective team communication which was given to the family. Truthful and straightforward information from the team was given to the families in the following passages.

Two chaplain interns remarked about the need for direct communication presented by the team when plans were discussed with family members. "Open communication is important between family members and chaplains can help facilitate it" (CH14) reflected one chaplain intern. Another chaplain intern considered the truthful communication offered by the team members to the patient's daughter:

I felt the team did a good job letting the patient's daughter know what was going on in the process of her mother's death. We were careful to respect the daughter's emotions, but also were very honest with her about her mom's condition and what was ahead (CH15, para.6).

The chaplain interns experienced how being part of the team demonstrated caring and support to the patient and family. Chaplain interns saw how they promoted open discussions with family members. When a relationship exists between the interdisciplinary team and the family, honest communication can occur from the team to the family.

The social work interns gained more team experiences when they worked with the 
team. This example from a social work intern's observations of multiple instances of teamwork showed how the team supports the patient and family. The social work intern described team assistance for this patient:

The team made the patient feel like a priority, while still managing multiple other patients and making them feel the same way. The communication with multiple teams was definitely a benefit, as well as the team communicating with Mr. W.'s doctor in his hometown. Each team member made a point to make the transition back home as easy as possible and there were so many positive experiences I took away from this rotation (SW3, para. 4).

Subtheme 3b: Being there: For the patient and family. Being There: For the

Patient and Family describes what the interns do to support their patients and families. At times, chaplains must step in where they are needed most, which may be to provide more than pastoral care and social work interns saw the many roles they take on as they provide care. These behaviors define what it means to be interdisciplinary. The following selection was the best chaplain intern example of the theme.

In this case, the unit chaplain (i.e., the chaplain intern's mentor) demonstrated to the chaplain intern how to give emotional support and exchange information for a family whose loved one was in an emergency situation. The unit chaplain delivered key communication between the medical professionals and the patient's family during a resuscitation code. The patient was resuscitated; however, afterwards, it was determined his prognosis was very poor. The code response team suggested a meeting with the palliative care team. The chaplain intern was also able to participate in the palliative care meeting later that same day $(\mathrm{CH} 2)$. 
In this scenario, the chaplain intern observed how being a team member meant providing critical understandable medical information for the family of a patient. The family had seen their loved one nearly die in the morning, then be referred to palliative care later the same day. The chaplain intern wrote both encounters "really underscored for me the importance of palliative care in making sure that both patient and family care are provided" $(\mathrm{CH} 2)$.

The unit chaplain supported the family by providing them with medical information during the resuscitation, which was vital. At that moment, the chaplain intern watched as the unit chaplain was the voice of the resuscitation team. When the palliative care referral was made, the chaplain intern saw the unit chaplain give emotional support to the family as they were informed about the ways palliative care could be helpful for the patient's future care $(\mathrm{CH} 2)$.

The next examples were representative of how the social work interns provided support to the patient and family during their placements. One case involves an elderly man who had complications from head and neck cancer who communicated his wishes to begin palliative care (SW23). The interdisciplinary palliative care team worked with him to manage his pain and establish goals of care. The patient and his son decided together that he would no longer use a ventilator or feeding tube, and asked for a DNR (i.e., Do Not Resuscitate) order to be placed in his chart.

The social work intern reflected on how this patient knew he was ready for palliative care: "He had decided several months before that he would end life-prolonging measures when he could no longer be at home and enjoy sitting on his front porch" 
(SW23). The patient's son was concerned about making sure his father was comfortable and the intern noted, "The psychologist and physician validated the son's feelings and reassured him they were here to also support him" (SW23).

Sometimes, interns had patient experiences that touched them at a personal level. In one instance, a social work intern described the effect the patient's husband had on her: "The love that the husband had for his wife and the extreme amount of support and devotion he provided was truly moving" (SW21). She also wrote it was hard for her to not to picture her family members who had fought cancer as she worked with this patient and family.

Palliative care experiences had powerful influence on the interns. The patient of one social work intern was a 33-year-old wife and mother who suffered from advanced metastatic breast cancer. This couple had an 8-year-old son who required assistance and communication from the team to help understand his mother would not get well. Care was also given to the patient and her husband throughout her inpatient stay as they journeyed to the end of her life. The social work intern described what she gained as a result of working with this patient:

This experience with the patient and her family has motivated me to go above and beyond to get to know patients and be an advocate for them. It is my job as a social worker in the health field to be a voice, supporter, motivator, and educator to patients with the aid of a cohesive palliative care team, with an end goal of putting the patient first (SW5, para. 7).

The social work interns wrote about their interdisciplinary team experiences when they were there for the patient and family. At times, the interns revealed being with the 
patient and family reminded them of the medical struggles from their own families, or they saw how to provide support for a patient's decision to allow for natural death. These interactions motivated the social work interns work within an interdisciplinary team and be advocates for their future patients.

\section{Theme 4: Dealing with Family Matters}

The theme of Dealing with Family Matters, describes the dynamic interaction of patient and family. Family environments are usually not subject to change in the short period of time the patient is in the hospital or before they die; however, the background of the family affects patient care. The patient and family are considered to be the "unit of care" and a focus for the interdisciplinary care team. Within the plan of care created by the interdisciplinary care team, the patient and the family are valued members and are expected to have input.

Two subthemes illustrated this theme. Dealing with Family Matters: Complicating Factors examined negative family interaction which was harmful and Dealing with Family Matters: Simplifying Factors illustrated positive qualities within the family that were helpful. The passages which follow were the best examples of intern palliative care learning.

Subtheme 4a: Dealing with family matters: Complicating factors. Although the patient's needs are very important, the family situation and dynamics are also considered in the plan of care. The family environment can exert influence on the patient at the end-of-life. The chaplain interns frequently encountered difficult family dynamics that caused problems for the family during an already stressful situation. The chaplain interns found ways to give support when families were not in agreement on care. 
The chaplain intern for this patient described her to have dementia and renal failure, who was expected to live for several weeks. The patient was portrayed as easily agitated, unable to recognize her family, and at risk for a fall. With these caregiving problems, her family was incapable of providing for her at home (CH10).

The plan of care meeting with the family was explained by a chaplain intern. The family was in disagreement over her treatment. The patient's daughter wanted her sedated and forced to undergo dialysis. The daughter wanted to make all decisions for her mother's care, because she was angry with her mother's husband and did not trust him with decisions about her care. This absence of trust in the family environment caused tension between her and the husband.

The daughter stated she just could not make the decision to let her mother die. During a family meeting with the team, the chaplain intern asked if there was something that would help her feel better about making the decision for palliative care. Help for the daughter was given by the chaplain intern, who wrote:

Would she like to pray? To talk to someone? To talk to the patient even, so it didn't feel like a secret...just to tell her she was trying to make the best decisions for her out of love and her best interest. We talked about what the patient valued in life, and based on that, what would she want her final days to look like? (CH10).

The husband did not want the patient to be in pain and made the decision the daughter could not make, which was to begin hospice care. They agreed to have palliative care follow the patient until an inpatient hospice bed was available. The chaplain intern ended by writing, "I thought everyone present contributed in a positive way. Educating 
the daughter to the reality of the situation was paramount for her to find peace in the decision" (CH10).

Participation on an interdisciplinary palliative care team brought forth multiple instances for the social work interns to work with patients' families. Social work interns also often encountered family forces that worsened the existing complex medical decision making. In the following instance, this social work intern examined how support was offered when family circumstances were bleak.

The patient in this case lived with his son and daughter-in-law, who were also his caregivers (SW1). In addition to the patient, the son and his wife provided care for her elderly parents, who were in poor health. The patient's son was recovering from a stroke that had occurred a year ago. The patient had been receiving in-home hospice care for the past month and was admitted to the hospital to manage his pain and plan for his current state of physical decline.

The social work intern and the social worker in this situation explored placement options for the patient at his discharge. The caregiving burden was described by the intern as very intense and the son and daughter-in-law were unable adequately provide for the patient. The social worker and the intern explored nursing home options for the patient; however, the patient did not qualify for Medicaid because he has too many assets to meet subsidy requirements (SW1).

The patient's main asset was property in Southern Indiana, but it was in disrepair and therefore, difficult to sell. Unfortunately, the funds to pay for a private nursing home did not exist. The social work intern remarked about this challenging family situation: 
This experience had an impact on my future as a healthcare provider insofar that it helped me understand there will be cases without good solutions. I still need to try and work hard to find the best solution possible, I learned this is just out of my control and I need to be okay with that (SW1, para. 6).

Although the son was willing to take his father home from the hospital, he also indicated caregiving for his father put enormous strain on him. The caregiving burden for one couple to provide for three elderly parents was great. Obviously, family environments like these can be trying for social workers to find workable resolutions.

Likewise, chaplain interns also discovered patients and their families face many difficulties as they navigate the healthcare system. Complicated family dynamics can aggravate already difficult care decisions. This case involved a patient who was hospitalized with late stage brain cancer (CH5).

According to a chaplain intern, the patient had previous surgeries, which left him severely physically disfigured and functioning like a child rather than an adult. The patient's sister, described as having below-average intelligence, was the caregiver for the patient and his parents. During his hospital stay, the patient's mother died. The patient's father humiliated him and played cruel jokes on him as he lost cognitive ability and awareness [over time] as a result of his late stage brain cancer (CH5).

The chaplain intern found the patient to be interested in watching television and unable to hold on to more than very few details of a conversation before getting distracted. The main problem with communication, the chaplain intern realized, was the care team had failed to see the whole person that was the patient. In the following passage, the chaplain intern wrote: 
While the nursing staff and doctors discussed various medical needs that he had, and his sister worked with the social worker and chaplain [intern] to figure out where he was going to go next, it struck me in retrospect that we had failed to identify the patient's humanity. Because he would neither respond when things were discussed with him, nor remember what had been said, we didn't really interact with him that much (CH5, para. 6).

The chaplain intern continued, in the following statement about the person in the center of the discussion, the patient, and stated:

What I learned from this experience that I will carry forward to the rest of my pastoral care is that it's important to always engage a patient as a human being, even if they don't know you are doing it. It was also an opportunity to reflect, again, on the complexity of family situations. The patient wasn't just sick. He was sick in the context of a dying mother, an abusive father, and a sister who was not well-equipped to take care of any of them. It is important for everyone on a team to remember to look at the big picture, then to take a step forward and re-engage with the very real humanity of the patients we care for ( $\mathrm{CH} 5$, para. 7).

Family relationships can also become demanding for social workers and the patient when agreement on treatment is desired. The patient had incurable stomach disease and suffered from it for many years. She sought hospice care and wanted her physician and her husband to support her decision.

The social work intern noted the nurses, social worker, and patient had great communication (SW16). However, things did not work out the way the patient wanted. She was not only turned down for hospice care, but she also did not have the support of 
her physician for hospice care. Her doctor tried to send her to the psychiatric unit because she could have depression, which was interfering with her judgment. She was assessed again the next day by Hosparus and accepted as a patient. The social work intern described the patient's encounter with her husband:

The husband did not support his wife's decision for hospice care; the patient asked the social worker to contact him and explain to him what was going on now that Hosparus had accepted her. The husband was not accepting of the information even from the social worker. He accused his wife of seeking pain medications rather than comfort measures. He agreed with the doctor that she be admitted to the psychiatric unit. Thankfully, the patient was deemed competent and made her own decision to follow through with Hosparus despite her husband's wishes (SW16, para. 6).

The social worker communicated between the patient and her husband. This demanding family communication had an impact on the social work intern. She described how she felt:

This experience touched me deeply to the point of fighting back tears in the patient's room listening to her story and how the professionals in the hospital were not all listening to her. My heart broke for her and I wanted her to have everything that she wanted with Hosparus (SW16, para. 8).

Subtheme 4b: Dealing with family matters: Simplifying factors. Preplanning and discussions about palliative care can give spouses and families comfort when end-oflife choices must be made. Knowing the wishes of the patient are carried out can be reassuring to the family. One instance of family unity and support after a patient had a 
severe stroke from which he could not recover was illustrated by this chaplain intern (CH6):

The patient's wife and I talked about palliative care and she told me she was at peace with her decision. She knows palliative care is the right way to go and her husband will be made comfortable. She was aware of the benefits of palliative care. She and her husband had discussed his wishes months ago (CH6, para. 4). Experiences from an interdisciplinary palliative care team offered many opportunities for the social work interns to work with patients' families. Family dynamics can support treatment decisions families often make for their loved ones. In this subtheme, social work interns saw how families with healthy patterns of interaction noticeably improved their ability to make decisions.

Caregiving needs can quickly change, as was the issue in this case. The social work intern described loving family caregiving support offered to an elderly patient whose caregiving requirements had drastically changed since he was admitted with a hip fracture one week ago (SW8). When family harmony exists, planning can be easier.

A plan of care was devised for this palliative patient with the interdisciplinary team and the patient's family. The family was very willing and financially able to provide care. The patient's home was near his son and daughter-in-law, as well as one of his grandchildren. The social work intern remarked, "I was rewarded with the opportunity to see how a family takes compassionate action to care for their loved one" (SW8).

The chaplain intern wrote that preplanning and discussion about palliative care, as well as the prayer and faith life of the family, had a positive impact on him. In the following instance, the patient had a fall, which led to paralysis, then quickly to palliative 
care after he was found not to be an operative candidate. The patient's family voiced their desire to keep him comfortable and understood he would never recover from his injury. The interdisciplinary team worked to achieve the family's goal for the patient, which was a peaceful, comfortable death. The chaplain intern described the family's support for their patriarch, "It was apparent the family was close in heart and mind. The sons honored their dad in the way they honored his wishes to die naturally" $(\mathrm{CH} 21)$.

Another case with helpful family factors was documented by this social work intern. She wrote,

I was impressed with the strengths of the patient and family. They were respectful of the patient's wishes and continued to work to achieve his goals of care. Facing end-of-life together, the family was incredibly supportive and this was touching to witness (SW6).

A cooperative family environment for the dying patient can make things easier for the patient as they ease into the final stage of life. When families respect and uphold their loved one's wishes for treatment or to begin comfort care, harmony is experienced for all.

\section{Theme 5: Making Hard Decisions}

The fifth theme that emerged from the data was Making Hard Decisions. At the end-of-life, families are often asked to make difficult choices about treatment for their loved one. These medical options can be very difficult to understand, especially during times of great stress. The outcomes are usually grim and families require education and support in order to make care choices for loved ones. Examples from both groups of interns were reviewed. 
Patients may arrive at the hospital and imminent death is not expected; however, at times, the decline of the patient can be very rapid. The family has little time to prepare for death in cases such as these. At other times, the patient may be non-communicative, or on a ventilator, when decisions need to be made. The chaplain interns explained the outcomes of treatment choices and emotionally support the family throughout the end-oflife decision-making process. The passages below were representative of nearly every chaplain intern's experience with difficult treatment choices.

Seventeen chaplain interns wrote reflections about patients who were ventilator dependent or unable to communicate. Six chaplain interns $(\mathrm{CH} 7, \mathrm{CH} 8, \mathrm{CH} 17, \mathrm{CH} 19$, $\mathrm{CH} 20, \mathrm{CH} 21)$ wrote about cases where patients were on ventilators; $11(\mathrm{CH} 1, \mathrm{CH} 2, \mathrm{CH} 3$, CH6, CH9, CH10, $\mathrm{CH} 11, \mathrm{CH} 13, \mathrm{CH} 15, \mathrm{CH} 16, \mathrm{CH} 18)$ others had patients who were unable to speak, due to medication, fatigue, or advancing disease. All of these patients were non-communicative and therefore unable to direct their own care. Families were left to choose what course to take for their loved ones and chaplain interns accompanied them as they decided and once action was taken.

Typically, the patients on ventilators were compassionately extubated. The other non-communicative patients relied upon earlier care discussions with their families to guide treatment, or began comfort care as part of the interdisciplinary plan of care created during their stay in the hospital. Chaplain interns provided support for the loved ones in these tough situations.

One chaplain intern described a compassionate extubation, when death did not quickly follow. The patient was extubated on a Saturday morning, and the chaplain was paged to support the family after the ventilator was removed. A large group of family had 
surrounded the patient's bed and the chaplain intern interpreted the mood in the room as subdued and slightly angry. These responses were attributed to the assumption the patient would die quickly after extubation (CH17).

The chaplain intern accompanied the family in their vigil and determined their mood lightened; however, they realized death would not come quickly. The chaplain intern checked back with them twice that afternoon, before receiving a page that the patient had died, 12 hours after extubation. The chaplain intern in this case wondered what could have been done to further support the family or to have made the patient's end-of-life experience more meaningful for them. He described that he felt negligent for not telling the family to care for themselves during the long day and wondered, "Had palliative care addressed their exhaustion?" (CH17).

Another patient on a ventilator received comfort care as she was unable to see or talk. Her family required a great deal of pastoral care, described as "given by chaplains, two or three times per day" (CH19). During a prayer for family and friends of the patient led by the chaplain intern, the patient's daughter ran from the room, screaming and crying. The chaplain intern described what happened next: "I stopped the prayer, but one of the patient's relatives asked me to continue with the prayer. I finished the prayer and went outside the room. I saw the daughter surrounded by the staff comforting her" (CH19). This chaplain intern identified what she does for a family, which is to carry on a conversation and try to form the beginning of a relationship so they might feel comfortable to ask for prayer.

Several of the social work interns also had patients who were unable to communicate their wishes. The interdisciplinary care team was on hand to let the family 
know the expected course for their loved one. Options and choices were explained to the family by the team and support was also extended during the difficult time spent wrestling with what to do. The social work interns' thoughts, emotions, and feelings illustrate what they faced from life and death situations while they were on their placements. The most expressive passages that follow were used to illustrate their learning.

The patient of this social work intern was a 33-year-old father who was on a ventilator due to acute respiratory failure and multiple sites of cancer. He was unresponsive and the interdisciplinary palliative care team was called for consultation. Several weeks later, his physical condition had deteriorated and he was considered to be brain dead (SW4).

The social work intern said the interdisciplinary team had worked to provide the best care they could for the patient and his family. However, the patient was now brain dead, the result of a brain hemorrhage, and he would not recover. The social work intern described how the team educated the family about their choices:

The palliative care team I had worked with, the nurse and social workers had worked to provide the best care for this patient and his family. According to each one's specific role, the team had assured that the patient was not in pain, explained the finality of his condition, and listened to the individual family members as they shared their grief, tears, and loving, happy stories of their loved one. I thought the palliative care team worked as one, not only to provide the best care for the patient but also for the family (SW4, para. 6). 
The interdisciplinary team met the needs of the family who had the difficult decision to terminate the ventilator and allow their loved one to die. The social work intern expressed her final thoughts about this case by stating, "The support given to the family by the presence of the team was very beneficial to them. It let them know that people cared about them and understood what they were going through; that they were not alone" (SW4).

The chaplain interns expressed thoughts, emotions, and feelings about their cases in their reflective writings. One chaplain intern revealed an interdisciplinary palliative care experience, which left her changed (CH24). Her patient was described as exhibiting many symptoms common to end-stage renal disease and she had asked for a palliative care consultation.

The chaplain intern described that the nurse gave the patient the hospital's brochure on palliative care. Her husband also supported her decision for a palliative care consultation. Her nurse entered the patient's room and stated her physician would not allow a palliative care team consultation until she underwent a psychiatric evaluation, which greatly upset the patient. The chaplain intern in this case wrote "I value patient autonomy above all else, sometimes overly so" (CH24).

The patient's priest was also asked for his opinion on her desire to terminate curative care; he supported her decision and stated discontinuing treatment was not the same as ending life. This case was addressed at the ethics committee and dismissed, because the physician said he believed palliative care interventions would not have been helpful. In this excerpt, the chaplain intern concluded with the impact she felt from this event: 
I was profoundly moved by this experience. I felt the patient's needs—as a whole person — were being held hostage to a particular point of view. I have a clear sense that had we been able to meet as a team, with the family, that an outcome that supported the patient's broader concerns could have been addressed and met in ways that supported everyone. I believed I lacked the authority to question a doctor. I don't believe I would remain silent again ( $\mathrm{CH} 24$, para. 11). A patient's health status can quickly change when a traumatic event occurs. Caregiving needs become more intense when the family member is unable to care for himself any longer. This social work intern watched the interdisciplinary palliative care team help a family decide ways they could care for their 93-year-old father now in palliative care as the result of a hip fracture. The social work intern reacted to the rapid decline of this patient and writes, "This situation, for me personally, is frightening. The patient was out cutting his grass a couple of weeks ago; he was actively engaged in daily living before he broke his hip and became bed-bound" (SW8).

Another case spoke to a paradox in instances where patients cannot communicate their wishes or needs, and yet, all discussion was about these patients. The 48-year-old patient in this case was the mother of seven children, all of whom were under 18 years of age. The patient lost consciousness at work and was brought to the emergency room. Once admitted to the hospital, her condition seemed to improve. However, five days into her hospital stay, she became very ill and was intubated. She was no longer able to speak or move her arms or legs. It appeared as though this patient would not return to her normal life and might have a few weeks to live (CH20). 
A chaplain intern from this case expressed his thoughts when he wrote, "Although this situation is about the patient, we never get to talk to her or hear her own side of the story. She is not able to make conscious decision and movement. Yet whatever decision would be made is about her" $(\mathrm{CH} 20)$. He continued to wrestle with her situation and stated, "Her seeming imperceptible presence invites everyone involved in her care to be mindful of her wishes and dignity" (CH20). He continued by saying, "This situation highlights the somewhat high moral burden of responsibility caregivers, family, doctors, social workers, nurse, chaplain, etc. carry" (CH20). His thoughts about the interdisciplinary team's duty to the patient and family reminded one of the expectation and obligation for ethical practice when giving care to those at the end-of-life.

Working in palliative care demands a certain comfort level with dying patients. No one can predict the speed of one's death. Another social work intern described her work with two patients who did not achieve their goal of hospice care (SW17). This social work intern observed Hosparus evaluations for her interdisciplinary palliative care experience for hospitalized patients. She recounted her experience with two patients scheduled to leave the hospital for home. The first patient told her family while she remained alive she could make her own decisions and she chose a rehabilitation facility for her care. Her health quickly took a downward turn and she died before a Hosparus bed could be found for her (SW17).

The second patient from this social work intern's reflection was caught in family indecision. The patient's family could not make a quick decision about Hosparus care; however, after several days, they made a choice to use it. Before the patient could be 
interviewed by Hosparus, he died (SW17). The social work intern recalled the effect the death of the first patient had on her:

I do not know how I felt on either of those cases. I was completely dumbfounded. It was very difficult to learn that the patient passed away. I had to excuse myself from my work; I did not want to seem weak or let anybody see me cry (SW17, para 4).

She wrote about her feelings from the loss of the second patient, "I was walking by the room when the nurse said the patient had taken his last breath. It felt like the room was cold and empty. The feeling gave me chills as I walked back to the nurses' station" (SW17).

One social work intern saw the important contribution each discipline gave to the patient at the end-of-life. This patient had many long-standing trauma issues and family difficulties, which could not be fully resolved before he died. The social work intern believed the expertise of the entire interdisciplinary palliative care team would be needed in order to help him achieve a peaceful death (SW10).

Reflections from this social work intern spoke to the thoughts, beliefs, and feelings she experienced from working with this patient, "Personally I was touched overall by the patient's story and his interdisciplinary team because it was apparent that to offer him quality end-of-life care, all medical, social, and spiritual services would be needed" (SW10). Deeper insight was gained as the interdisciplinary team developed the patient's goals of care when she wrote, "It is always important to remember the client's values and goals are much different than our own and it is only ethical to respect them" (SW10). 
In summary, both groups of intern gained knowledge by being present on the interdisciplinary palliative care team. They learned how to be on a team from the team and how to relate to the patient and family as a member of the team instead of a single discipline, working alone. The team had an influence on the interns, which prepared them to be helpful members on future teams.

Family environment is important when the interns work with the patient and the family because long standing dynamics can affect treatment decisions. The context of some families were harmful, which made providing comprehensive care more difficult for the interdisciplinary team. Other family dynamics were helpful and eased patient concerns at the end-of-life.

Very often patients are unable to communicate their care wishes and rely on family members to make decisions about their treatment. The interns encountered families who faced making difficult treatment decisions for a loved one. This is the reality of working in palliative care.

Most of the chaplain interns experienced working on an interdisciplinary care team with non-communicative patients. These patients were kept alive by ventilators or very near death and could not participate in their own care decisions. Instances of care were provided to the family before and after ventilator removal by the chaplain interns. The weight of a making a hard decision for a family was not taken lightly by the chaplain interns.

The social work interns observed patients' physical abilities rapidly decrease as the result of trauma. Families have no preparation when a traumatic event lead to a much higher level of care for the patient, either emotionally or when they physically provide 
caregiving. It was described as frightening to witness the rapid decline of a patient who fell by one social work intern. The interns realized there was only so much that can be done to affect positive change in some situations.

Theme 6a: Intern as Existential Comforter. The final categories for the interns were represented by themes from their disciplines. The theme of Intern as Existential Comforter described the unique contributions made by the chaplain interns during their interdisciplinary team placement. The chaplain interns spent much of their time leading prayer, providing ministry of presence during vigils, or, in one instance, singing to a noncommunicative patient. Chaplain interns also provided pastoral care for the interdisciplinary care team members when issues arose, connected with outside clergy, or other organizations when needed for patients and their families.

Chaplain interns provided comfort to those at the end-of-life. They also worked with patients and families when existential issues arose when death was near. Chaplain intern roles on the interdisciplinary team provided support to the patient, the family, and the staff. The following examples were the best illustrations from the interns' reflections.

The patient in this situation was in the hospital with lung cancer. He lost his wife to cancer eight years earlier; he knew she suffered and he waited too long to call hospice. A chaplain intern related his interaction with the patient who was predicted to recover from his pneumonia and continue his chemotherapy treatments; however, his prognosis remained guarded.

The chaplain intern wrote the patient's first goal of care "was to get well and beat the cancer" (CH22). The interdisciplinary team honored this request, although in private, the team believed the patient was unwilling to fully acknowledge his illness. The chaplain 
intern described his private conversation with the patient: "He shared his fears about his fate but felt he had to trust in God to care for him and get him better. He knew he might not survive but felt that staying positive was the best way for him to cope right now" (CH22).

The chaplain intern described how he comforted this patient. He "helped the patient name a goal, which was to identify something that would tell him to shift his treatment away from curative measures and consult hospice" $(\mathrm{CH} 22)$. The patient decided he would consider hospice care when his chemotherapy became too painful. Although not a typical spiritual intervention (e.g., prayer), the patient was given time to consider his treatment options and the chaplain intern provided comforting support as the patient thoughtfully made his decision.

Not every patient's family shared caregiving responsibilities. The course of a patient with sepsis (i.e., an illness which is in response to an infection which may quickly become life-threatening) and dementia was detailed by a chaplain intern. The youngest daughter of the patient had been her live-in caregiver until the burden of her care became too great, when she asked for help from her older siblings. The caregiver thought the older siblings would allow their mother to live in their homes, thus making a sacrifice for the patient as she had done. However, the older siblings' decision for their mother's care was to place her in a nursing home, which made the youngest daughter resentful (CH16). The chaplain intern described providing comfort to this contentious family. The family was invited by the chaplain intern to talk about the patient. At this time, the chaplain intern wrote, "The family has been doing well sharing stories and beginning to 
share openly with one another about their feeling about their mother/grandmother" (CH16). The chaplain intern recounted how the family reacted at a later time:

On a follow-up visit, after sharing some more stories, we gathered around the bed again for a prayer and I invited each of them, if they felt comfortable, to share a memory or something they were feeling at that moment before we prayed. Each person shared something and we closed in prayer (CH16, para. 7).

The chaplain intern acknowledged the patient's hospital room was an area of relief for the family, where each member connected to the others by telling a story about their loved one. When death is near, tension within a quarrelsome family can escalate. The chaplain intern gave comfort to a family in conflict.

One chaplain intern described the care the patient received from the interdisciplinary team in scriptural terms. A chaplain intern viewed her pastoral training as a way she provided ministry to the team and assigned scriptural meaning to patient care given by the team. She described her pastoral care:

As the chaplain, not only was I able to serve the patient and his family with pastoral care services, but I also was able to minister to the entire palliative care team during our meetings and assign theological meaning to the importance behind coming together as a collective force to provide the best healthcare for our patient. It supports the spiritual principal and staple in the Christian faith to love and care for 'thy neighbor' (CH23, para. 7).

Chaplain roles on the interdisciplinary team included providing spiritual assessments and pastoral support to patients and families. However, chaplain interns often filled other roles for the patient and family. In this situation, choosing the funeral 
home for their loved one caused a high level of emotion within the family. The patient had grown up in a religious family; however, she had not attended church in decades, as described by the chaplain intern.

The chaplain intern told how she comforted the worried family: "When the patient died, I was able to answer the family's questions about funeral arrangements. I facilitated conversation amongst the family members about which funeral home to choose and what type of services to arrange" (CH1). This chaplain intern's modest act of handling the funeral arrangement helped reduce the family's level of stress.

Chaplain interns are also expected to provide pastoral care to the patient, the family, and to the staff (Common Standards for Professional Chaplaincy, 2006). One chaplain intern described the roles of the chaplain:

I have witnessed and learned that palliative care is involved in interacting with patients, families, and providers. The heart and actions of the palliative care team push me to be a caregiver who advocates for the patient and supports the clinical $\operatorname{staff}(\mathrm{CH} 3)$.

Theme 6b: Intern as Psychosocial Resourcer. The theme of Intern as Psychosocial Resourcer defined the distinctive behaviors and interactions associated with being a social worker. These were the discipline-specific roles expected of the social worker who is on an interdisciplinary team. Social work interventions and activities performed by the social workers for the interdisciplinary team can vary from conducting family sessions, helping complete advance directives, and/or find a higher level of care for patients upon discharge from the hospital. The theme of Intern as Psychosocial Resourcer is used to broadly describe the unique activities and roles reported by of social 
work interns. The descriptive passages that follow were the most illustrative from their placements.

In some instances, patients wanted someone to be with them when they die, but their spouse cannot fulfill this wish. This social work intern described an elderly patient who was expected to being active dying within the next few days. The patient said she did not want to die alone.

This patient's husband in this case was in poor health and attempted to be at her bedside most of the time; however, this situation was very difficult for him. The social work intern wrote, "The social worker has contacted a volunteer to be with the patient once she begins the dying process so she won't die alone" (SW13). The social work intern reflected on the importance of being with the patient when she wrote, "I learned that making sure people are comfortable can mean things like pouring a glass of water or just sitting with a patient---things that aren't typically regarded as social work interventions" (SW13).

Another social work intervention worked for consensus between the patient, family, and caregivers. The social work intern in this case saw how the patient regained her voice in her care, due in large part to the social worker's ability to bring all parties together on a plan of care. The patient was an elderly divorced mother of four adult children, all who lived out of state. She was unable to endure further surgery or procedures and was now under the care of hospice. One of her daughters was her medical surrogate and power of attorney. The patient was cared for by a close family friend (SW18). 
The social worker in this case handled the daughter's main concern about overmedication of the patient. She believed her mother developed a drug dependence to escape her dismal circumstances. The social work intern described how the social worker was the advocate for the patient. She described the intervention:

Communication was a key factor in this case. Some of the issues were resolved due to enhanced efforts on everyone's part to communicate their position. The daughter had the opportunity to speak with several members of the interdisciplinary team including the social worker, the doctor, and at least one nurse. The daughter and the caregiver were reassured the patient was receiving competent and compassionate care; it also seemed to ease some of their distress (SW18, para. 5).

One social work intern identified an instance where another type of support was provided. The social work intern described her patient as young father who was dying from colon cancer. The patient was depicted as "a very devout Christian and a minister at his church" (SW19). The social work intern described a visit where the patient “constantly talked about God and God's plan for him. He always explained to the medical staff who checked on him that he is at the mercy of God and if this is God's plan for him then he will accept it" (SW19).

The social work intern revealed it was difficult for her to talk to the patient about religion because she was Hindu. She wrote, When the patient would talk about God and Jesus, I politely agreed and affirmed his feelings about the matter. I did not engage in that topic too much because I was afraid of offending him. The chaplain had been contacted to see the patient 
and his family, but he liked to share his religious views with all medical and hospital staff who saw him (SW19, para. 9).

Each discipline has its own unique contribution to make to the team and with the patient and family. Chaplaincy is concerned with giving attention to the transcendent matters of all: patient, family, friends, and interdisciplinary team members. Chaplain interns experienced the types of roles expected of them during their palliative care clinical placement. Social work interns are expected to provide palliative care using assessment and intervention for the social and psychological wellbeing of the patient, family, friends, including caregivers, before and after death (Monroe, 2010). Social work intern role experiences included the varied aspects what they may encounter as future palliative care social workers.

\section{Similarities between Intern Groups}

The chaplain interns and the social work interns viewed their interdisciplinary palliative team placements, patients, and interventions through their discipline-specific roles; however, similarities existed between the groups. Both the chaplain interns and social work interns experienced effective communication within the interdisciplinary palliative care team. They also pondered useful instances where the interdisciplinary team's plan of care, developed with the patient and family, was helpful to the patient and family. All similar illustrations were used from both intern groups. 
Table 3.

Documented Similarities between Chaplain Interns and Social Work Interns

\begin{tabular}{|c|c|c|c|}
\hline Theme & Subtheme & $\begin{array}{l}\text { Chaplain } \\
\text { Interns } \\
(\mathrm{N}=24)\end{array}$ & $\begin{array}{l}\text { Social Work } \\
\text { Interns } \\
(\mathrm{N}=23)\end{array}$ \\
\hline \multirow{6}{*}{$\begin{array}{l}\text { Experiences: } \\
\text { Effective Communication with } \\
\text { the Team }\end{array}$} & Daily & 1 & 1 \\
\hline & Constant & 2 & 3 \\
\hline & Intentional & 2 & 1 \\
\hline & Effective & 1 & 2 \\
\hline & Excellent & 1 & 1 \\
\hline & Collaborative & 3 & 2 \\
\hline \multirow{3}{*}{$\begin{array}{l}\text { Experiences: } \\
\text { Effective Communication for the } \\
\text { Patient and Family }\end{array}$} & Helpful & 3 & 4 \\
\hline & Honest & 3 & 2 \\
\hline & Supportive & 5 & 2 \\
\hline $\begin{array}{l}\text { Behaviors: } \\
\text { Encouraging Communication }\end{array}$ & & 16 & 15 \\
\hline \multirow{2}{*}{$\begin{array}{l}\text { Feelings: } \\
\text { About Interdisciplinary Palliative } \\
\text { Care Teams }\end{array}$} & Important & 11 & 8 \\
\hline & $\begin{array}{l}\text { Care from each } \\
\text { discipline is essential }\end{array}$ & 4 & 4 \\
\hline
\end{tabular}

The data analysis used to answer this question required review of the Values Codes used for Initial Coding. The Values Codes were used to code chaplain intern and social work intern values, beliefs, and attitudes from their interdisciplinary palliative care team experience. Words and/or short phrases that were documented by both chaplain interns and social work interns were counted and used to construct the themes and subthemes to answer this research question.

The chaplain interns and the social work interns reflected upon the experience of the positive exchange of ideas between members of interdisciplinary palliative care teams. Effective communication with the interdisciplinary palliative care team was described as daily (CH1, SW9), constant (CH9, CH14, SW1, SW10, SW13), intentional (CH9, CH11, SW15), effective (CH18, SW2, SW5), excellent (CH19, SW8), and collaborative (CH9, CH10, CH18, SW2, SW6), by both the chaplain interns and the 
social work interns. Both groups of interns experienced similar positive and helpful within-team communication.

From their written reflections, the chaplain interns and social work interns also experienced similar positive interdisciplinary palliative care team communication with the patients and their families. The interns often used their discipline-specific activities with patients to illustrate helpful and effective communication situations. Other interdisciplinary palliative care team members' contact with patients and families were documented as: helpful (CH2, CH18, CH24, SW2, SW4, SW7, SW11), honest (CH4, CH18, CH24, SW22, SW16), and supportive (CH8, CH15, CH17, CH19, CH22, SW10, SW23).

Comparable behaviors from the chaplain interns and the social work interns involved providing support to the patient and family. The most similar behavior from both groups of interns involved encouraging communication between family members or with the patient (CH4, CH5, CH6, CH7, $\mathrm{CH} 8, \mathrm{CH} 10, \mathrm{CH} 11, \mathrm{CH} 14, \mathrm{CH} 15, \mathrm{CH} 16, \mathrm{CH} 19$, CH20, CH21, CH22, CH23, CH24, SW2, SW3, SW4, SW5, SW7, SW8, SW9, SW10, SW11, SW12, SW15, SW16, SW18, SW21, SW22). Chaplain interns and social work interns made a point to stop by to talk with patients and families. These visits often consisted of providing discipline-specific interventions (e.g., leading prayer for the chaplain interns or conducting family sessions for the social work interns). At times, the chaplain intern or social work intern sat with the patient and the family, providing support through physical presence.

Feelings of the chaplain interns and the social work interns translated into how they felt about interdisciplinary palliative care and the care they provided to the patient 
and the family. Both groups of interns described feeling strongly about the importance of comprehensive interdisciplinary palliative care for both patient and family $(\mathrm{CH} 1, \mathrm{CH} 2$, CH4, CH5, CH6, CH7, CH8, CH12, CH13, CH16, CH22, SW2, SW4, SW7, SW8, SW10, SW15, SW21, SW22). Both the chaplain interns and the social work interns also identified quality care from each discipline as essential (CH7, CH12, CH22, CH23, SW7, SW8, SW10, SW22).

In summary, similarities existed between the documented experiences, behaviors, and feelings of the chaplain interns and the social work interns from their interdisciplinary palliative care team placements. Comparable experiences for both groups were positive exchanges between interdisciplinary team members. Both groups described helpful communication as daily, constant, intentional, effective, and collaborative. Similar positive interdisciplinary palliative care team communication with patients and their families was documented as helpful, honest, and supportive.

The most similar behavior from both the chaplain intern group and the social work intern group involved encouraging communication between family members with the patient. Similar feelings from both sets of interns were solidly favorable on the importance of comprehensive interdisciplinary palliative care for both the patient and their family. Quality comprehensive care from each discipline within the interdisciplinary team was considered to be essential to many chaplain interns and social work interns.

\section{Conclusion}

This chapter described findings from the iCOPE Reflective Writing Assignment for this study. The emergent themes from the analysis of student intern reflective writings provide documentation of the experience of being on an interdisciplinary palliative care 
team for the interns. The themes of Learning Lessons, Disappointment, Being There, Dealing with Family Matters, and Making Hard Decisions were pertinent to both the chaplain interns' and the social work interns' experiences.

Two other emergent themes were discipline-specific, Intern as Existential Comforter for the chaplain interns and Intern as Psychosocial Resourcer for the social work interns. Each of these themes describes the behaviors that are unique to these disciplines, which were observed or performed by either the chaplain interns or the social work interns during their interdisciplinary team practicum. Finally, this chapter has reviewed the findings of the current study.

The next chapter provides a discussion of the study results. The limitations, conceptual model, and existential theoretical framework of the study will also be explored. Directions for future research and implications of the study conclude the chapter. 


\section{CHAPTER V: DISCUSSION}

This chapter examines the findings from the current study. As previously stated, all participants in this study are chaplain interns and social work interns who took part in a larger study (i.e., iCOPE study program funded by Robert Wood Johnson Foundation and awarded to the University of Louisville). The chaplain interns' and social work interns' documented experiences are in response to a series of established questions for the iCOPE study (see Appendix A) and serve as the primary data source for this dissertation study. Recall pre-existing data (e.g., documents and text) from chaplain interns and social work interns about their interdisciplinary palliative care team experience were used in the current dissertation study to explore the research questions.

The current dissertation study used chaplain interns' and social work interns' reflections collected during 2012-2015 to explore the research questions. These written reflections were responses to 10 questions (see Appendix A) regarding their experiences during their clinical rotation in the iCOPE study. This single reflective written assignment was required of all students in the iCOPE study. The interns submitted their reflections to the iCOPE Program Coordinator at the end of each semester for the duration of the study. Importantly, the language data were used because it specifically related to participants' unique clinical experiences working on an interdisciplinary palliative care team, which was the focus of the current study.

The final chapter of this dissertation reviews the research problem and describes the qualitative methods used in the study. It contains the discussion of the findings, comparison of the findings to the literature, including conceptual framework, 
conclusions, recommendations, and directions for future research. Implications for diverse providers, including the counseling profession, will also be offered.

\section{Discussion of Study Results}

Themes from the current study are similar to the literature which supports it. The themes from this study are also shaped by the interdisciplinary palliative care team environment. Interdisciplinary team member role knowledge and role flexibility are important aspects of an effective interdisciplinary team. Communication is the essential feature of team collaboration; however, communication breakdowns still occurred. Other factors which emerged from this study will also be discussed.

\section{Interdisciplinary Palliative Care Teams}

In the current study, the interdisciplinary palliative care teams that served as clinical placements for the chaplain interns and social work interns provided them with helpful experiences for learning how to provide care for the patient and the family. Learning for the interns included team collaboration about the patient, team creation of a plan of care for the patient, and team work for patient goals. Arguably, the best interdisciplinary palliative care team experience for an intern is placement on a highly functioning interdisciplinary care team.

Research suggested exposure to disciplines who work together as helpful for inter-professional education. Youngwerth and Twaddle (2011) supported the integration of interdisciplinary teamwork into professional healthcare education and find it is vital for high quality palliative care. Both groups of interns completed their interdisciplinary education and clinical palliative care placement as part of an interdisciplinary palliative 
care team, which are considered essential for future patient-centered practice (Head et al., 2012).

Findings from the current study suggest the chaplain interns and social work interns seem to have been exposed to highly functioning interdisciplinary palliative care teams. Teams who were knowledgeable and respectful about all team disciplines and roles and possessed excellent collaboration and communication skills align with NCP Standards (2013). Interdisciplinary palliative care teams who were high performing will be in the best position to give the interns experiences so they learn how to become effective team members. The vast majority of all interns appear to have been placed with teams that were effective and beneficial to their patients and families.

International and national agencies recommend inter-professional education (i.e., education that involves educators and learners from two or more health professions) to prepare professionals to provide palliative care. The mandate from the WHO considers inter-professional education as necessary for a collaborative practice-ready health workforce, which is a worker who has learned how to work on an interdisciplinary team and is competent to do so (WHO, 2010). Both groups of interns reported training and interdisciplinary team experiences; these prepared them to be capable interdisciplinary team workers.

The IOM (2014) stated in its most recent report that special practice in hospice and palliative care in the education of health professionals has greatly improved; however, problems still remain. Often-reported challenges include the following: a) insufficient attention to medical and nursing school curricula; b) educational silos exist which inhibit the development of inter-professional teams; and c) deficits in preparing 
physicians with appropriate communication abilities. Attention to hospice and palliative care education has progressed as evidenced by the creation of an interdisciplinary palliative care education program (i.e., iCOPE) for different groups of healthcare workers (e.g., medicine, nursing, chaplaincy, and social work) at the University of Louisville.

In order for an interdisciplinary palliative care team to function at an optimal level, the team must possess outstanding training, excellent communication, description of team tasks, and responsibilities for each team member (Vissers et al., 2012). In the current study, both groups of interns described collaboration with all domains as they attended formal interdisciplinary team meetings or observed informal instances of communication between disciplines. Often nurses interacted with chaplains or social workers and updated them on patient needs. The chaplain interns and social work interns also reported ways they informed nursing staff of changes in the patient's condition.

It is important for the represented disciplines to contribute and collaborate in interdisciplinary team meetings. Research demonstrated a gap often exists in non-medical (i.e., social workers, chaplains) contributions and may explain why collaboration was not present in $30 \%$ of patient care discussions (Wittentberg-Lyles et al., 2010). Interestingly, of the interns who were present for patient care discussions, none reported a disparity in non-medical reports. In fact, the chaplain interns often assessed and contributed the spiritual assessment to the plan of care for their patient. It is crucial that the patient's plan of care contain input from all disciplinary areas, because patient care may suffer without representation from each discipline.

Several social work interns described how much their patients liked to participate in their plan of care meeting with the interdisciplinary care team. The social work interns 
observed the social worker present psychosocial assessment for consideration in the patient's plan of care. Comprehensive patient care contains information from each of the disciplines for the goals of care. Collaboration occurs when all team members' knowledge is appreciated and considered within the treatment goals (Fewster-Thuente \& Velsor-Friedrich, 2008).

\section{Interdisciplinary Team Member Roles}

Members of interdisciplinary care teams have flexible roles in order to care for patients and families. All members of the team are expected to be resourceful as they meet the needs of the patient and family (Bronstein, 2003). However, this very aspect of interdisciplinary teamwork has been problematic for role definition. Role flexibility and ambiguity have been reported as challenging for psychosocial team members in the literature (Junger et al., 2007; Nancarrow et al., 2013; Wittenberg-Lyles et al., 2008).

In the current study, no difficulties with role flexibility or ambiguity were reported from either group of interns, which could mean the interns were very clear on the limits of their roles. Specifically, the chaplains' professional role on the team was found to be unclear on the interdisciplinary team because other team members did not understand it (Wittenberg-Lyles et al., 2008). Social workers are striving to be valid and recognized members of the interdisciplinary palliative care team; however, within-team tension may result from frustration with unclear role boundaries from the psychosocial domain (O’Connor \& Fisher, 2011). As described in the literature, the roles for chaplains and social workers on an interdisciplinary team appear to be unclear.

Psychosocial care is shared by the interdisciplinary care team; however, research has shown this is a contested area of patient care that social workers, chaplains, palliative 
care nurses, counselors, and psychologists want to claim (O'Connor et al., 2006). The documented reflections of the chaplain interns and social work interns did not verify any challenges regarding provision of psychosocial care for patients or their families. Meier and Beresford (2008) determined the historical power differences between physicians and psychosocial-spiritual professionals and standard hospital cultures can cause problems in leadership and therefore, affect interdisciplinary team work. Once again, no issues were reported in power between physicians and group of interns.

The documented chaplain interns' roles were very similar to those found in the literature. They took primary responsibility for spiritual care for the patient, the family, friends, and colleagues (Harper \& Rudnick, 2010). The chaplain interns functioned as clergy liaison to outside ministers and participated in funeral preparations (WittenbergLyles et al., 2008). One chaplain intern asked to contact a patient's minister for family spiritual support and another arranged the funeral for a patient because doing so was causing more stress in the family of the patient. Chaplain duties differed from other team members because they go beyond routine patient visits and regular interdisciplinary palliative care team meetings.

Chaplain interns were present with the patient, made spiritual assessments, and gave spiritual care planning (Millspaugh, 2005). Chaplain interns viewed themselves as those who improved family and team communication (Fitchett et al., 2011). These aspects of spiritual support were documented in the reflective writings when the chaplain interns gave spiritual assessments in formal team meetings, and also within informal spiritual communication with other members of the team, with patients and their families. 
Often chaplain interns described providing presence to the patient, and in doing so, served as a witness to suffering and a mirror of unconditional love to the patient (Puchalski, Lunsford, Harris, \& Miller, 2006). Additionally, chaplain interns observed and supported the families whose loved ones received compassionate extubation. In cases such as these, they gave help to families who make difficult decisions pertaining to endof-life (Flannelly et al., 2012).

The chaplain also acted informally as the team's conflict manager and daily support for the team members (Wittenberg-Lyles et al., 2008). The other disciplines (i.e., medicine, nursing, social work) are not expected to fulfill such informal roles for the interdisciplinary team. The role of interdisciplinary team conflict manager was documented by a chaplain intern when there was a disagreement between physicians that caused tension within the team (Wittenberg-Lyles et al., 2008).

Role flexibility occurred between the chaplain interns and the social worker, not the social work intern, on the interdisciplinary palliative care team. Bornstein's (2003) model of two-part optimal interdisciplinary collaboration supports role flexibility because effective collaboration demands it. However, there was no evidence of role confusion or role ambiguity in cases between the chaplain interns and the social workers or social work interns when patient spiritual support was delivered. The documented reflections of the chaplain interns did not reveal any within-team tension with the social workers or social work interns who provided spiritual care.

Two themes that are related to the roles of the chaplain intern are: Learning Lessons: Being a Chaplain and Intern as Existential Comforter. Both themes align with 
the behaviors and actions of the chaplain role. The first theme, Learning Lessons: Being a Chaplain, described the learning gained from working in the chaplain role on the interdisciplinary team. The theme of Intern as Existential Comforter contains the unique contributions chaplain interns make on the interdisciplinary team and their interventions for the patient and family. Chaplain interns reported similar feelings with the social work interns about interdisciplinary palliative care teams. They reported interdisciplinary care is important and care from each discipline is essential.

Social workers are an integral and appropriate part of providing good palliative care by delivering assessment and intervention within the social and psychological wellbeing of the patient, family, friends, including caregivers, before and after death (Monroe, 2010). Social workers modeled appropriate and helpful behaviors for the social work interns during their interdisciplinary palliative care team placements. Palliative care social workers were observed by social work interns as engaged in team discussions with the patient and family about disease progression, goals of care, and initiated dialogue of and completed paperwork regarding advance directives. Social workers were reported to educate the family on anticipated caregiving needs for the patient and other dependents, as mentioned in the literature (Blacker \& Deveau, 2010).

Social workers were aware of the "person-in-environment" (Bornstein, 2003, p. 298) and provided contextual understanding of the patient's complex symptoms for the plan of care. Multiple families were reported to have challenging financial issues and other pressing needs (e.g., family discord, long-standing traumas, and caregiver burden), which impacted social worker and social work intern ability to provide solutions for the patient, which was supported in the literature (Klarare et al., 2013). Social workers were 
documented as integrating the biopsychosocial aspects of the patient and the family into the comprehensive goals of care, which is part of their role on the team (NASW, 2004).

Social workers and their interns had a vital presence on the interdisciplinary team. As documented by the social work interns, they cared for suicidal patients, facilitated family sessions, and provided support for several families who needed help to talk with young children about death. The palliative care social worker facilitated referrals to hospice providers, as observed by the social work interns. Grief and bereavement followup were mentioned as part of the social worker responsibilities by social work interns, but were not provided by them at their placements. Ethics consultations are also the domain of the social worker (Blacker \& Deveau, 2010) and one social work case was referred for an ethics committee decision.

Role flexibility in the spiritual realm was documented by a social work intern. One of the social work interns reported providing spiritual presence and active listening to a patient; however, she felt uncomfortable because she did not know how to help. Wesley et al. (2004) found social workers are reluctant to intervene because they do not believe they possess the knowledge to provide spiritual care. The patient, in this instance, had been offered chaplaincy services; however, he enjoyed talking to all team members about his faith. The patient's spiritual need to discuss his religious convictions was met by a social work intern. Spiritual requests can be met by nurses and social workers, not only by chaplains, according to patients (Edwards et al., 2010). This documented reflection was a very clear example of role flexibility that met the patient's need at the moment he required it. 
Two themes also correspond with the role of social worker on an interdisciplinary team. These were Learning Lessons: Being a Social Worker and Intern as Psychosocial Resourcer. Social work interns observed their mentors during their interdisciplinary team placement. They learned from them how to perform and respond as social worker on the team. The theme of Intern as Psychosocial Resourcer aligns with the distinctive contributions made by the social worker on the team. Social work interns described a similar set of feelings with the chaplain interns regarding interdisciplinary palliative care teams. They reported interdisciplinary care is important and care from each discipline is essential.

\section{Communication}

Not surprisingly, communication was found to be very important within the documented reflections of the chaplain interns and social work interns. Both groups of interns were asked to describe communication with the team, among each other, the patient, the family, and other providers in their reflective writing assignment. The themes that emerged from the reflected writing assignments were all related to communication.

To assert that interdisciplinary team communication is important is an understatement. The literature described communication as the essential factor for interdisciplinary team efficacy (Bronstein, 2003; Sargeant et al., 2008). However, effective team communication is not easy to create. Substandard communication and team communication are constant challenges (Hartrick-Doane et al., 2012) and effective interdisciplinary team functioning does not happen without effort and awareness (O'Connor \& Fisher, 2011). Without question, the effective interdisciplinary care team 
must communicate with the team during formal plan of care meetings, between team members in informal ways, and with the patient and family.

Effective communication on the team precedes team collaboration; factors that promote collaboration are respect, sharing, and trust (Henneman et al., 1995). Communication and collaboration among the team members are vital for providing quality palliative care. Cooperation among team members is an important foundation for collaboration and communication; this pair of concepts are reciprocal and dynamic, with each enhancing the other (Feudtner, 2007).

As previously mentioned, communication is crucial for the effective interdisciplinary care team. It is the primary feature of interdisciplinary palliative care team collaboration (Bokhour, 2006; Goldsmith et al., 2010; Junger et al., 2007; Sargeant et al., 2008). Thus, as expected, in the current study the chaplain interns and social work interns described their interdisciplinary care team communication in the following ways: demonstrated respect to each other, were aware of each member's responsibilities, worked together for patient and family, were warm and receptive to family, and saw team members treat each other amicably. Communication within the team was also positive, defined as good and proactive. Both groups of interns remarked on helpful and up-to-date communication within the team, which is a hallmark of a high functioning interdisciplinary team (Bokhour, 2006).

Themes from data analysis of both intern groups' reflective writings revealed multiple instances of communication related to team communication. Learning Lessons: From the Team, Being There: With the Team, Dealing with Family Matters, and Making Hard Decisions were themes that involved communication. 
Learning Lessons: From the Team and Being There: With the Team were themes that encompassed knowledge acquired for teamwork from the interdisciplinary team. Learning how to communicate with patients and their families was another area where the interns acquired important contact. Dealing with Family Matters and Making Hard Decisions were themes that aligned with the communication dynamic from the team to patient and family. The theme of Dealing with Family Matters concerned communication, either positive or negative, received from the patient's family. Making Hard Decisions was another communication theme involved in treatment choice for the patient. The family made the care decision after clear communication had been provided by the interdisciplinary team.

Communication with the patient and family is also vitally important. The theme of Being There: For the Patient and Family is described as experience gained by the interns during their placement primarily from interaction with the patient and family. Effective communication with the patient and family was evidenced by the chaplain intern and social work intern documented reflective writings primarily as honest and supportive. Both intern groups' explained their similar behavior to be focused on encouraging communication, usually with the patient. As part of the reflective writing assignment, the interns described how patient and family needs were met during their placements; if their needs were not met, they gave constructive feedback for ways the needs could be met.

Similar communication knowledge themes were documented by both groups of interns. The chaplain interns and the social work interns provided comparable experiences, behaviors, and feelings regarding overall communication. Both groups 
described effective team communication as daily, constant, intentional, effective, excellent, collaborative, and helpful.

The findings from this study were positive about interdisciplinary team communication processes as reported from the interns' reflective writing assignments. Both groups of interns were knowledgeable about interdisciplinary care team functioning and comprehensive interdisciplinary care as described in their reflective writing assignments. This reflects education about the aspects of useful interdisciplinary palliative care teams. Unfortunately, high-quality interdisciplinary palliative care is not always available. Research has found patient-care is often not as accessible, coordinated, patient-centered, or evidence-based as it could be (Ferrell et al., 2014).

\section{Communication Breakdowns}

Communication is of critical importance to the interdisciplinary palliative care team and the patient; however, breakdowns in communication do occur. Patients who are at the end-of-life, and their families, are often overwhelmed and vulnerable. The interdisciplinary care team is obligated to respond to the patient and family when a breakdown in communication happens. Communication breakdowns negatively affect interdisciplinary team operation (Goldsmith et al., 2010; Mazor et al., 2012).

Mazor et al. (2012) found communication breakdowns caused emotional or psychological consequences to patients. Similar to Mazor and colleagues, the study participants described numerous breakdowns in communication. The chaplain intern group reported instances of three types of communication breakdown: a) physician to team, b) team to family, and c) patient to physician. In the first case, the patient's primary oncologist stated the interdisciplinary palliative care team had given up on the patient, 
which diminished trust the family placed in the team. In another example, discussion about scheduled labs, then cancelled labs, was confusing for the family and was problematic for the dying patient's emotional comfort.

In the last communication breakdown example reported by a chaplain intern, the patient asked for a palliative care consultation that was supported by her interdisciplinary palliative care team. The physician cancelled the palliative care consultation and the patient received medication for depression. As is often the case, a breakdown in communication may have caused unnecessary worry and emotional distress for the patient and family.

The social work interns also recorded several instances when communication collapsed. There were four types of communication breakdowns: a) between two medical services, b) physician to team, c) team to patient, and d) patient to physician. Two communication breakdowns between services were documented by the social work interns. One collapse was between the respiratory service who extubated a patient without notifying the interdisciplinary palliative care team when this would occur, and another collapse between services happened when a patient received less than optimal care during the night shift. In this case, the patient's son lost trust in the staff's ability to provide for his father.

Also, a social work intern observed the lack of communication between the physician and the interdisciplinary care team which may have limited treatment choices for the patient. Another perceived communication failure was recorded as a collapse within the interdisciplinary palliative care team to the patient. In this case, neither the patient nor the family had been informed that the patient's death was imminent. Finally, 
another patient was refused a hospice evaluation and was referred for a psychiatric evaluation by her physician.

Breakdowns in communication were observed by both groups of interns. A lack of communication between professionals or the family, failure to communicate between services, and the apparent reversal of hospice referrals which became psychiatric referrals instead, caused patients and families to experience emotional distress and loss of trust at a very fragile time. Communication breakdowns were documented by both groups of interns and also supported in the literature. In providing patient-centered care more consideration should be given to improved communication with patients that may prevent problems in their care (Mazor et al., 2012).

Although patients are often the interdisciplinary team's center of attention, a qualitative study found family members and caregivers were often the primary focus of care for the interdisciplinary team (Goldsmith et al., 2010). The communication failures in the current study may be considered to be opportunities for the entire team to improve service to patients and communication with each other. Membership on the interdisciplinary palliative care team helped insure that patient and family needs were met, because the needs at the end-of-life are too vast for any one individual (Goldsmith et al., 2010).

The theme associated with communication breakdowns was Disappointment. Overall, eight interns observed a communication breakdown. Of the documented communication breakdowns, several were with physicians. Two physicians decided to change a palliative care consultation that was supported by the patient and the interdisciplinary care team, which is troublesome, but not unexpected. Problems with 
physician communication were reported by the interns in this study and also by the IOM (2014) in its latest report. At a time when the patient and family are overwhelmed with end-of-life concerns, failures in communication cause more difficulties for everyone.

Barriers and challenges that negatively affect interdisciplinary palliative care team performance often reported in the literature were: the lack of a common language between team disciplines (Gachoud et al., 2012; Mellor et al., 2002), limited input from nonmedical team members and biomedical dominance (Wittenberg-Lyles, Oliver, Demiris, \& Regehr, 2009; Wittenberg-Lyles et al., 2010), existence of a traditional biomedical hierarchy (Abramson \& Mizrahi, 2003; Hartrick-Doane et al., 2012), and role flexibility and ambiguity (Bronstein, 2003; Junger et al., 2007; Klarare et al., 2013; Nancarrow et al., 2013; Wittenberg-Lyles et al., 2008).

Interestingly, there was no evidence from the interns' reflective writing assignment of barriers or challenges caused by the lack of a common language between the team disciplines (Gachoud et al., 2012; Mellor et al., 2002). No interns reported instances of observed medical dominance, which can be responsible for team imbalance (Klarare et al., 2013). Actually, several interns documented that their teams requested more medical input, especially from the physician, for decision making.

In order to provide comprehensive patient care and avoid communication breakdowns on the team, each of the disciplines provides an assessment of the patient, which frames the plan of care. Previous studies of effective interdisciplinary team work have shown support for the inclusion of assessments and input from all members of the interdisciplinary team (Bokhour, 2006; Klarare et al., 2013, Vissers et al., 2012; Youngwerth \& Twaddle, 2011). However, some studies have shown chaplains and social 
workers are left out of team discussions due to the predominance of biomedical information (Wittenberg-Lyles, Oliver, Demiris, \& Regehr, 2009; Wittenberg-Lyles et al., 2010). Also, the existence of traditional biomedical hierarchy is often a barrier to team collaboration and in most medical teams the physician is the leader; however, this is not so in the democratic environment of interdisciplinary palliative care teams (Abramson \& Mizrahi, 2003; O'Connor et al., 2006). Surprisingly, none of the interns in this study substantiated the existence of any of the above barriers to interdisciplinary team collaboration.

Communication breakdowns may occur when team members do not possess all patient information. The structure of nurse as primary reporter in the interdisciplinary meetings has been found to be a barrier that inhibits other disciplines from sharing information for discussion about all aspects of the patient's care (Wittenberg-Lyles et al., 2009). Often, non-medical disciplines are left out of interdisciplinary discussions; however, there was no indication from the intern groups that the nurses who lead team meetings inhibited the sharing of patient information from the chaplain interns or the social work interns. In fact, one chaplain intern described a formal team meeting where the nurse assumed the leadership role and asked for the assessment from each discipline as well as other information to inform the patient's plan of care.

There was also no indication from either intern group that nurses who led team meetings inhibited sharing of information, which could have impacted team communication breakdowns. The historic power and responsibility imbalance between medicine and psychosocial and spiritual team members (Meier \& Beresford, 2008) was 
not demonstrated in any of the intern reflective writings. I did not detect evidence of biomedical hierarchy presented by the interns.

\section{Other Factors}

Other contextual factors which were part of the interdisciplinary palliative care team experience for the interns were: an atmosphere death and dying and spirituality on the interdisciplinary team. The literature reported interdisciplinary team members must have the ability to cope with death and dying patients, their personal bereavement, and possess the ability to support grieving family members (Junger et al, 2007).

Interdisciplinary teams were also found to have a spiritual atmosphere (Clark et al., 2007).

Placement on an interdisciplinary palliative care team presented actual contact with dying patients and their families. Several social work interns described feeling overwhelmed with the atmosphere of death; however, none of them reported a loss of interest in the palliative care field as a result of their placement. Not unexpectedly, the chaplain interns did not report personal difficulties dealing with the dying or their families.

A high prevalence of spirituality among interdisciplinary teams was found in an empirical study by Clark et al. (2007). Surprisingly, in the current study, spirituality as part of the culture of interdisciplinary palliative care teams was not explicitly described by the interns. However, several social work interns wrote about revered topics (e.g., impending death or making meaning with one's remaining time) as they participated on the team. 
Some social work interns wrote about their observations or experiences using inspirational language, which indicated describing end-of-life work in a transcendent way. One expected chaplain interns to use spiritual language to frame their reflections, which some did. A feeling of palpable spirituality on the unit was not described by the interns as it was by the participants in the Clark et al. (2007) study.

Overall, the interdisciplinary palliative care placement experience was reported to be a positive learning experience by both groups of interns. Many interns, especially the social work interns, wrote they would consider palliative care or oncology as a future work placement. For the others, the placement experience reaffirmed their desire to work in the palliative care field.

In the current study, the interdisciplinary palliative care team placement experiences as described by the chaplain interns and social work interns were filled with beneficial learning. Not only did the interns report gaining knowledge from their placements, surprisingly, virtually none of the interdisciplinary care teams were reported by the interns to have any of the expected barriers and challenges (e.g., lack of common language between disciplines, existence of traditional biomedical hierarchy, lack of input from non-medical disciplines, biomedical dominance, and role ambiguity) found in the literature. Communication breakdowns occurred, but these failures were primarily between services and involved physicians, rarely were there difficulties within the team.

Psychosocial care provision was not contested by the members of the team, nor were there any problems with role sharing. The conceptual framework also supports the efficacy of the reported interdisciplinary palliative care teams. Interdisciplinary care education may have evolved to the point that it is now prepared to hold its own place in 
the medical world. It could be that interdisciplinary and inter-professional education and practicum are now established locally and have transformed palliative care in this area. Evidence from the documented experiences of chaplain interns and social work interns strongly support a learning environment for chaplain interns and social work interns of highly functioning interdisciplinary palliative care teams.

It could be a number of clinicians, of any discipline, have interdisciplinary training and are in supervisory positions where they train or influence other clinicians through high performing interdisciplinary care teams. Perhaps interdisciplinary education has created enough healthcare professionals who understand and work with others in an interdisciplinary manner. They see the benefits of comprehensive interdisciplinary palliative care provided by teams and received by patients and families at the end-of-life and are willing to keep the program moving forward to be at the forefront in palliative innovation.

It could be that the results from the SUPPORT study (Connors et al., 1995) from 20 years ago no longer describe the bleak state of affairs for those at the end-of-life. Effective interdisciplinary palliative care teams now exist. A knowledgeable, interprofessionally trained workforce of different disciplines is ready to provide comprehensive physical, emotional, psychosocial, and spiritual compassionate patientcentered care to the dying and their families, although much more research is needed.

\section{Relevance of Bronstein's Conceptual Model}

In addition to the examination of the planned research questions, a secondary aim of the current study was to explore Bronstein's conceptual model of interdisciplinary communication. Bronstein's model of two-part optimal interdisciplinary collaboration 
(2003) is an existing conceptual framework of interdisciplinary communication applied in the current study. This theoretical model is used to examine aspects of teamwork reported by the chaplain interns and social work interns as documented in their reflected writing assignment after their interdisciplinary care team placement. Bronstein (2003) stated five inter-professional processes among professionals from different disciplines should denote activities, c) flexibility, d) collective ownership of goals, and e) reflection on process.

Interdependence is the reliance on other team members where all are dependent on the other to accomplish goals. In order for interdependence to occur on the interdisciplinary team, each professional must have a clear understanding of their own role and the roles of the other professionals, and use them appropriately (Bronstein, 2003). Characteristics of interdependence are formal and informal time spent together, oral and written communication within the team, and respect for colleagues' professional opinions and contributions.

Examples of interdependence were discovered within the interns' reflective writings. One chaplain intern recalled how the interdisciplinary care team worked together to support a patient and her family as she unexpectedly and rapidly declined while in the hospital. The patient's goals of care were to be comfortable and pain-free; however, her husband wanted her to receive heroic measures to keep her alive. The chaplain intern and the palliative care physician suggested use of heroic measures (e.g., CPR and/or chest compressions) would be painful for her husband to witness and would be contrary to her goals of care. Another instance of interdependence within the interdisciplinary care team was documented by a social work intern as she reflected upon her palliative care experience with a military veteran. The intern observed the activities 
and behaviors of interdependence, which were team members treated each other with respect, each member presented their individual assessment into the plan of care, and participated in the team meeting.

Newly created professional activities comprise the second component of Bronstein's (2003) model of optimal interdisciplinary collaboration. Newly created professional activities are defined as collaborative acts in which professionals achieve more together than they could independently. Comprehensive care provided by an interdisciplinary care team that created a plan of care for each patient, is an example of what may be achieved when diverse disciplines work together.

Instances of newly created professional activities within an interdisciplinary palliative care team were defined in the reflections of a chaplain intern and a social work intern. The chaplain intern described how time was given to the patient, which created an atmosphere for him to make a difficult decision. The patient identified a goal, with support from the chaplain intern, which would help him determine when to begin palliative care. The social work intern observed the interdisciplinary team set up a plan of care which worked well for the patient and his family. The interdisciplinary team consolidated the patient's appointments which helped the family and the patient see professionals from multiple disciplines so many of their needs were addressed in one session. This action saved effort for the family and stress on the patient.

Flexibility within Bronstein's (2003) model for interdisciplinary collaboration is defined as deliberate role-blurring, when professionals respond creatively to what is needed. Flexibility was demonstrated multiple times as the interns wrote about doing jobs typically expected of the other interns. In one instance, the chaplain intern observed the 
social worker talk to the patient about a Living Will, which is usually the chaplain's responsibility at that hospital. In a different case of flexibility, the patient initiated a talk about faith with the social work intern on his interdisciplinary team. Although the social work intern felt uncomfortable in this role, she demonstrated flexibility within her role in response to his desire to talk about his spirituality. The social work intern supported the patient as she listened to him at exactly the moment he needed it.

Collective ownership of goals is another component of Bronstein's model (2003). This refers to the team's shared responsibility in the entire process of developing, designing, and achieving goals. To engage in collective ownership of goals, all team members take responsibility for the success, the failure, and support constructive disputes and discussion among the team. In this case, the chaplain intern described the interdisciplinary team as large (i.e., physicians, nurses, pharmacist, social worker, and chaplain) and wrote the team provided support to the family. The team maintained the plan of care for this patient and the chaplain intern remarked nearly everyone on the team worked together at the same time.

An instance from a social work intern illustrated the collective ownership of goals component described by Bronstein (2003). The interdisciplinary palliative care team shared the responsibility of this patient's care with her daughter, the patient's legal guardian, who lived out-of-state, and the patient's caregiver, who was a close family friend. Multiple conference calls between the team members (e.g., social worker, doctor, and nurse) caregiver, and daughter were made in order to communicate about placement options and the effects of medication. The collective effort of the team achieved the 
patient's goals (e.g., pain management) and solved the difficulties with the out-of-state daughter.

The last component of the first portion of the Bronstein model of interdisciplinary collaboration (2003) is reflection on process. Reflection on process refers to team members' attention to their process of working together. The chaplain interns and social work interns review their interdisciplinary palliative care team experience by completing a reflective writing assignment and group discussion. The element of reflection on process within Bronstein's (2003) model was not disclosed to have occurred by the teams in the field.

Bronstein's (2003) second part of optimal model of interdisciplinary collaboration includes other influences on interdisciplinary collaboration. These are: professional role, structural characteristics, personal characteristics, and history of collaboration. When these influences exist on an interdisciplinary team, interdisciplinary efforts are supported; when they are absent, a barrier exists.

The influences within the second part of Bronstein's model (2003) are defined as: professional role, which is a strong sense of ones' professional role as having similar values, ethics, and perspective to other team members. One's professional role and sense of being a team member must be balanced. Another influence is structural characteristics, which are relevant to interdisciplinary collaboration. These are: manageable caseload, supportive agency culture, administrative support, professional autonomy, and time and space for collaboration to occur. It is unable to be determined if the influences of professional role and structural characteristics as defined by Bronstein (2003) existed from the chaplain intern and the social work intern written reflections. 
The following two influences, personal characteristics and history of collaboration, are supported by available evidence from the interns' experiences. Personal characteristics consist of trust, respect, understanding, and informal communication between collaborators. Respect between collaborators and informal communicating were mentioned in the documented interdisciplinary team communications and experiences by both the chaplain interns and the social work interns.

A history of collaboration refers to earlier experiences from interdisciplinary settings with colleagues. Bornstein (2003) suggested positive experiences with interdisciplinary collaboration are shown to be linked with current levels of successful collaboration. The interdisciplinary palliative care team experience was a clinical learning opportunity and now having had this learning, the interns may perform at a higher level in future interdisciplinary team situations.

The conceptual framework of Bronstein's model of interdisciplinary collaboration (2003) was demonstrated to undergird interdisciplinary collaboration as evidenced by the iCOPE Reflective Writing Assignment by the chaplain interns and social work interns. Nearly all aspects of Bronstein's model (2003) of optimal interdisciplinary collaboration were verified by their documented experiences. The interns appeared to be well prepared for clinical placement and the interdisciplinary palliative care teams also seemed to perform at a high-level because nearly every aspect of the conceptual model was verified through the interns' reflective writing assignments.

\section{Existential Theory and Palliative Care}

An existential approach for the interdisciplinary palliative care team was evidenced by the queries posed by patients and families, which were often concentrated 
on what was precious, unique, or problematic in their lives (Tomer \& Eliason, 2008). Many families faced difficult treatment choices, and within the theme of Making Hard Decisions, the interns described conversations about their dying loved ones. In some instances, the interns themselves wrote about treasured aspects of their patients.

The interdisciplinary palliative care team, and the patient and family, are exposed to a death boundary encounter (May \& Yalom, 1995). This urgent experience may cause a powerful change in perspective for the patient and the family. Several patients explained their spirituality had become greater and as a result of their diagnosis, they had changed how they wanted to live the remainder of their lives. The threat of death may transform how one sees themselves as well as how they perceive death (Tomer \& Eliason, 2008).

According to the Tomer and Eliason (2008) existential model of death anxiety, three types of regret predict how one views death, as either meaningful or meaningless. The types of regret are defined as: Past, which contains emotion related to the past; Future, which means action in the future is impossible, and one's View of Death, whether it makes sense or is meaningless. This social work intern illustrated a patient who could have death anxiety. He had many longstanding family difficulties which included many traumas throughout his life. The patient had lost his young son and had been estranged from his daughter for nearly twenty years. He may suffer from a higher level of death anxiety because he holds unresolved past regret and future regret, which may make his death appear to have no meaning. 
The interdisciplinary palliative care team also encounter death as a boundary situation. They lose patients daily and have the opportunity to witness existential explorations of transcendent matters by the patient and the family (Breitbart et al., 2004). Awareness of existential issues and difficulties with the life regrets of the dying may inform the team and help them provide suitable care of the patient and the family. According to Sinclair et al., (2006) one of the most important roles of the interdisciplinary palliative care team in providing spiritual care is to honor and listen to the suffering of the dying.

\section{Limitations}

The current study had several limitations. The first limitation in this study was the lack of generalizability from qualitative research. Findings cannot be generalized; however, they can be generalized most confidently to settings and populations that are most similar to this research (Patton, 2015). Generalizability was not an aim of this study; however, the hypotheses generated by qualitative research may be used for further studies.

Second, this study was limited to the pre-existing data derived from the larger iCOPE study, demographic data collected, and the 10 questions asked of the study participants. Race, gender, and age were demographic limitations within the current study. Toward this end, the race of the iCOPE participants was not collected. Collection of this information would be useful in better understanding the racial and ethnic diversity of the interdisciplinary teams. Gender may have had an effect on the findings, especially given the overwhelmingly high number of females within the social work intern sample. Only two males were in this sample of social work interns $(\mathrm{N}=23)$. The chaplain interns 
$(\mathrm{N}=24)$ were evenly split (males: $\mathrm{N}=12$ and females: $\mathrm{N}=12$ ). Also, the mean age between the two intern groups may have influenced the findings, as the chaplain interns had a higher mean age $(X=41)$ than the social work interns $(X=29)$.

Third, there was significant discrepancy between the intern groups and the amount of direct care they provided. The chaplain interns provided direct interventions and patient care, while the social work interns observed their mentors determine interventions and deliver direct care to the patient and family. The difference in direct care provision caused a difference between the groups.

Fourth, the lack of pediatric patients within the patient sample was another study limitation. All palliative care patients with whom the chaplain interns and social work interns worked were adults. Children comprise a portion of the dying and it was unexpected that none of the interns encountered a pediatric palliative care case. Findings may have been impacted by the adult only cases.

Finally, the assignment of social work interns to Hosparus (i.e., the local hospice agency), added a different dimension to the placements of the social work interns. The social work interns experience palliative care delivered by an interdisciplinary care team which operated under different guidelines than those in area hospitals. The social work interns who had a clinical palliative care placement with Hosparus had a somewhat different experience (e.g., home visits or in-patient hospice units) than the other interns who were placed on interdisciplinary palliative care teams. The social work interns who participated in Hosparus home visits had a distinctive palliative care environment from those who had in-patient hospital placements. Findings from the social work intern group 
may have been influenced by their slightly altered placement when viewed alongside the chaplain interns who were exclusively placed in Louisville area hospitals.

\section{Directions for Future Research}

Qualitative research may be used to generate hypotheses and future studies are needed in the field of palliative care provision. Prospective empirical research on interdisciplinary palliative care teams could use a quantitative or mixed methods approach. Quantitative research into interdisciplinary palliative care teams, made up of the traditional four discipline composition, not including hospice care, is limited (Higginson et al., 2002). Research of interprofessional collaboration or palliation have been the subject of systematic reviews (Zimmerman et al., 2008; Zwarenstein et al., 2009) but more research is needed.

Often delivery of palliative care is not by an interdisciplinary team, or if it is, team composition is not consistently represented by the traditional four domains within interdisciplinary palliative care (e.g., medicine, nursing, chaplaincy, social work). A systematic review of palliative care concluded evidence of benefit from palliative care was scarce and limited by methodological shortcomings (Zimmerman et al., 2009). Future studies to examine effective interventions for improved quality of life and care satisfaction from highly functioning non-hospice inpatient interdisciplinary palliative care teams could be the foundation of this research. The interdisciplinary palliative care teams that served as placements for the interns in the current study appear to be performing at a high level. Research may now build upon the strong non-hospice inpatient interdisciplinary palliative care team foundation which presently exists. 
Palliative care programs will be needed for the large group of elderly patients who will comprise nearly 20 percent of the total U. S. population by 2030. Many within this group of elders will have chronic disease which will make them ineligible for hospice care. They will require high quality end-of-life care programs and hospitals would benefit from knowing how to supply effective interdisciplinary palliative care team programs.

Highly functioning non-hospice inpatient interdisciplinary palliative care teams already exist. New evidence-based information may come from the study of them for use in future programs. Palliative care programs, staffed by traditional interdisciplinary palliative care teams are one way hospitals may grow to meet the future demand of elderly patients.

\section{Implications for Future Interdisciplinary Teams and Training}

Basic education for clinical professionals should include the knowledge, skills, and attitudes required for effective interdisciplinary team participation and this education should be supported within the practice setting (Ponte et al., 2010). According to the documented reflective writings, the interns appeared to be well prepared for their interdisciplinary palliative care team placement. Although one qualitative study cannot provide a sound basis for the determination of palliative care learning, this study would suggest the current foundation of didactic and clinical practica used produced positive interdisciplinary team experiences for both chaplain interns and social work interns.

Interestingly, difficulties for psychosocial (e.g., non-medical) interdisciplinary palliative care team members can be challenging and they struggle to find their place on the team; however, this was not evidenced in the current study (O'Connor \& Fisher, 2011). Highly functioning and well-communicating interdisciplinary palliative care 
teams are not easy to create. The interns in the current study were exposed to effective interdisciplinary teams and gained interprofessional education, which is crucial for students who are preparing to work in today's healthcare settings (Head et al., 2014).

The evolution of an interdisciplinary team takes time as the professionals grow into their roles and learn how to work together; however, the process can be "conscious and planned, or unconscious and accidental” (Remke \& Schermer, 2012, p. 291). The process of preparing future professionals for interdisciplinary team work has been a conscious endeavor, carefully planned over time, throughout multiple graduate-level programs. The results of thoughtful inter-professional education show encouraging development of an interdisciplinary-ready workforce.

\section{Implications for Counselors and Counselor Education Programs}

Counselors are missing from palliative care and interdisciplinary teams. Although they have the skills and knowledge to provide end-of-life care, few if any counselors are considered to be members of palliative care interdisciplinary teams. Scant research has been conducted to determine how well counselors fit into interdisciplinary palliative care teams.

Many hospitals employ a typical palliative care model, which contains the key members of medicine, nursing, chaplaincy, and social work. Counselors could also be part of it. The ultimate goal of the hospital is to improve the overall quality of care for patients and to support the family. How the hospital accomplishes this goal can vary from one hospital to another.

Opportunity exists within the palliative care field to utilize the strengths of counselors and the counseling profession. However, a counselor-based palliative care 
model does not resemble traditional interdisciplinary palliative care team composition or service delivery. One innovative and creative approach to palliative care, completely delivered by counselors, has been in use for over 10 years at a large tertiary care hospital in the southeastern United States.

This novel approach to hospital palliative care with an expanded role for counselors is named the Transitions and Palliative Care Therapy (TPCT) Model (Babcock \& Robinson, 2011). Using master's prepared counselors, the TPCT model from Babcock and Robinson (2011) delivers "palliative care to hospitalized patients through a patient-centered, interdisciplinary approach, relying on family systems therapy integration into standard medical practice" (p. 491). The program has grown over time and became an accepted standard of practice in the medical center.

With an emphasis on communication, this program was created using family therapy as a foundation. Palliative care physicians and nurses are part of the model, though they are used in much smaller numbers which reduces the cost associated with higher use of physicians and nurses. Counselors make initial contact with the family and enhance communication between the family and medical personnel throughout the patient's hospital stay. This model of palliative care has proved to be an effective method of managing very complex hospital palliation cases (Babcock \& Robinson, 2011) and is an ongoing program (C. Babcock, personal communication, November 10, 2014). A second TPCT model is in current operation a large southeastern city and is successful (C. Babcock, personal communication, October 26, 2015).

Counselors have the potential to be valuable members of traditional interdisciplinary palliative care teams. If counseling programs include education and 
practica on death, dying, and end-of-life care, then future counselors will have the background and preparation for work in this dynamic area of human experience. Planning will be required to introduce and effectively launch new curriculum centered on death and dying within a counselor education program. Perhaps counselor education students could take part in classes from other related disciplines (e.g., social work or psychology) in order to learn about the field.

A lack of knowledge from the both the classroom or experience within a clinical practica could be considered a weakness of counselor education programs, if counselors want to be considered for interdisciplinary palliative care team work. Counselors cannot expect to be part of the palliative care discussion unless they are prepared. Although the psychosocial field is crowded, room is available for counselors in palliative care, and with the number of elderly who will be approaching the end-of-life within the next twenty years, many human helpers will be needed to improve the quality of care for patients and their families. With preparation and training, counselors can make a place for themselves in palliative care.

\section{Conclusion}

On the basis of this study alone, it is difficult to be certain about all the factors that created the effective interdisciplinary palliative care team experiences for the chaplain interns and social work interns. Changes in curricula and new opportunities for interprofessional learning were created and seemed to benefit the interns as they participated on interdisciplinary care teams. Communication and collaboration within the interdisciplinary team occurred and supported effective patient care as stated by the interns. Many of the expected challenges and barriers from the literature were not 
evidenced in the interns' experiences, which suggested local interdisciplinary teams have improved how they work together to deliver palliative care.

The eagerness of the chaplain interns and the social work interns to find employment due to their interest in palliative care as a result of their placement is encouraging. No interns were disheartened by the atmosphere of death and dying; many wrote they were anticipating a future in a palliative care related field (i.e., oncology social work). There is reason to be hopeful for the future of well-trained interdisciplinary palliative care team chaplains and social workers.

The chaplain interns and social work interns who received interprofessional education and also participated in an interdisciplinary palliative care team clinical placement will be available to provide care for those who will require it within the next twenty years. Both groups of interns completed interdisciplinary palliative care team clinical placements described high functioning interdisciplinary team experiences. The encouraging documented remarks from beginning palliative care chaplain interns and social work interns suggested a promising number of workers will be a ready and enthusiastic workforce to deliver end-of-life care.

The value of the current study presents a different look at the interdisciplinary palliative care team experiences of chaplain interns and social work interns. I suggest the educational program and clinical experience of an interdisciplinary care team not only prepared the interns for future interdisciplinary team work, but also demonstrated how to deliver compassionate interdisciplinary palliative team care. With the demand for more end-of-life care to provide for the large group of elderly patients within the next twenty 
years, the need for interdisciplinary palliative care teams that work well together will be essential.

Counselors possess the skills to work in palliative care; however, few do so, because they are not typically part of the traditional palliative care model of service. Also, counselor training programs rarely provide educational experiences in palliative care which may make counselors less attractive to the current system of palliative care. Hospitals may provide palliative care programs in a variety of types and one innovative and tested counselor-led program is available in the southeastern United States.

Expansion into palliative care for counselors may not be the most familiar path, however, it has the potential to become another source of employment for qualified professionals. Counselors may discover the meaningful and thoughtful work that accompanies end-of-life care. The words from a social work intern summarize the heart of palliative care work:

This profession is all about the important things in life, relationships, love, dignity, compassion, fear, bravery, faith and so much more. I know that the day-to-day tasks of this profession aren't always so lovely, but I believe at its essence, it is sacred work. 


\section{REFERENCES}

Abramson, J. S., \& Mizrahi, T. (2003). Understanding collaboration between social workers and physicians: Application of a typology. Social Work in Health Care, 37(2), 71-100. doi:10.1300/J010v37n02_04

American Academy of Pediatrics, Pediatric Palliative Care and Hospice Care Commitments, Guidelines, and Recommendations. (2013). Section on Hospice and Palliative Medicine and Committee on Hospital Care. Pediatrics, 132, 966972. doi:10.1542/peds.2013-2731

Babcock, C. W., \& Robinson, L. E. (2011). A novel approach to hospital palliative care: An expanded role for counselors. Journal of Palliative Medicine, 14(4), 491-500. doi:10.1089/fpm.2010.0432

Bakitas, M., Lyons, K. D., Hegel, M. T., Balan, S., Brokaw, F. C., Seville, J., ...Ahles, T. (2009). Effects of a palliative care intervention on clinical outcomes in patients with advanced cancer: The project ENABLE II randomized controlled trial. The Journal of the American Medical Association, 302(7), 741-749. doi:10.1001/jama.2009.1198

Benton, K., Stephens. J., Vogel, R., Ledlow, G., Ackermann, R., Babcock, C., \& McCook, G. (2015). The influence of race on end-of-life choices following a counselor-based palliative consultation. American Journal of Hospice \& Palliative Care, 32(1), 84-89. doi:10.1177/1049909113506782

Bernat, J. L. (2005). Medical futility definition, determination, and disputes in critical care. Neurocritical Care, 2, 198-205. doi:10.1385

Blacker, S., \& Deveau, C. (2010). Social work and interprofessional collaboration in palliative care. Progress in Palliative Care, 18(4), 237-243. doi:10.1179/096992610X12624290277141

Bokhour, B. G. (2006). Communication in interdisciplinary team meetings: What are we talking about? Journal of Interprofessional Care, 20(4), 349-363. doi:10.1080/13561820600727205.

Bookbinder, M., \& McHugh, M. E. (2010). Symptom management in palliative care and the end-of-life care. Nursing Clinics of North America, 45(3), 271-327. doi:10.1016/j.cnur.2010.04.002 
Breitbart, W., Gibson, C., Poppito, S. R., \& Berg, A. (2004). Psychotherapeutic interventions at the end-of-life: A focus on meaning and spirituality. Canadian Journal of Psychiatry, 49(6), 366-372.

Bronstein, L. R. (2003). A model for interdisciplinary collaboration. Social Work, 48(3), 297-306.

Bruera, E., \& Hui, D. (2010). Integrating supportive and palliative care in the trajectory of cancer: Establishing goals and models of care. Journal of Clinical Oncology, 28(25), 4013-4017. doi:10.1200.JCO.2010.29.5618

Buring, S. M., Bhushan, A., Broeseker, A., Conway, S., Duncan-Hewitt, W., Hansen, L., \& Westberg, S. (2009). Interprofessional education: Definitions, student competencies, and guidelines for implementation. American Journal of Pharmaceutical Education, 73(4). Retrieved from http:/www.ncbi.nllm.nih.gov/pmc/articles/PMC2720355/

Cadge, W., Calle, K., \& Dillinger, J. (2011). What do chaplains contribute to large academic hospitals? The perspectives of pediatric physicians and chaplains. Journal of Religion and Health, 50(2), 300-312. doi:10.1007/s10943-011-9474-8

Chambliss, D. F. (1996). Beyond caring: Hospitals, nurses, and the social organization of ethics. Chicago, IL: The University of Chicago Press.

Choi, B. C. K., \& Pak, A. W. P. (2006). Multidisciplinarity, interdisciplinarity and transdisciplinarity in health research, services, education and policy: 1. Definitions, objectives, and evidence of effectiveness. Clinical and Investigative Medicine, 29(6), 351-364.

Clark, L., Leedy, S., McDonald, L., Muller, B., Lamb, C., Mendez, T., Kim, S., \& Schonwetter, R. (2007). Spirituality and job satisfaction among hospice interdisciplinary team members. Journal of Palliative Medicine, 10(6), 13211328. doi:10.1089/jpm.2007.0035

Colnerud, G. (2013). Brief Report: Ethical problems in research practice. Journal of Empirical Research on Human Research Ethics, 8(4), 37-41. doi:10.1525/jer.2013.8.4.37

Common Standards for Professional Chaplaincy. (2006). Southern Medical Journal, 99(6), 680-681.

Connor, S. R. (2009). Hospice and palliative care: The essential guide. New York, NY: Routledge. 
Connors, Jr., A. F., Dawson, N. V., Desbiens, N. A., Fulkerson, W. J., Goldman, L., Knaus, W. A., ...Ranshohoff, D. (1995). A controlled trial to improve care for seriously ill hospitalized patients: The study to understand prognoses and preferences for outcomes and risks of treatment (SUPPORT). The Journal of the American Medical Association, 274, 1591-1598. doi:10.1001/jama.1995.03530200027032

Council for Accreditation of Counseling and Related Educational Programs Standards (2009). Retrieved from http://www.cacrep.org/wp-content/uploads/2013/12/2009Standards.pdf

Creswell, J. W. (2012). Educational research: Planning, conducting, and evaluating quantitative and qualitative research ( $4^{\text {th }}$ ed.). Boston, MA: Pearson.

Creswell, J. W. (2013). Quantitative Inquiry \& Research Design ( $3^{\text {rd }}$ ed.). Thousand Oaks, CA: Sage.

Cummings, I. (1998). The interdisciplinary team. In D. Doyle, G. W. C. Hanks, \& N. MacDonald (Eds.), Oxford Textbook of Palliative Medicine (pp. 19-30). New York, NY: Oxford University Press.

Daly, B. J., Douglas, S. L., Gunzler, D., \& Lipson, A. R. (2013). Clinical trial of a supportive care team for patients with advanced cancer. Journal of Pain and Symptom Management, 46(6), 775-784.

doi:http://dx.doi.org/10.1016/j.jpainsymman.2012.12.008

Davis, M. P., Walsh, D., LeGrand, S. B., \& Lagman, R. (2002). End-of-life care: The death of palliative medicine? Journal of Palliative Medicine 5(6), 813-814. doi:10.1089/10966210260498989

Dionne-Odom, J. N., Kono, A., Frost, J., Jackson, L., Ellis, D., Ahmed, A., ....Bakitas, M. (2014). Translating and testing the ENABLE: CHF-PC concurrent palliative care model for older adults with heart failure and their family caregivers. Journal of Palliative Care, 17(9), 995-1004. doi:10.1089/jpm.2013.0680

Doka, K. J. (2009). Counseling individuals with life-threatening illness. New York, NY: Springer.

Doyle, D., Hanks, G., \& MacDonald, N. (1998). Introduction. In D. Doyle, G. W. C. Hanks, \& N. MacDonald (Eds.), Oxford Textbook of Palliative Medicine (pp. 38). New York, NY: Oxford University Press.

Edwards, A., Pang, N., Shiu, V., \& Chan, C. (2010). The understanding of spirituality and the potential role of spiritual care in end-of-life and palliative care: A metastudy of qualitative research. Palliative Medicine, 24 (8), 753-770.

doi: $10.1177 / 0269216310375860$ 
Etherington, K. (2007). Ethical research in reflexive relationships. Qualitative Inquiry, 13(5), 599-616. doi:10.1177.1077800407301175

Federal interagency forum on age-related statistics. 2012 older Americans: Key indicators of well-being. Retrieved from http://www.agingstats.gov/agingstatsdotnet/main_site/default.aspx

Ferrell, B. R., Smith, T. J., Levit, L., \& Balogh, E. (2014). Improving the quality of cancer care: Implications for palliative care. Journal of Palliative Medicine, 17(4), 393-399. doi:10.1089/jpm.2013.0536

Feudtner, C. (2007). Collaborative communication in pediatric palliative care: A foundation for problem-solving and decision-making. Retrieved from http://www.ncbi.nlm.nih.gov/pmc/articles/PMC2151773/

Fewster-Thuente, L., \& Velsor-Friedrich, B. (2008). Interdisciplinary collaboration for Healthcare professionals. Nursing Administration Quarterly, 32(1), 40-48. doi:10.1097/01.NAQ0000305946.31193.6

Fife, R. B. (2002). Are existential questions the norm for terminally ill patients? Journal of Palliative Medicine, 5(6), 815-817. doi:10.1089/10966210260498998

Finestone, A. J., \& Inderwies, G. (2008). Death and dying in the US: The barriers and the benefits of palliative and hospice care. Clinical Interventions in Aging, 3(3), 595599. Retrieved from www.ncbi.nlm.nih.gov.pmc/articles/PMC2682390

Finley, I., Higginson, I. J., Goodwin, D. M., Cook, A. M., Edwards, A. G. K., Hood, K., Douglas, H-R., \& Norman, C. E. (2002). Palliative care in hospital, hospice, at home: Results from a systematic review. Annals of Oncology, 13(S4), 257-264. doi:10.1093/annonc/mdf668

Fiorelli, J. S. (1988). Power in work groups: Team member's perspectives. Human Relations, 41(1), 1-12. doi:10.1177/001872678804100101

Fitchett, G., Lyndes, K. A., Cadge, W., Berlinger, N., Flanagan, E., \& Misasi, J. (2011). The role of professional chaplains on pediatric palliative care teams: Perspectives from physicians and chaplains. Journal of Palliative Medicine, 14(6), 704-707. doi:10.1089/jpm.2010.0523

Flannelly, K. J., Flannelly, K. J., Emanuel, L. L., Handzo, G. F., Galek, K., Silton, N. R., \& Carlson, M. (2012). A national study of chaplaincy services and end-of-life outcomes. BioMed Central, 11(10). doi:10.1186/1472-684X-11-10.

Ford, T., \& Tartaglia, A. (2006). The development, status, and future of healthcare chaplaincy. Southern Medical Journal, 99(6), 675-679.

doi:10.1097/01.smj.0000220893.37354.1e

Frankl, V. E. (2006). Man's search for meaning. Boston, MA: Beacon Press. 
Gachoud, D., Albert, M., Kuper, A., Stroud, L., \& Reeves, S. (2012). Meanings and perceptions of patient-centeredness in social work, nursing and medicine: A comparative study. Journal of Interprofessional Care, 26, 484-490. doi:10.3109/13561820.2012.717553

Gade, G., Venohr, I., Conner, D., McGrady, K., Beane, J., Richardson, R. H., ...Della Penta, R. (2008). Impact of an inpatient palliative care team: A randomized controlled trial. Journal of Palliative Medicine, 11(2), 180-190. doi:10.1089/jpm.2007.0055

Gelfman, L. P., Meier, D. E., \& Morrison, R. S. (2008). Does palliative care improve quality? A survey of bereaved family members. Journal of Pain and Symptom Management, 36(1), 22-28. doi:10.1016/j.jpainsymman.2007.09.0

Glesne, C. (2011). Becoming qualitative researchers: An introduction $\left(4^{\text {th }}\right.$ ed.). Boston, MA: Allyn \& Bacon.

Goldsmith, J., Wittenberg-Lyles, E., Rodriguez, D., \& Sanchez-Reilly, S. (2010). Interdisciplinary geriatric and palliative care team narratives: Collaboration practices and barriers. Qualitative Health Research, 20, 93-104. doi: $10.1177 / 1049732309355287$

Guillemin, M., \& Gillam, L. (2004). Ethics, reflexivity, and ethically important moments in research. Qualitative Inquiry, 10(2), 261-280. doi:10.1177/1077800403262360

Handzo, G. F., Flannelly, K. J., Kudler, T., Fogg, S. L., Harding, S. R., Hasan, Y. H..., \& Taylor, B. E. (2008a). "What do chaplains really do" II. Interventions in the New York chaplaincy study. Journal of Health Care Chaplaincy, 14(1), 39-56. doi:10.1080/08854720802053853

Harper, III, J. M., \& Rudnick, J. E. (2010). The role of the chaplain in palliative care. In G. Hanks, N. I. Cherny, N. A. Christakis, M. Fallon, S. Kaasa, \& R. K. Portenoy, (Eds.), Oxford Textbook of Palliative Medicine ( $4^{\text {th }}$ ed.), (pp.197-205). New York, NY: Oxford University Press.

Hartrick-Doane, G., Stajduhar, K., Causton, E., Bidgood, D., \& Cox, A. (2012). End-oflife care and interprofessional communication: Not simply a matter of "more." Health and Interprofessional Practice, 1(3), eP1028, 1-14. Retrieved from http://www.dx.doi.org/10.7772/2159-1253.1028

Haugen, D. F., Nauck, F., \& Caraceni, A. (2010). The interdisciplinary team. In G. Hanks, N. I. Cherny, N. A. Christakis, M. Fallon, S. Kaasa, \& R. K. Portenoy, (Eds.), Oxford Textbook of Palliative Medicine ( $4^{\text {th }}$ ed.), (pp.167-176). New York, NY: Oxford University Press. 
Hays, R. M., Valentine, J., Haynes, G., Geyer, J. R., Villareale, N., McKinstry, B., Varni, J. W., \& Churchill, S.S. (2006). The Seattle pediatric palliative care project: Effects on family satisfaction and health-related quality of life. Journal of Palliative Medicine, 9(3), 716-728. doi:10.1089/jpm.2006.9.716

Head, B. A., Earnshaw, L. A., Greenberg, R. B., Morehead, R. C., Pfeifer, M. P., \& Shaw, M. A. (2012). "I will never forget": What we learned from medical student reflections on a palliative care experience. Journal of Palliative Medicine, 15(5), 535-541. doi:10.1089/jpm.2011.0391

Head, B. A., Schapmire, T., Hermann, C., Earnshaw, L., Faul, A., Jones, C., ...Pfeifer, M. (2014). The Interdisciplinary Curriculum for Oncology Palliative Care Education (iCOPE): Meeting the challenge of interprofessional education. Journal of Palliative Medicine, 17(10), 1107-1114. doi:10.1089/jpm.2014.0070

Hearn, J., \& Higginson, I. J. (1998). Do specialist palliative care teams improve outcomes for cancer patients? A systematic literature review. Palliative Medicine, 12, 317 332. doi:10.1191/026921698676226729

Henneman, E. A., Lee, J. L., \& Cohen, J. I. (1995). Collaboration: A concept analysis. Journal of Advanced Nursing, 21,103-109. doi:10.1046/j.13652648.1995.21010103.x

Higginson, I. J., \& Evans, C. J. (2010). What is the evidence that palliative care teams Improve outcomes for cancer patients and their families? The Cancer Journal, 16(5), 423-435. doi:10.1097/PPO.0b013e3181f684e5

Higginson, I. J., Finlay, I., Goodwin, D. M., Cook, A. M., Hood, K., Edwards, A. G. K., Douglas, H-R., \& Norman, C. E. (2002). Do hospital-based palliative teams improve care for patients or families at the end-of-life? Journal of Pain and Symptom Management, 23(2), 96-106. Retrieved from http://www.jpsmjournal.com/article/S0885-3924(01)00406-7/pdf

Howe, K. R., \& Dougherty, K. C. (1993). Ethics, institutional review boards, and the changing face of educational research. Educational Researcher, 22 (9), 16-21. Retrieved from http://www.colorado.edu/UCB/AcademicAffairs/education/faculty/kennethhowe/ Docs/Howe_Dougherty_Ethics_IRBs_Changing_Face_of_Ed_Research.pdf

Hurley, S. L., Barg, F. K., Strumpf, N., \& Ersek, M. (2014). Same agency, different teams: Perspectives from home and inpatient hospice care. Qualitative Health Research. Advance online publication. doi:10.1177/1049732314554091

Interprofessional Education Collaborative Expert Panel. (2011). Core competencies for interprofessional collaborative practice: Report of an expert panel. Washington, D.C.: Interprofessional Education Collaborative. Retrieved from http://www.aacn.nche.edu/education-resources/ipecreport.pdf 
Institute of Medicine. (2008). Cancer Care for the Whole Patient: Meeting Psychosocial Health Needs. Washington, DC: The National Academies Press. Retrieved from http://nap.edu/catalog/11993.html

Institute of Medicine. (2014). Dying in America: Improving quality and honoring individual preferences near the end-of-life. Washington, DC: The National Academies Press. Retrieved from http://www.iom.edu/Reports/2014/Dying-InAmerica-Improving-Quality-and- Honoring-Individual-Preferences-Near-theEnd-of-life.aspx

Johnson, R. B. (1997). Examining the validity structure of qualitative research. Education, 118(2), 282-292.

Junger, S., Pestinger, M., Elsner, F., Krumm, N., \& Radbruch, L. (2007). Criteria for successful multiprofessional cooperation in palliative care teams. Palliative Medicine, 21, 347-354. doi:10.1177/0269216307078505

Klarare, A., Hagelin, C. L., Furst, C. J., \& Fossum, B. (2013). Team Interactions in specialized palliative care teams: a qualitative study. Journal of Palliative Medicine, 16(9), 1062-1069. doi:10.1089/jpm.2012.0622.

Leclerc, B-S., Blanchard, L., Cantinotti, M., Couturier, Y., Gervais, D., Lessard, S., \& Mongeau, S. (2014). The effectiveness of interdisciplinary teams in end-of-life palliative care: A systematic review of comparative studies. Journal of Palliative Care, 30(1), 44-54. Retrieved from http://www.researchgate.net/publication/262337977_The_effectiveness_of_interd isciplinary_teams_in_end-oflife_palliative_care_A_systematic_review_of_comparative_studies

Leung, K-K., Tsai, J-S., Cheng, S-Y., Liu, W-J., Chiu, T-Y., Wu, C-H., \& Chen, C-Y. (2010). Can a good death and quality of life be achieved for patients with terminal cancer on a palliative care unit? Journal of Palliative Medicine, 13(12), 14331438. doi:10.1089/jpm.2010.0240

Lynch, M., Dahlin, C., Hultman, T., \& Coakley, E. E. (2011). Palliative care nursing, defining the discipline? Journal of Hospice \& Palliative Nursing, 13(2), 106-111. doi:10.1097/NHJ.0b013e3182075b6e

Maples, M. F., \& Abney, P. C. (2006). Baby boomers mature and gerontological counseling comes of age. Journal of Counseling \& Development, 84, 3-9. doi:10.1002/j.1556-6678.2006.tb00374.x

May, R., \& Yalom, I. (1995). Existential psychotherapy. In R. J. Corsini \& D. Wedding (Eds.), Current Psychotherapies, $5^{\text {th }}$ ed. (pp. 262-292). Itasca, IL: F. E. Peacock Publishers. 
Mazor, K. M., Roblin, D. W., Greene, S. M., Lemay, C. A., Firneno, C. L., Calvi, J., Prouty, C. D., Horner, K., \& Gallagher, T. H. (2012). Toward patient-centered cancer care: Patient perceptions of problematic events, impact, and response. Journal of Clinical Oncology, 30(15), 1784-1790. doi:10.1200/jco.2011.38.1384

McCallin, A. (2001). Interdisciplinary practice-A matter of teamwork: An integrated literature review. Journal of Clinical Nursing, 10(4), 419-428. doi:10.1046/j.1365-2702.2001.00495.x

Meier, D. E. (2011). Increased access to palliative care and hospice services: Opportunities to improve value in health care. The Milbank Quarterly, 89(3), 343380. doi: 10.111/j.1468-0009.2011.00632.x

Meier, D. E., \& Beresford, L. (2008). The palliative care team. Journal of Palliative Medicine, 11(5), 677-681. doi:10.1089/jpm.2008.9907

Meier, D. E., \& Brawley, O. W. (2011). Palliative care and the quality of life. Journal of Clinical Oncology, 29(20), 2750-2752. doi:10.1200/JCO.2011.35.9729

Mellor, M. J., Hyer, K., \& Howe, J. L. (2002). The geriatric interdisciplinary team approach: Challenges and opportunities in educating trainees together from a variety of disciplines. Educational Gerontology, 28, 867-880. doi:10.1080/03601270290099868

Miles, M. B., Huberman, A. M., \& Saldana, J. (2014). Qualitative data analysis: A methods sourcebook. Thousand Oaks, CA: Sage.

Millspaugh, D. (2005). Assessment and response to spiritual pain: Part II. Journal of Palliative Medicine, 8(6), 1110-1117. doi:10.1089/jpm.2005.8.1110.

Mitchell, D. (2011). Spirituality and cultural issues at the end-of-life. Medicine, 39 (11), 678-679. doi: http://dx.doi.org/10.1016/j.mpmed.2007.11.011

Monroe, B. (2010). Social work in palliative medicine. In G. Hanks, N. I. Cherny, N. A. Christakis, M. Fallon, S. Kaasa, \& R. K. Portenoy, (Eds.), Oxford Textbook of Palliative Medicine ( $4^{\text {th }}$ ed.), (pp.184-196). New York, NY: Oxford University Press.

Mor, V., \& Birnbaum, H. (1983). Medicare legislation for hospice care: Implications of the National Hospice Study. Health Affairs, 2(2), 80-90. doi:10.1377/hlthaff.2.2.80

Mor, V., \& Kidder, D. (1985). Cost savings in hospice: Final results of the National Hospice Study. Health Services Research, 20(4), 407-422.

Morrow, S. L. (2005). Quality and trustworthiness in qualitative research in counseling psychology. Journal of Counseling Psychology, 52(2), 250-260. doi:10.1037/0022-0167.52.2.250 
Mount, B. M. (1976). The problem of caring for the dying in a general hospital; the palliative care unit as a possible solution. Canadian Medical Association Journal, 115, 119-121. Retrieved from http://www.ncbi.nlm.nih.gov/pmc/articles/PMC1878558/pdf/canmedaj014840031.pdf

Moustakas, C. E. (1994). Phenomenological research methods. Thousand Oaks, CA: Sage.

Nancarrow, S. A., Booth, A., Ariss, S., Smith, T., Enderby, P., \& Roots, A. (2013). Ten principles of good interdisciplinary team work. Human Resource Health, 11(19). doi:10.1186/1478-4491-11-19.

National Association of Social Workers. NASW standards of practice for palliative \& end-of-life care. Washington, DC: NASW Press, 2004. Retrieved from http://www.socialworkers.org/practice/bereavement/standards/standards0504New

National Association of Social Workers. Indicators for the Achievement of the NASW standards for Cultural Competence in Social Work Practice. Washington, DC: NASW Press, 2007. Retrieved from https://www.socialworkers.org/practice/standards/Indicators_for_Cultural_Compe tence.asp

National Hospice and Palliative Care Organization. (2012). NHPCO facts and figures: Hospice care in America (2012 Edition). Retrieved from http://www.nhpco.org

National Consensus Project for Quality Palliative Care. Clinical practice guidelines for quality palliative care, second edition. Pittsburgh, PA: National Consensus Project for Quality Palliative Care, 2009. Retrieved from http://www.nationalconsensusproject.org/

National Consensus Project for Quality Palliative Care. Clinical practice guidelines for quality palliative care, third edition. Pittsburgh, PA: National Consensus Project for Quality Palliative Care, 2013. Retrieved from http://www.nationalconsensusproject.org/

Nelson, J. E., Cortez, T. B., Curtis, J. R., Lustbader, D. R., Mosenthal, A. C., Mulkerin, C., ...Puntillo, K. A. (2011). Integrating palliative care in the ICU. Journal of Hospice \& Palliative Nursing, 13(2), 89-94. doi:10.1097/NJH.0b013e318203d9ff

Nelson, J. E., Puntillo, K. A., Pronovost, P. J., Walker, A. S., McAdam, J. L., Iloa, D., \& Penrod, J. (2010). In their own words: Patients and families define high-quality palliative care in the intensive care unit. Critical Care Medicine, 38(3), 808-818.

O'Connor, M., \& Fisher, C. (2011). Exploring the dynamics of interdisciplinary palliative care teams in providing psychosocial care: "Everybody thinks that everybody can do it and they can't." Journal of Palliative Medicine, 14(2), 191-196. doi:10.1089/jpm.2010.0229 
O'Connor, M., Fisher, C., \& Guilfoyle, A. (2006). Interdisciplinary teams in palliative care: A critical reflection. International Journal of Palliative Nursing, 12, 132135. doi:http://dx.doi.org/10.12968/ijpn.2006.12.3.20698

Ogleby, M., \& Goldstein, R. D. (2014). Interdisciplinary care-using your team. The Pediatric Clinics of North America, 61, 823-834. doi:10.1016/j.pc1.2014.04.009

Orbach, I. (2008). Existentialism and suicide. In A. Tomer, G. T. Eliason, \& P. T. P. Wong (Eds.), Existential and spiritual issues in death attitudes (pp. 7-37). New York, NY: Lawrence Erlbaum.

Parker-Oliver, D., Bronstein, L. R., \& Kurzejeski, L. (2005). Examining variables related to successful collaboration on the hospice team. Health \& Social Work, 30(4), 279-286. doi:10.1093/hsw/30.4.279

Patton, M. Q. (2015). Qualitative research \& evaluation methods $\left(4^{\text {th }}\right.$ ed.). Thousand Oaks, CA: Sage.

Peteet, J. R., \& Balboni, M. J. (2013). Spirituality and religion in oncology. CA: A Cancer Journal for Clinicians, 63, 280-289. doi:10.1002/caac.21187

Peters, L., \& Sellick, K. (2006). Quality of life of cancer patients receiving inpatient and home-based palliative care. Journal of Advanced Nursing, 53(5), 524-533. doi:10.1111/j.1365-2648.2006.03754.x

Pfeifer, M., Hermann, C., Holthouser, A., Shaw, A., Head, B., Schapmire, T., Faul, A., \& Woggin, F. (2012). The Development of an Interdisciplinary Oncology Palliative Care Curriculum, The National Cancer Institute and the University of Louisville Department of Medicine.

Ponte, P. R., Gross, A. H., Milliman-Richard, Y. J., \& Lacey K. (2010). Interdisciplinary teamwork and collaboration: An essential element of a positive practice environment. In A. Debisette \& J. Vessey (Eds.), Annual Review of Nursing Research, 28, (pp.1159-1189). New York, NY: Springer Publishing. doi: http://dx.doi.org/10.1891/0739-6686.28.159

Puchalski, C. M. (2007). Spirituality and the care of patients at the end-of-life: An essential component of care. Omega, 56(1), 33-36.

Puchalski, C. M. (2010). Religion, medicine and spirituality: What we know, what we don't know and what we do. Asia Pacific Journal of Cancer Prevention, 11, MECC Supplement, 45-49.

Puchalski, C. M. (2012). Spirituality in the cancer trajectory. Annals of Oncology, 23(supp3), 49-55. doi:10.1093/annonc/mds088. 
Puchalski, C. M., Lunsford, B., Harris, M. H., \& Miller, T. (2006). Interdisciplinary spiritual care for seriously ill and dying patients: A collaborative model. The Cancer Journal, 12(5), 398-416.

Quill, T. E. (2001). Caring for patients at the end-of-life. New York, NY: Oxford University Press.

Quill, T. E., \& Abernethy, A. P. (2013). Generalist plus specialist palliative care creating a more sustainable model. The New England Journal of Medicine, 368, 11731175. doi:10.1056/NEJMp1215620

Remke, S. S., \& Schermer, M. M. (2012). Team collaboration in pediatric palliative care. Journal of Social Work in End-of-life \& Palliative Care, 8, 286-296. doi:10.1080/1552425.2012.735551

Rome, R. B., Luninais, H. H., Bourgois, D. A., \& Blais, C. M. (2011). The role of palliative care at the end-of-life. The Oschner Journal, 11(4), 348-352.

Saldana, J. (2009). The coding manual for qualitative researchers. Thousand Oaks, CA: Sage.

Sargeant, J., Loney, E., \& Murphy, G. (2008). Effective interprofessional teams: “Contact is not enough" to build a team. Journal of Continuing Education in the Healthcare Professions, 28(4), 228-234. doi:10.1002/chp.189

Saunders, C. (1978). Hospice care. The American Journal of Medicine, 65, 726-728.

Saunders, C. (1998). Forward. In D. Doyle, G. W. C. Hanks, \& N. MacDonald (Eds.), Oxford Textbook of Palliative Medicine (pp. v-ix). New York, NY: Oxford University Press.

Saunders, C. (2000). The evolution of palliative care. Patient Education and Counseling, 4l(1), 7-13. doi:10.1016/S0738-3991(00)00110-5

Schutz, A. (1970). On phenomenology and social relations (H. R. Wagner, Ed.). Chicago, IL: University of Chicago Press.

Sherman, D. W. (2010). Nursing and palliative care. In G. Hanks, N. I. Cherny, N. A. Christakis, M. Fallon, S. Kaasa, \& R. K. Portenoy, (Eds.), Oxford Textbook of Palliative Medicine (4 ${ }^{\text {th }}$ ed.), (pp. 177-183). New York, NY: Oxford University Press.

Sinclair, S., Raffin, S., Pereira, J., \& Guebert, N. (2006). Collective soul: The spirituality of an interdisciplinary palliative care team. Palliative and Supportive Care 4, 1324. doi:10.1017/S147895150606032 
Sinclair, S. (2011). Impact of death and dying on the personal lives and practices of palliative and hospice care professionals. Canadian Medical Association Journal, 183(2), 180-187. doi:10.1503/cmaj.100511

Smith, T. J., Temin, S., Alesi, E. R., Abernethy, A. P., Balboni, T. A., Basch, E. M., ...Von Roenn, J. H. (2012b). American Society of Clinical Oncology provisional clinical opinion: The integration of palliative care into standard oncology care. Journal of Clinical Oncology, 30(8), 880-887. doi:10.1200/JCO.2011.38.5161

Strang, S., \& Strang, P. (2002). Questions posed to hospital chaplains by palliative care patients. Journal of Palliative Medicine, 5(6), 857-864. doi:10.1089/10966210260499041

Taylor, Jr., D. H., Ostermann, J., Van Houtven, C. H., Tulsky, J. A., \& Steinhauser, K. (2007). What length of hospice use maximizes reduction in medical expenditures near death in the US Medicare program? Social Science \& Medicine, 65, 14661478. doi:10.1016/j.socscimed.2007.05.28

Teno, J. M., Clarridge, B. R., Casey, V., Welch, L. C., Wetle, T., Shield, R., \& Mor, V. (2004). Family perspectives on end-of-life care as the last place of care. The Journal of the American Medical Association, 291(1), 88-93. doi:10.1001./jama.291.1.88

Tomer, A., \& Eliason, G. (2005). Life regrets and death attitudes in college students. OMEGA, 51(3), 173-195. doi:10.2190/B22C-CFFA-216G-R2PN

Tomer, A., \& Eliason, G. T. (2008). Existentialism and death attitudes. In A. Tomer, G. Eliason, \& P.T.P. Wong (Eds.), Existential and Spiritual Issues in Death Attitudes (pp. 7-37). New York, NY: Lawrence Erlbaum.

U.S. Department of Health and Human Services, Centers for Disease Control and Prevention National Center for Health Statistics National Vital Statistics System. (2013). Deaths: Final data for 2010 (Report No. 61). Retrieved from http://www.cdc.gov/nchs/data/nvsr/nvsr61/nvsr61_04.pdf

U.S. Department of Health and Human Services, Centers for Medicare \& Medicaid Services, Medicare and Medicaid Programs: Hospice Conditions of Participation; final rule. (2008). Retrieved from www.gpo.gov/fdsys/pkg/FR-2008-0605/pdf/08-1305.pdf

Valle, R. (1998). Phenomenological inquiry in psychology. New York, NY: Plenum Press.

Van Manen, M. (1990). Researching lived experience. Ontario, CA: The University of Western Ontario.

Van Manen, M. (2014). Phenomenology of practice. Walnut Creek, CA: Left Coast Press. 
Vissers, K. C. P., van den Brand, M. W. M., Jacobs, J., Groot, M., Veldhoven, C., Verhagen, C., Hasselhaar, J., \& Engels, Y. (2012). Palliative medicine update: A multidisciplinary approach. Pain Practice, 13(7), 576-588. doi:10.1111/papr.12025

Webster, J., \& Kristjanson, L. J. (2002). Long-term palliative care workers: More than a story of endurance. Journal of Palliative Medicine, 5(6), 865-875. doi:10.1089/10966210260499050

Wesley, C., Tunney, K., \& Duncan, E. (2004). Educational needs of hospice social workers: Spiritual assessment and interventions with diverse populations. American Journal of Hospice \& Palliative Medicine, 21(1), 40-46.

Wiecha, J., \& Pollard, T. (2004). The interdisciplinary eHealth team: Chronic care for the future. Journal of Medical Internet Research, 6(3), e22. doi:10.2196/jmir.6.3.e22

Wiles, R., Crow, G., Heath, S., \& Charles, V. (2008). The management of confidentiality and anonymity in social research. International Journal of Social Research Methodology, 11(5), 417-428. doi:10.1080/13645570701622231

Wilson, D. C., Ajemian, I., \& Mount, B. M. (1978). The Royal Victoria Hospital palliative care service. Death Education, 2(1-2), 3-19.

Wittenberg-Lyles, E., Oliver, D. P., Demiris, G., Baldwin, P., \& Regehr, K. (2008). Communication dynamics in hospice teams: Understanding the role of the chaplain in interdisciplinary team collaboration. Journal of Palliative Medicine, 11(10), 1330-1335. doi:10.1089/jmp.2008.0165

Wittenberg-Lyles, E., Oliver, D. P., Demiris, G., Baldwin, P., \& Regehr, K. (2009). Exploring interpersonal communication in hospice interdisciplinary team meetings. Journal of Gerontological Nursing, 35(7), 38-45. doi:10.3928/00989134-20090527-04

Wittenberg-Lyles, E., Oliver, D. P., Demiris, G., \& Regehr, K. (2010). Interdisciplinary collaboration in hospice team meetings. Journal of Interprofessional Care, 24(3), 264-273. doi:10.3109/13561820903163421.

World Health Organization. (2003). WHO definition of palliative care. Retrieved from http://www.who.int/cancer/palliative/definition/en/

World Health Organization. (2010). Framework for action on interprofessional education and collaborative practice. Department of Human Resources for Health; Geneva, Switzerland. Retrieved from http://www.who.int/hrh/resources/framework_action/en/index.html

World Health Organization. (n.d.) Team building. Retrieved from www.who.int/camcer/modules/Team\%20building.pdf 
Wu, H-L., \& Volker, D. L. (2012). Humanistic nursing theory: Application to hospice and palliative care. Journal of Advanced Nursing, 68(2), 471-479. doi:10.1111/j.1365-2648.2011.05770.x

Xyrichis, A., \& Lowton, K. (2008). What fosters or prevents interprofessional teamworking in primary and community care? A literature review. International Journal of Nursing Studies, 45, 140-153. doi:10.1016/j.ijnurstu.2007.01.015

Yalom, I. (1980). Existential psychotherapy. New York, NY: Basic Books.

Youngwerth, J., \& Twaddle, M. (2011). Cultures of interdisciplinary teams: How to foster good dynamics. Journal of Palliative Medicine, 14(5), 650-654. doi:10.1089/jpm.2010.0395

Youngwerth, J., Brackett, H., Wenger, B., Bortolotto, S., Baiamonte, V., Petri, J., Marso, J., \& Sande, M. (2015). Compassionate Extubation in a Non-Intensive Care Unit Setting [abstract]. Journal of Hospital Medicine, 10 (supplement 2). Abstract retrieved from http://www.shmabstracts.com/abstract/compassionate-extubationin-a-non-intensive-care-unit-setting/

Zimmerman, C., Riechelmann, R., Krzyzanowska, M., Rodin, G., \& Tannock, I. (2008). Effectiveness of specialized palliative care: A systematic review. Journal of the American Medical Association, 299(14), 1698-1709. doi:10.1001/jama.299.14.1698

Zwarenstein, M., Goldman, J., \& Reeves, S. (2009). Interprofessional collaboration: Effects of practice based interventions on professional practice and healthcare outcomes. Cochrane Database of Systematic Reviews, 3, Art. No.:CD000072. doi:10.1002/14651858.CD000072.pub2. 
APPENDICES 
Appendix A

\section{iCOPE Critical Reflection Writing and Group Experience}

For this assignment, you are to focus on one patient that you observed during your palliative care clinical experience or from the video (Letting Go, Wit, etc.) that you viewed. Address the areas described below. Your critical reflection paper should not exceed 3-4 pages in length (double-spaced, 12 font, please). Use the template (included) that has been created for this exercise. Include the three sections below.

1. In 1-2 paragraphs, summarize the clinical scenario in language that non-clinical readers could understand.

- Briefly describe the patient and his/her situation, diagnosis and prognosis, brief history of the disease, and how/why the patient entered palliativefocused care.

- Describe the predominant palliative care needs of this patient and his/her family. Think holistically about their medical, psychosocial and spiritual needs.

- Briefly describe how well these needs are currently being met. What team members are involved and what interventions are occurring? How do you judge the progress being made towards the goals for this patient's care?

2. Now-put on your critical thinking cap. Address the following critical review questions.

- How could the patient's/family care be enhanced?

- Was there an adequate interdisciplinary assessment and care plan?

- Describe the communication among the parties involved (team communication: with each other, the patient and family, and other providers)

- Discuss how this interdisciplinary and patient/family oriented setting supported quality palliative care. If it didn't, how did it fall short?

3. Lastly, but most importantly, reflect personally on what you learned from your involvement with this patient. This should be the longest portion of your paper.

- How did the experience touch you personally? Professionally?

- How did the experience impact your future practice as a healthcare provider?

- Describe observations that had a positive or negative impact on you.

- How has your perception of palliative care changed based on the experience? 
Your reflection paper should be submitted to the iCOPE Program Coordinator, Carol Jones at carol.jones@louisville.edu. For chaplain \& social work students submit at least one week prior to your interdisciplinary critical reflection writing group session. For nursing students refer to the due date given by your instructor. You should also bring a copy of your paper to the discussion group you are assigned to attend as part of the iCOPE curriculum. At that time, you will share your reflections with other students and professionals. This will provide an opportunity to both debrief about your experience and learn from the experiences of others. Do not complete Evaluation of Clinical Rotation \& Critical Reflection until AFTER the group session. 
Appendix B

\section{Subject Informed Consent Document}

\section{Interdisciplinary Curriculum for Oncology Palliative Care Education}

Sponsor assigned \# 1R25CA148005

Industry contract \# OICB091401

Sponsor-NCI address:

National Cancer Institute

National Institutes of Health

6120 Executive Blvd.

Rockville, MD 20852

Investigator (s) name \& address: Mark Pfeifer, MD

MDR Building, Ste. 110

511 South Floyd Street

Louisville, KY 40202

Site (s) where study is to be conducted: University of Louisville Schools of Medicine, Nursing and Social Work

Phone numbers for subjects to call for questions: 502 562-4014

\section{Introduction and Background Information}

You are invited to participate in a research study because your academic program requires you to complete a new interdisciplinary palliative care curriculum. The study is being conducted by Mark Pfeifer, MD. The study is sponsored by the National Cancer Institute and the University of Louisville, Department of Medicine. The study will take place at the University of Louisville's Schools of Medicine, Nursing and Social Work. Approximately 150 subjects will be invited to participate.

\section{Purpose}

The purpose of this study is to evaluate the impact of a novel interdisciplinary curriculum being piloted at the University of Louisville. Your will be completing this curriculum and the evaluation instruments as part of your required coursework.

\section{Procedures}

You will be expected to complete the iCOPE curriculum consisting of didactic online modules, a clinical rotation, and two small group interdisciplinary activities of the course of one semester. As part of the iCOPE curriculum, you will also be expected to complete a series of instruments evaluating the overall experience and its impact on your knowledge, attitudes and skills. The instruments you will be asked to complete will require approximately 2 hours. The investigators are requesting permission to access and analyze all curriculum evaluation instruments that will be completed as part of the delivery of the required curriculum. 
Additionally, the researchers will analyze the evaluations of your work by the iCOPE faculty members.

These evaluations will assess whether you have met the expectations of the learning activities.

As part of the curriculum, you will be asked to write a critical reflection paper. The researchers plan to analyze these papers in aggregate using qualitative data analysis software.

If you consent to participate in the study, all of the above described data will be analyzed and use to contribute to generalizable knowledge via publications and presentations. However, no personal identifying information will be used. Signing this consent document indicates that you agree to allow the researchers to use the information you provide.

\section{Potential Risks}

There are no foreseeable risks outside of the discomfort you might experience answering survey questions. There may be unforeseen risks.

\section{Benefits}

The possible benefits of this study include participation in this novel curriculum and your learning related to the knowledge, skills and values of interdisciplinary palliative care. The evaluation information collected may not benefit you directly, but it will be used by faculty to evaluate, improve and revise the curriculum for future students. Therefore, the information learned in this study may be helpful to others.

\section{Compensation}

You will not be compensated for your time, inconvenience, or expenses while you are in this study.

\section{Confidentiality}

Total privacy cannot be guaranteed. Your privacy will be protected to the extent permitted by law. If the results from this study are published, your name will not be made public. While unlikely, the following may look at the study records:

The sponsor and companies hired by the sponsor to oversee the study

The University of Louisville Institutional Review Board, Human Subjects

Protection Program Office

Office for Human Research Protections (OHRP)

Your responses will be recorded and maintained on a password protected computer.

Pictures of you participating in curriculum activities may be taken for use in disseminating information about iCOPE.

I permit my picture to be used in reports and presentations about iCOPE.

\section{Signature}


I do not permit my picture to be used in reports and presentations about iCOPE.

\section{Signature}

\section{Conflict of Interest}

This study involves a conflict of interest because the institution and investigator will be compensated for our participation in it. Please ask the investigator how the institution and investigator will benefit by your participation in the study.

\section{Voluntary Participation}

Taking part in this study is voluntary. You may choose not to take part at all. If you decide to be in this study you may stop taking part at any time. If you decide not to be in this study or if you stop taking part at any time, you will not lose any benefits for which you may qualify.

\section{Research Subject's Rights, Questions, Concerns, and Complaints}

If you have any concerns or complaints about the study or the study staff, you have three options:

You may contact the principal investigator at 502 562-4014.

If you have any questions about your rights as a study subject, questions, concerns or complaints, you may call the Human Subjects Protection Program Office (HSPPO) 502 852-5188. You may discuss any questions about your rights as a subject, in secret, with a member of the Institutional Review Board (IRB) or the HSPPO staff. The IRB is an independent committee composed of members of the University community not connected with these institutions. The IRB has reviewed this study.

If you want to speak to a person outside the University, you may call 1-877-8521167. You will be given the chance to talk about any questions, concerns or complaints in secret. This is a 24 hour hotline answered by people who do not work at the University of Louisville.

This informed consent document is not a contract. This document tells you what will happen during the study if you choose to take part. Your signature indicates that this study has been explained to you, that your questions have been answered, and that you agree to take part in the study. You are not giving up any legal rights by signing this informed consent document. Your will be given a copy of this consent form to keep for your records.

Printed Name of Subject/

Legal Representative
Signature of Subject/

Legal Representative
Date Signed 
Printed Name of Person

Explaining Consent Form
Signature of Person Explaining

Consent Form (if other than the Investigator)
Printed Name of Investigator

LIST OF INVESTIGATORS

Mark Pfeifer, MD

Carla Hermann, $\mathrm{PhD}$

Amy Holthouser, MD

Ann Shaw, MD

Barbara Head, $\mathrm{PhD}$

Tara Schapmire, $\mathrm{PhD}$

Anna Faul, PhD

Frank Woggin, $\mathrm{PhD}, \mathrm{BBC}$
Signature of Investigator

PHONE NUMBERS

562-4014

$852-8397$

$852-1597$

$852-7944$

$852-3014$

$852-3738$

852-1981

217-5146
Date Signed

Date Signed 


\section{Appendix C}

\section{IRB Outcome Letters}

\section{UNIEERSIIY OOF \\ Human Subjects Protection Program Office \\ MedCenter One - Suite 200 \\ 501 E. Broadway \\ Louisville, KY 40202-1798 \\ Office: 502.852 .5188 Fax: 502.852 .2164}

$\begin{array}{ll}\text { DATE: } & \text { July 16, } 2015 \\ \text { TO: } & \text { Lisa M Hooper } \\ \text { FROM: } & \text { The University of Louisville Institutional Review Board } \\ \text { IRB\#: } & 15.0631 \\ \text { STUDY TITLE: } & \begin{array}{l}\text { INTERDISCIPLINARY PALLIATIVE CARE TEAMS: INSIGHTS AND EXPERIENCES } \\ \text { OF CHAPLAIN INTERNS AND SOCIAL WORK INTERNS }\end{array} \\ \text { REFERENCE \#: } & 370683 \\ \text { DATE OF REVIEW: } & 07 / 15 / 2015 \\ \text { IRB STAFF CONTACT: } & \begin{array}{l}\text { Name: Jacqueline S. Powell } \\ \text { Phone: } 852-4101\end{array} \\ & \text { Email: jspowe01@Louisville.edu }\end{array}$

This study was reviewed on $07 / 15 / 2015$ and determined by the Chair of the Institutional Review Board that the study is exempt according to 45 CFR 46.101(b) under category 1: Instructional strategies in established educational settings.

This study was also approved through 45 CFR 46.116 (D), which means that it has been granted a waiver of informed consent because it meets the following criteria:

- The research involves no more than minimal risk to the subjects.

-The waiver or alteration will not adversely affect the rights and welfare of the subjects.

- The research could not practicably be carried out without the waiver or alteration.

-Whenever appropriate, the subjects will be provided with the additional pertinent information after participation.

Documents/Attachments reviewed and approved:

Submission Components

\begin{tabular}{|l|l|l|l|}
\hline IRB Protocol & Version 1.2 & $06 / 30 / 2015$ & Approved \\
\hline
\end{tabular}

Please be advised that any study documents submitted with this protocol should be used in the form in which they were approved.

Since this study has been approved under the exempt category indicated above, no additional reporting, such as submission of Progress Reports for continuation reviews, is needed. If your research focus or activities change, please submit an Amendment to the IRB for review to ensure that the indicated exempt category still applies. Best wishes for a successful study. Please send all inquiries to our office email address at hsppofc@louisville.edu

Thank you for your submission.

Sincerely,

Pate $M$ Lusach 
DATE:

October 15, 2015

TO:

Lisa M Hooper

FROM:

The University of Louisville Institutional Review Board

IRB NUMBER:

15.0631

STUDY TITLE:

INTERDISCIPLINARY PALLIATIVE CARE TEAMS: INSIGHTS AND EXPERIENCES

REFERENCE \#: OF CHAPLAIN INTERNS AND SOCIAL WORK INTERNS

429501

DATE OF REVIEW:

$10 / 14 / 2015$

IRB STAFF CONTACT: Name: Jacqueline S. Powell

Phone: 852-4101

Email: jspowe01@Louisville.edu

The following item(s) have been received by the Human Subjects Protection Program Office and were reviewed by the Chair/Vice-Chair of the Institutional Review Board (IRB):

\section{Submission Components}

Submission Form

\begin{tabular}{|l|l|}
\hline Form Name & Outcome \\
\hline
\end{tabular}

\begin{tabular}{|l|l|}
\hline IRB Amendment Form & Exempt \\
\hline
\end{tabular}

The modifications include:

- The study inclusion criteria has changed to include documents (the data are pre-existing text) from Fall, 2012 thru Spring, 2015

- The previously approved IRB Protocol was approved to include documents (the data are preexisting text) from Spring 2014 thru Spring 2015

This information has been reviewed and determined by the Chair of the Institutional Review Board (IRB) not to change the exempt status of this study.

If you have any questions, please contact the HSPPO at (502) 852-5188 or hsppofc@louisville.edu 
Appendix D

Chaplain Intern Attribute Codes

\begin{tabular}{|c|c|c|c|}
\hline Reflection & Age & Gender & Setting \\
\hline 1 & 25 & Female & $\mathrm{N} / \mathrm{A}$ \\
\hline 2 & 31 & Female & Norton Hospital \\
\hline 3 & 60 & Female & Norton Hospital \\
\hline 4 & 29 & Male & Audubon Hospital \\
\hline 5 & 31 & Male & Norton Hospital \\
\hline 6 & 37 & Male & Baptist Health Louisville \\
\hline 7 & 41 & Male & Norton Hospital \\
\hline 8 & 58 & Male & Norton Hospital \\
\hline 9 & 25 & Female & $\mathrm{N} / \mathrm{A}$ \\
\hline 10 & 33 & Female & University of Louisville Hospital \\
\hline 11 & 29 & Female & University of Louisville Hospital \\
\hline 12 & 51 & Female & University of Louisville Hospital \\
\hline 13 & 60 & Female & Norton Hospital \\
\hline 14 & $\mathrm{~N} / \mathrm{A}$ & Female & Baptist Health Louisville \\
\hline 15 & 29 & Male & Norton Hospital \\
\hline 16 & 37 & Male & Baptist Health Louisville \\
\hline 17 & 54 & Male & Norton Hospital \\
\hline 18 & $\mathrm{~N} / \mathrm{A}$ & Male & University of Louisville Hospital \\
\hline 19 & 53 & Female & Baptist Health Louisville \\
\hline 20 & 40 & Male & Norton Hospital \\
\hline 21 & 58 & Male & Norton Hospital \\
\hline 22 & 26 & Male & Norton Hospital \\
\hline 23 & 28 & Female & Baptist Health Louisville \\
\hline 24 & 49 & Female & Norton Hospital \\
\hline
\end{tabular}


Appendix E

Social Work Intern Attribute Codes

\begin{tabular}{|c|c|c|c|}
\hline Reflection & Age & Gender & Setting \\
\hline 1 & 29 & Female & Hosparus Inpatient Care Center \\
\hline 2 & 24 & Female & Hosparus Inpatient Care Center \\
\hline 3 & 60 & Female & University of Louisville Hospital \\
\hline 4 & 60 & Female & Norton Hospital \\
\hline 5 & 25 & Female & Baptist Health Louisville \\
\hline 6 & 23 & Female & $\begin{array}{l}\text { Louisville Veterans Administration } \\
\text { Medical Center }\end{array}$ \\
\hline 7 & 26 & Female & Baptist Health Louisville \\
\hline 8 & N/A & Male & $\begin{array}{l}\text { Louisville Veterans Administration } \\
\text { Medical Center }\end{array}$ \\
\hline 9 & 24 & Female & Norton Hospital \\
\hline 10 & 24 & Female & University of Louisville Hospital \\
\hline 11 & 24 & Female & Baptist Health Louisville \\
\hline 12 & 24 & Female & Norton Hospital \\
\hline 13 & 29 & Female & Hosparus Inpatient Care Clinic \\
\hline 14 & 23 & Female & Gilda's Club/Hosparus \\
\hline 15 & 46 & Female & Hosparus Home Visits \\
\hline 16 & 23 & Female & Baptist Health Louisville \\
\hline 17 & 24 & Female & Hospital inpatient to Hosparus \\
\hline 18 & 24 & Female & Hosparus Inpatient Care Clinic \\
\hline 19 & 24 & Female & Baptist Health Louisville \\
\hline 20 & $\mathrm{~N} / \mathrm{A}$ & Male & University of Louisville Hospital \\
\hline 21 & 23 & Female & Baptist Health Louisville \\
\hline 22 & 24 & Female & $\begin{array}{l}\text { Consultants in Blood Disorders and } \\
\text { Cancer }\end{array}$ \\
\hline 23 & 40 & Female & University of Louisville Hospital \\
\hline
\end{tabular}




\section{CURRICULUM VITA}

Ann Hayes Ronald

Home Address: 43 Mockingbird Valley Drive

Louisville, KY 40207

(502) 262-8151

ahayesron@aol.com

Office Address: $\quad$ Square One

6440 Dutchmans Parkway

Louisville, KY 40205

(502) 896-2606

aronald@squareonemd.com

DOB: $\quad$ May 21, 1956

Marital Status: Married, two daughters

Education: $\quad$ Doctor of Philosophy, University of Louisville, Louisville, Kentucky. December 2015. Major: Counselor Education and Supervision

Master of Education, University of Louisville, Louisville, KY. May 2005. Major: Counseling Psychology

Bachelor of Arts, Hanover College, Hanover, IN.

May 1978. Major: Sociology

Internships The Brook Hospital-KMI, January 2010-December 2010

Hosparus, August 2003-May 2004

Kentucky State Board of Psychology

Licensed Psychological Associate

\#KY-0789 (November 2005 to Present)

\section{Kentucky Board of Licensed Professional Counselors}

Licensed Professional Counseling Associate

\#KY-0275 (October 2005 to Present) 


\section{Professional Societies}

American Psychological Association

Kentucky Psychological Association

American Counseling Association

\section{Community Board}

Kilgore Samaritan Counseling Center, 2007-2009

Secretary $(2008,2009)$

\section{Employment}

Square One, Specialists in Child and Adolescent Development (September 2005 to present)

I perform psychological, psychoeducational, and developmental assessments for children, under the supervision of David L. Causey, Ph. D. I am also part of a multidisciplinary team (e. g., medicine, psychology, and speech pathology) which evaluates young children who are suspected of being on the autism spectrum. I write psychoeducational reports and participate in feedback sessions for parents of patients. I also conduct brief developmental assessments and write reports for all incoming preKindergarten and Kindergarten applicants for a local independent school.

Hosparus, formerly Hospice of Louisville (May 2004 to September 2005)

I conducted bereavement counseling for adults, in the home or at the Hosparus' offices. In addition to bereavement counseling, my duties included leading time-limited grief psychoeducational groups and monthly adult grief support groups. The support group locations were: Okolona, Shively, Shelbyville, and Louisville. While at Hosparus, I participated in Families In Transition (F.I.T.) classes, which are court mandated divorce education for parents and children under the age of 18 .

\section{Articles}

Ronald, A. (2015). “Ready, Set, Go!” Kindergarten Readiness, Today’s Family, Winter 2015-2016. 